\title{
Helium II Heat Transfer in LHC Magnets \\ Polyimide Cable Insulation
}

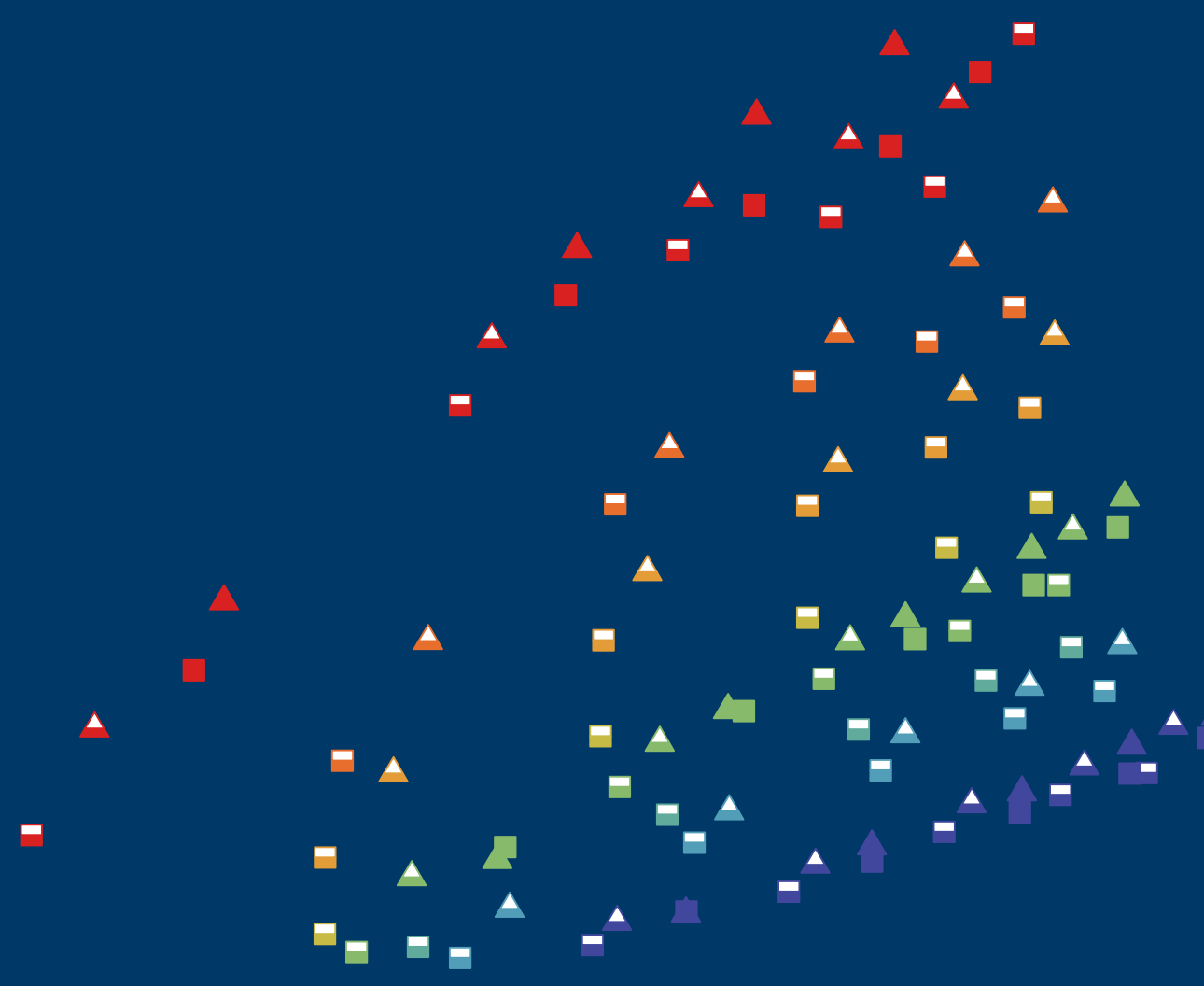

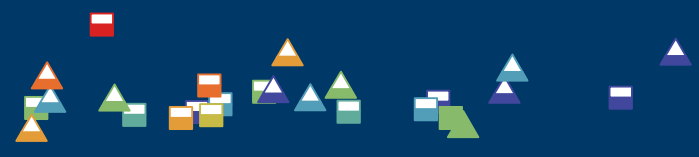

Tiemo Winkler 


\title{
Helium II Heat Transfer in LHC Magnets

\author{
Polyimide Cable Insulation
}

\author{
Tiemo Winkler
}




\section{Graduation committee:}

Chairman Prof. dr. ir J.W.M. Hilgenkamp

Supervisor Prof. dr. ir. H.J.M. ter Brake

Prof. dr. ir. H.H.J. ten Kate

Co-supervisor Dr. R. van Weelderen

Members Dr. B. Baudouy

Dr. M. Breschi

Dr. M.M.J. Dhallé

Prof. dr. ing. B. van Eijk

Dr. rer. nat. T. Köttig

Prof. dr. A.T.A.M. de Waele

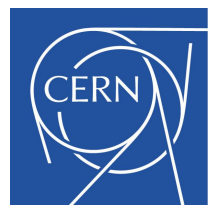

The research described in this thesis was funded by and carried out at:

CERN, European Organization for Nuclear Research, Geneva, Switzerland

Technology Department (TE)

Cryogenics Group (CRG)

Cryolab \& Instrumentation Section (CI)

Helium II Heat Transfer in LHC Magnets

T. Winkler

PhD thesis, University of Twente, The Netherlands

ISBN 978-90-365-4353-8

DOI 10.3990/1.9789036543538

URL https://dx.doi.org/10.3990/1.9789036543538

Printed by Ipskamp Printing, Enschede, The Netherlands

Cover by T. Winkler

(C) T. Winkler, Enschede, 2017 


\title{
HELIUM II HEAT TRANSFER IN LHC MAGNETS
}

POLYIMIDE CABLE INSULATION

\section{DISSERTATION}

\author{
to obtain \\ the degree of doctor at the University of Twente, \\ on the authority of the rector magnificus, \\ prof. dr. T.T.M. Palstra, \\ on account of the decision o the graduation committee, \\ to be publicly defended \\ on Friday, $9^{\text {th }}$ of June 2017 at 12.45 hours
}

by

\section{Tiemo Winkler}

born on the $5^{\text {th }}$ of July 1986

in Münster (Westf.), Germany 
This thesis has been approved by the supervisors:

Prof. dr. ir. H.J.M. ter Brake

Prof. dr. ir. H.H.J. ten Kate 
Quis leget haec?

Flaccus, Satirist, (34-62AD) 



\section{Contents}

\begin{tabular}{lll}
\hline 1 & Introduction & 1
\end{tabular}

2 Heat Transfer in Superconducting Magnets 3

$2.1 \quad$ Superconductivity and its application in the LHC . . . . . . . . . . 3

$2.2 \quad$ Heat Deposition During the Operation of the LHC . . . . . . . . . 7

2.3 Helium as a Technical Cooling Fluid . . . . . . . . . . . . . . . . . . 7

2.3 .1 Helium II Two-Fluid Model . . . . . . . . . . . . . . . . . . 9

2.3 .2 Heat Transfer in Helium II . . . . . . . . . . . . . . . . . 16

2.4 Application of Helium Heat Transfer to Accelerator Magnet Cooling 17

$2.5 \quad$ Thesis Objectives and Outline . . . . . . . . . . . . . 20

3 Experimental Set-up 23

3.1 Measurement Procedure . . . . . . . . . . . . . . . . . . . 23

3.2 Experimental Set-up . . . . . . . . . . . . . . . . . . . . . . . . . . . . . . . . . . . .

3.2 .1 Sample . . . . . . . . . . . . . . . . . . . . . . . . . . . . . . . . . . 24

3.2 .2 Sample Assembly . . . . . . . . . . . . . . . . . . . . . . . . . . . . . . . . . . 27

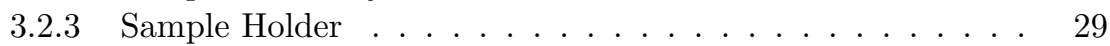

3.2 .4 Pressurised He II Vessel . . . . . . . . . . . . . . . . . . . . . . . . . . . . . . . . 30

3.2 .5 AC Magnetic Field Sample Heating System . . . . . . . . . 31

3.3 Measurement Accuracy . . . . . . . . . . . . . . . . . . 38

3.3 .1 Absolute He Bath Temperature . . . . . . . . . . . . . . . . 38

3.3 .2 He Bath Temperature Stability . . . . . . . . . . . . . . . . 39

3.3 .3 Absolute Sample Temperature . . . . . . . . . . . . . . . . 39

3.3.4 Relative Sample Temperature . . . . . . . . . . . . . . . . . . . 40

3.4 Data Analysis . . . . . . . . . . . . . . . . 40

4 Calorimetry 43

4.1 Adiabatic Method . . . . . . . . . . . . . . . . . . . 43

4.1 .1 Measurement Principle. . . . . . . . . . . . . . . . . . 43

4.1 .2 Accuracy Estimation . . . . . . . . . . . . . . . . . . . . . . . . . . . . . . . . . . . . . 4

4.2 Heat Meter Calorimetry . . . . . . . . . . . . . . . . . . . . . . . . . . . 45

$4.2 .1 \quad$ Measurement Principle. . . . . . . . . . . . . . . . . . . . 45

4.2 .2 Heat Meter Calorimetry Error Discussion . . . . . . . . . . 46

4.3 Calorimetry Results . . . . . . . . . . . . . . . . . . . . . 47

4.3 .1 Adiabatic Method Results . . . . . . . . . . . . . . . . . . 47

4.3 .2 Heat Meter Calorimetry Results . . . . . . . . . . . . . . . . . 48

4.3 .3 Calorimetry Summary . . . . . . . . . . . . . . . 50 
5 Heat Transfer Model 53

5.1 Direct Transition Model for the Effective Thermal Conductivity. . 53

$5.1 .1 \quad$ Critical Velocity $\ldots \ldots \ldots \ldots \ldots$

5.1 .2 Model Limitations . . . . . . . . . . . . . . . . . . 57

5.2 Model Description . . . . . . . . . . . . . . . . . . . . . 58

$5.2 .1 \quad$ Model Composition . . . . . . . . . . . . . . . . 59

5.2 .2 Model Summary _. . . . . . . . . . . . . . . . . . . 66

$5.2 .3 \quad$ Fitting Process $\ldots \ldots \ldots \ldots$. . . . . . . . . . 67

$\begin{array}{llll}6 & \text { Results } & \mathbf{7 1}\end{array}$

6.1 Steady State Measurement Results _. . . . . . . . . . . . . . . . 71

6.1 .1 Measurement Data Analysis . . . . . . . . . . . . . . . . 71

$6.1 .2 \quad$ Experimental Reproducibility and Accuracy . . . . . . . . . 76

$6.1 .3 \quad$ Model Data Analysis . . . . . . . . . . . . . . . . . . . . . . 78

$6.1 .4 \quad$ Different Experimental Conditions _ . . . . . . . . . . . 80

6.1 .5 Temperature range $1.7 \mathrm{~K}$ to $2.1 \mathrm{~K} . \ldots \ldots$. . . . . . . 83

6.2 Transient Results . . . . . . . . . . . . . . . . . . . . . . 90

$6.2 .1 \quad$ Effective Volumetric Heat Capacity. . . . . . . . . . . . . . 91

6.2.2 Transient Effective Thermal Conductivity . . . . . . . . . . 93

6.2 .3 Concluding Remarks $\ldots \ldots \ldots \ldots$. . . . . . . . . . . . . . 97

$\begin{array}{lll}7 \text { Conclusion } & 99\end{array}$

\begin{tabular}{ll}
\hline A Measurement Data & 103
\end{tabular}

\begin{tabular}{ll}
\hline B Two Channel Model Plots & 107
\end{tabular}

\begin{tabular}{ll}
\hline Bibliography & 111
\end{tabular}

$\begin{array}{ll}\text { Summary } & 117\end{array}$

$\begin{array}{ll}\text { Samenvatting } & 119\end{array}$

\begin{tabular}{ll}
\hline Acknowledgement & 121
\end{tabular} 


\section{Introduction}

The discovery of electro-magnetism and an increased interest in particle physics led to the construction of various accelerators to study the nature of matter. One variety of these accelerators is called a Synchrotron and uses a circular layout, which relies on a large number of electro-magnets to control the beam and only uses very few acceleration elements [1. The Large Hadron Collider (LHC) at the European Organisation for Nuclear Research (CERN) in Geneva, Switzerland, is the latest and most powerful iteration of this accelerator type [1.

The magnets have to create a magnetic field that scales with the size of the machine and with the maximum beam energy. In early iterations the electromagnets used in this accelerator type were made from copper cables with an increasing amount of turns in a magnet for an increasing magnetic field. However, the practical limit for such a magnet is reached at a magnetic field of about $2 \mathrm{~T}$ [2. For higher magnetic field strengths the operation of such a magnet becomes uneconomical and the size of the accelerators unreasonably large due to the low achievable magnetic field strength.

The solution to this is the use of superconductors in the coils of the magnets. A superconductor is a material that has no electric resistance when it is cooled below a material dependent critical temperature. This phenomenon was found by the Dutch physicist H.K. Onnes at the beginning of the $20^{\text {th }}$ century and has driven technology advances since then [3].

The use of such a material to build magnets has two main advantages as compared to copper. First a superconductor can carry a significantly higher transport current for the same cable dimensions, which decreases the size of the magnet while still generating a much stronger magnetic field than a normal conducting electro-magnet. The second advantage of a superconducting magnet is the decreasing operation cost due to the absence of any electrical resistance in the superconductor in direct current operation. Instead, energy is now needed to maintain the low operation temperature of the superconductor. Starting from a certain point it is economically more interesting to operate a superconducting accelerator than a normal conducting accelerator.

In the LHC two particle beams are circulated. One beam circulates clockwise and the second beam circulates counter-clockwise. At certain points the two beams are brought into collision and the resulting particle shower is detected in one of the four main experiments at the LHC. Both beams are not a continuous stream of particles but consist of up to 2808 bunches with each bunch containing $10^{11}$ protons [4. During the operation of the LHC these bunches will repeatedly cross each other and each time a small number of protons will collide, creating other particles in the process. At the same time some protons are scattered during a bunch crossing, an effect that will slowly but continuously increase the beam size. 


\section{Introduction}

To counteract this effect a collimation system is used that limits the maximum size of the beam by absorbing protons that are too far away from the desired beam orbit. During collimation the protons are stopped in the collimator and deposit their energy in the collimator in the process. This energy is then creating new particles in a particle shower that is concentrated behind the collimator where they are absorbed by the superconducting magnets. This is one of the effects that leads to particle showers outside of the experimental regions, which are then in turn absorbed by the superconducting magnet structure behind the collimator.

These absorbed particle showers generate heat inside the magnet, which needs to remain below the current sharing temperature in order to retain superconductivity 4. Since the superconducting magnet winding is very close to the beam pipe it is exposed to the highest heat loads creating the necessity to know and understand the heat transfer between the superconducting magnet winding and the helium bath in order to guarantee a continuous and safe operation of the superconducting magnets.

This work contributes to the understanding of the heat transfer in He II in the confined space of superconducting Rutherford cable polyimide insulation. Measurements are conducted in a wide temperature range, in superfluid Helium in saturated and pressurised condition for different mechanical compression of the Rutherford type cable stack. Information on the transient and steady-state heat transfer is drawn from the measurements. Focus is on the non-stationary thermal behaviour, which is becoming increasingly relevant with the recent increase of the beam energy and luminosity of the LHC [5]. 


\section{Heat Transfer in Superconducting Magnets}

\subsection{Superconductivity and its application in the LHC}

Superconductivity is a phenomenon that appears in certain materials when they are cooled below their critical temperature. Taking a closer look at the conditions when a material is superconducting one finds that it not only depends on the temperature but also on the current density and the applied magnetic field [6]. These critical values are not independent but instead are a function of each other. Together they form a critical surface and depending on the conditions the superconductor is exposed to, it is either in the superconducting state or in the normal conducting state. As an example Figure 2.1 shows the critical surface of the superconductor Niobium-Titantium.

Niobium-Titanium is today's most commonly used superconductor due to its easy fabrication and manipulation guaranteeing a constant good wire quality. It is used for all superconducting magnets in the LHC, where the superconductor is operating at a temperature of $1.9 \mathrm{~K}$ to achieve a magnetic field of $8.33 \mathrm{~T}$. Together with the critical surface this results in a temperature margin of only $1.6 \mathrm{~K}$ before the superconductor becomes normal conducting [4. The temperature of the magnet is chosen to be $1.9 \mathrm{~K}$ for three reasons. Firstly, with a decreasing temperature of the superconductor higher magnetic flux densities can be sustained. Secondly, the critical current density of the superconductor increases with a decrease of the conductor temperature. The third reason is related to the special properties of the coolant helium at this temperature. When the temperature is lowered below a critical value helium passes through a phase transition and it becomes a superfluid where the effective thermal conductivity of helium increases by orders of magnitude [7. This, compared to other materials, extraordinarily high effective thermal conductivity enables the construction of very compact high field magnets as they are used in the LHC.

The biggest magnets that are used in the LHC are the main bending magnets and the main quadrupole magnets, which are responsible for the bending and the focusing of the beam, respectively. In total the LHC uses 1232 main bending magnets and 392 main quadrupole magnets in eight arcs [4]. Figure 2.2 shows a cut of a main bending magnet, where the main components of the magnet are shown. The main bending magnet is a twin aperture dipole magnet where the clockwise beam circulates in one aperture and the anti-clockwise beam in the second aperture. The main bending magnet is thus technically speaking two dipole magnets in one common shell, one dipole magnet for each beam. The magnet winding consists of two layers made from two superconducting cables. The windings 


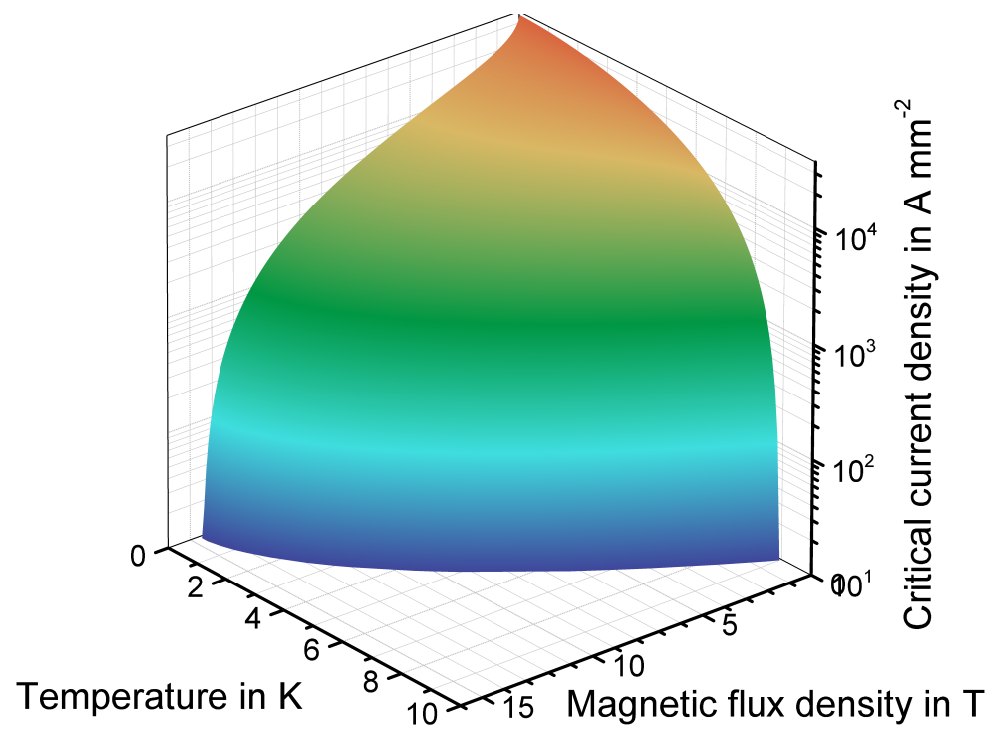

Figure 2.1. The critical surface of the superconductor Niobium-Titanium as calculated by a fit from L. Bottura [8]. If the parameters of the superconducting material are below the surface the superconducting material is in the superconducting state. If the parameters are outside of the critical surface the superconducting material is in the normal conducting state.

are compressed by a collar in order to minimise the movement of the cable and guarantee a homogeneous magnetic field in the cold bore. The magnetic field is concentrated and amplified by an iron yoke. The collar and the iron yoke are not a single piece but are fabricated from $3 \mathrm{~mm}$ and $5.8 \mathrm{~mm}$ thick sheets, respectively with a gap of $0.24 \mathrm{~mm}$ between the sheets in the collar and $0.46 \mathrm{~mm}$ between the sheets in the yoke. The whole assembly is called the magnet cold mass and is placed inside a helium tank. During operation the gaps in the assembly are filled with sub-cooled liquid helium, which provides the cooling for the superconductor. The helium in the helium tank transports the heat from the superconductor to the heat exchanger tube mounted in the top part of the cold mass assembly. The heat exchanger tube is partly filled with liquid helium at saturation pressure and is directly connected to the cooling plant.

Figure 2.3 shows a cross section of the coil windings of a main dipole magnet. The beam tube in the centre and the two layer construction of the magnet are clearly visible. During the operation most of the secondary particle showers will hit the magnet in the horizontal beam axis and the centre windings will be exposed to the highest heat loads [9]. These heat loads have to be extracted by the liquid helium from the cable to the heat exchanger tube.

The coil consists of several turns of a superconducting cable. On the left 


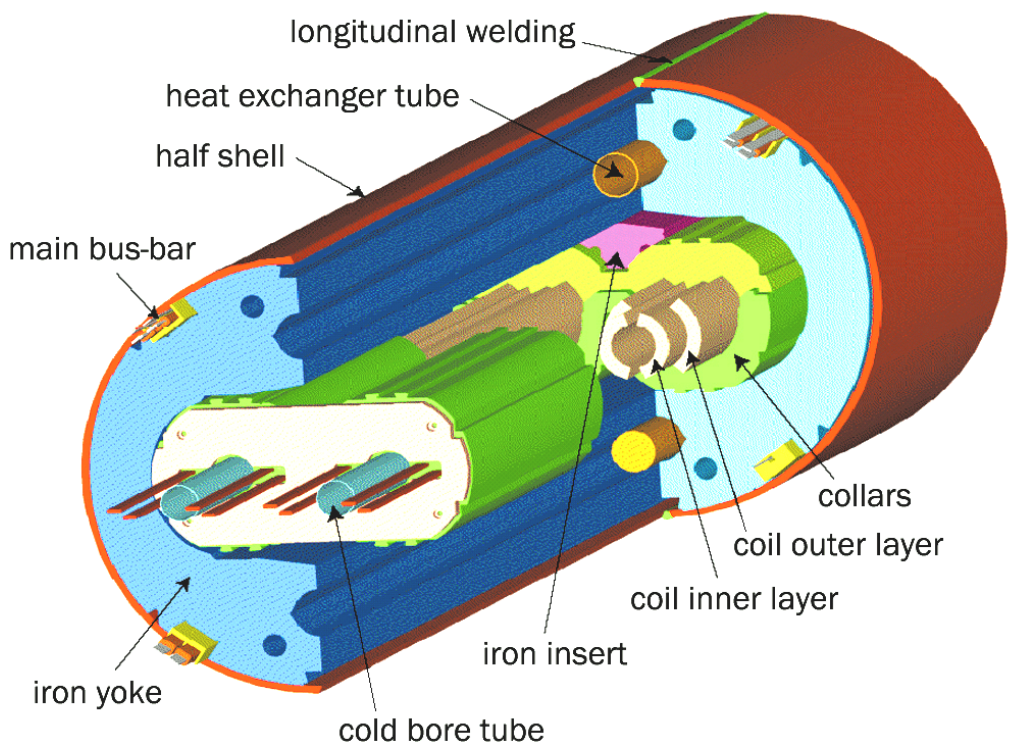

Figure 2.2. Schematics of the cross section of a main bending magnet cold mass of the LHC. This assembly is called the magnet cold mass and is cooled to $1.9 \mathrm{~K}$ in normal operation. The main components of the assembly are labeled. CCERN

of Figure 2.4 the layout of the superconducting cable that is used in the main bending magnets of the LHC is shown. This particular shape of a cable is called a Rutherford cable. It has a rectangular or slightly trapezoidal shape, which facilitates the stacking of cables in race track magnets. The cable used in the main bending magnets inner layers consists of 28 strands with a nominal diameter of $1.065 \mathrm{~mm}$. Each of the 28 strands consists of 8800 superconducting filaments embedded in a copper matrix for electric and thermal stability [10. On the right of Figure 2.4 the cross section of one strand is depicted, where the different filaments are clearly distinguishable from the copper matrix.

The cable itself is wrapped with a dielectric insulation to guarantee a sufficient electrical insulation of the cable. This insulation consists of three individual layers wrapped in a well defined pattern. This pattern is essential for the cooling of the cable since it forms a dense layer around the cable and limits the contact of the helium in the voids of the cable with the helium outside of the coil windings. This insulation is, as will be seen later, a research focus since the beginning of the design phase of the LHC. 


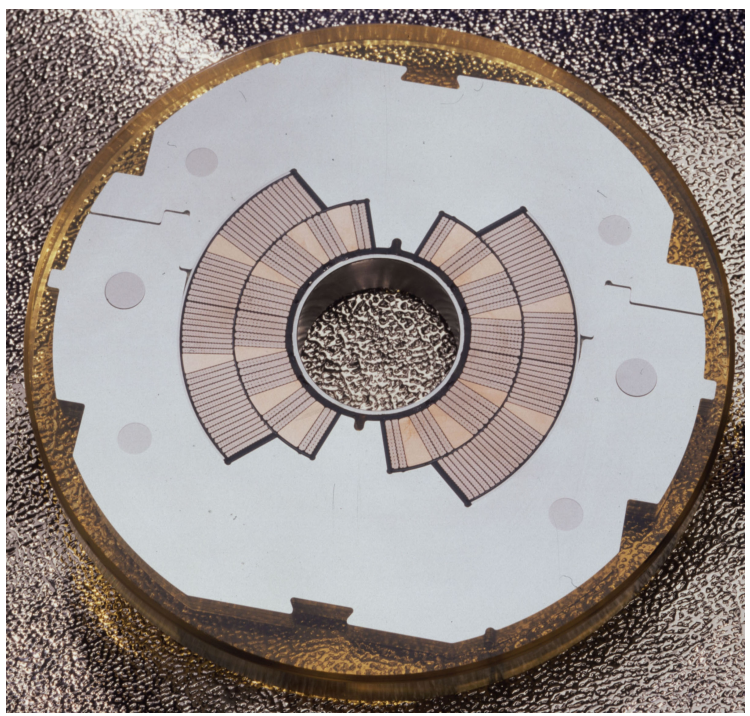

Figure 2.3. Picture of the cross section of a main bending dipole magnet prototype. The beam tube in the centre and the two layer configuration of the coil winding can clearly be identified. The coil windings consist of 6 individual blocks, which are separated by wedges. This layout is necessary for a homogenous field in the beam tube. (C)CERN

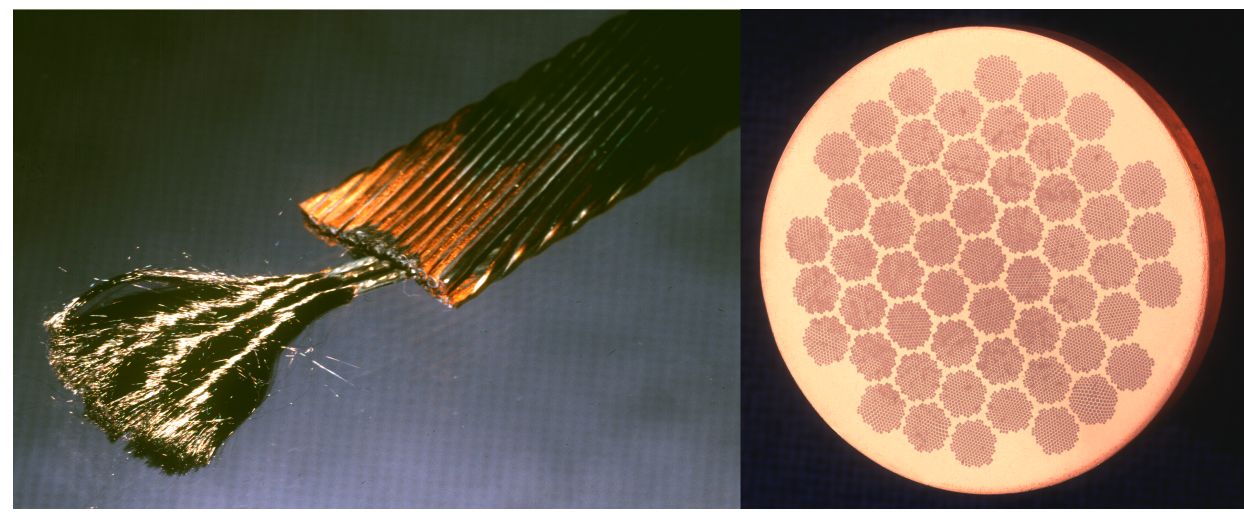

Figure 2.4. Left: Picture of a superconducting Rutherford cable. From two strands the copper matrix has been etched away and the bare superconductor filaments are visible. Right: Cross section of a strand. The hexagonal structure of the superconducting filaments and the copper matrix is clearly visible. CCERN 
Heat that is deposited inside the cable has to be transported from the strands to the helium in the voids and then through the insulation to the pressurised helium bath. From there the heat is transported via the heat exchanger tube to the cooling plant.

\subsection{Heat Deposition During the Operation of the LHC}

Aside from the heat load to the helium vessel from the environment there are also heat loads that result from the operation of the particle accelerator itself 4 . These heat loads are all related to the presence of a beam in the accelerator. Even though these heat loads are minimised by design as far as possible they cannot be completely reduced to zero and need to be dealt with by the cooling system.

During the operation of the LHC heat is generated inside the superconductor that needs to be extracted. The reasons for this heat generation are numerous and have to be split into steady-state heat generation and transient heat generation. This is advised because of the nature of the heat transfer that results from the heat generation. Steady-state heat deposition occurs continuously throughout the operation of the LHC and the heat needs to be extracted continuously. The only material property that influences the temperature rise in the superconductor is the effective thermal conductivity.

On the other hand heat generation that occurs only for a limited duration is influenced by two factors. The effective thermal conductivity and equally important the heat capacity of the cable and the helium, which acts as a temporary thermal buffer.

This means that the steady-state energy deposition that can be tolerated is much smaller than the energy deposition that can be tolerated for short durations. The difference between the two arises from the non-stationary effects in He II heat transfer and the available heat capacities.

Figure 2.5 shows a plot of the calculated quench threshold before a quench in the magnet coil occurs as a function of the pulse duration for four different beam energies. It shows that the tolerable heat deposition in the superconductor is much higher for short duration compared to longer pulse durations. The quench threshold reaches a steady-state value for heat pulse durations longer than $\approx 20 \mathrm{~s}$.

\subsection{Helium as a Technical Cooling Fluid}

The cooling medium used in the LHC is liquid helium. The cold mass of the LHC is built as a double bath cryostat where the magnet part is filled with sub-cooled liquid helium, which is separated from the saturated helium by the heat exchanger pipe mounted in the cold mass [4].

At the end of the 19th century until the twenties of the 20th century different laboratories were rushing to liquify the last so called permanent gases [12]. These permanent gases could not be liquified by just increasing the pressure, these gases 


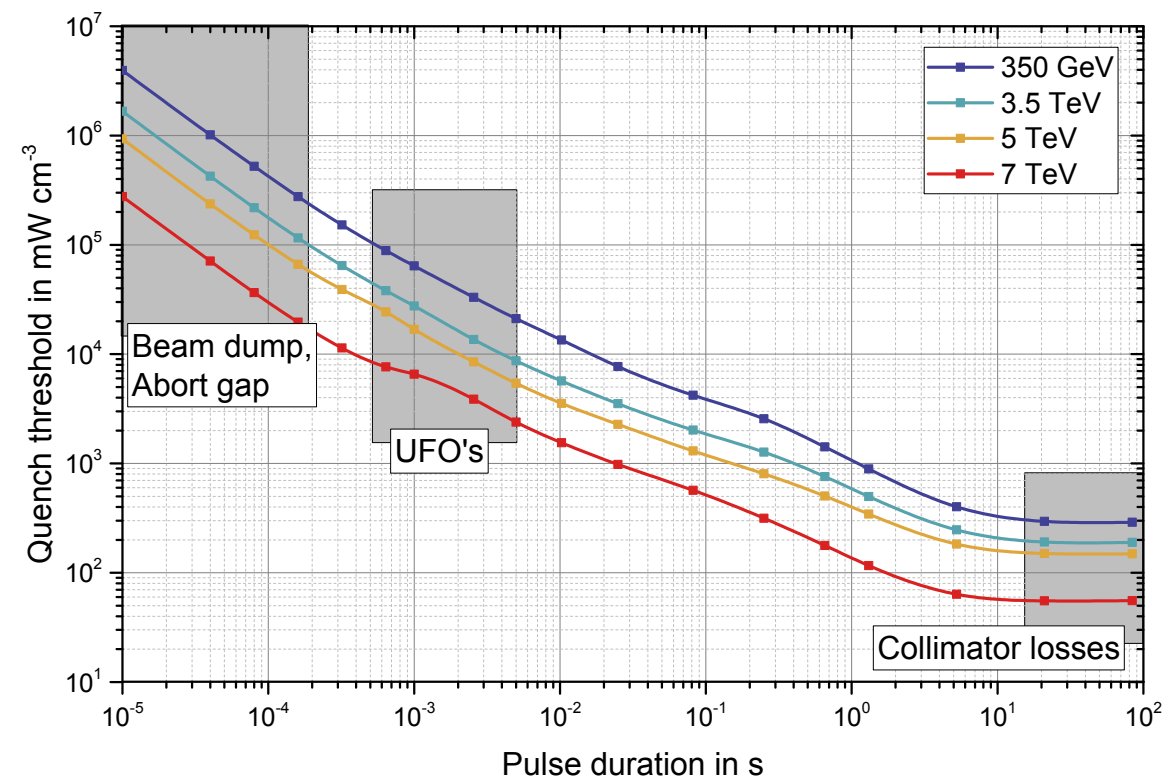

Figure 2.5. Simulation results for the quench threshold in the midplane of a main bending magnet as a function of the pulse duration and beam energy. The grey boxes indicate expected heat loads for different loss sources. The shown data is adapted from [1].

could only be obtained in their liquid state when the temperature of the gas was decreased. The last of the permanent gases to be liquified was helium and H.K. Onnes at the University of Leiden finally announced the liquefaction of helium in 1908 [13].

Almost 30 years later P. Kapitsa 14 and independently J.F. Allen and D. Misener [15] discovered in 1937 that helium has two distinct liquid phases with very different properties. The helium I phase is readily obtained when helium is liquified at $4.2 \mathrm{~K}$ at a pressure of $0.1 \mathrm{MPa}$. When the temperature of the liquid is lowered below $2.1768 \mathrm{~K}$ a new phase, the helium II phase, occurs at a saturation pressure of $5021 \mathrm{~Pa}$. This point is also known as lambda point due to the characteristic shape of the liquid heat capacity in the vicinity of $2.1768 \mathrm{~K}$ [16, 17]. The phase transition between the two helium phases is a second order phase transition since the specific entropy is continuous and the specific heat capacity has a logarithmic singularity [18]. There is also an upper lambda point at a temperature of $1.762 \mathrm{~K}$ and a pressure of 3.0119 MPa. Above this pressure helium is a solid and the line connecting the two lambda points is called lambda line.

Helium II shows very distinct properties such as a viscosity that is dependent on the measurement principle. The viscosity values found can either be zero or have a finite value [7, 19]. It also has an extremely high effective thermal conductivity, 
which soon after its discovery became a focus point of low temperature physics [20]. Experiments showed that the apparent thermal conductivity is six orders of magnitude higher than the thermal conductivity of helium I making it a very interesting research topic. Soon after the discovery of the helium II phase theories were developed to describe these characteristics [21, 22, 23.

\subsubsection{Helium II Two-Fluid Model}

The generally accepted phenomenological theory of helium II is called the two-fluid model and was developed by L. Landau [21] in the year 1941 based on ideas of L. Tisza [22. L. Landau was awarded the physics Nobel prize for his work on the theory of superfluidity in 1962. In his model L. Landau assumes that helium II is composed of two inseparable and completely mixed components. One component he called normal fluid and the second component is named superfluid. R. Feynman then refined Landaus work and formulated the two-fluid model as it is known today 23 .

Each of the two components in He II is associated with specific properties. The normal fluid component is designated as the sole carrier of entropy in the model, while the superfluid component has a specific entropy of zero. The viscosity that is measured in experiments is also only associated with the normal fluid component, the superfluid component having zero viscosity.

The ratio between the normal fluid component and the superfluid component is a function of temperature. Above the lambda line helium consists entirely of normal fluid component. At the lambda temperature $T_{\lambda}$ the superfluid component occurs and the composition of He II shifts with decreasing temperature to higher superfluid component contents [7]:

$$
\frac{\rho_{n}}{\rho}=\left(\frac{T}{T_{\lambda}}\right)^{5.6} \quad \text { for } \quad T \leq T_{\lambda} .
$$

The total density $\rho$ is then the sum of the density of the superfluid component $\rho_{s}$ and the density of the normal fluid component $\rho_{n}$ [7]:

$$
\rho=\rho_{s}+\rho_{n} .
$$

The zero viscosity of the superfluid component was deduced from experiments conducted with porous plugs impenetrable by He I. It was found that helium is flowing through such a porous plug, effectively emptying a reservoir, when the temperature was lowered below the lambda line temperature. It was deduced that this is only possible if the superfluid component has zero viscosity.

Other experiments found that if a heater was switched on in a small He II filled reservoir, separated from the main bath by a porous plug but connected such that they experienced the same vapour pressure, the liquid pressure inside the small reservoir increased. For a small enough temperature increase the pressure increase was found to be linearly dependent on the temperature difference between the reservoir and the bath. This effect is also known as fountain effect. The relation between the pressure increase and the temperature increase can be deduced from 
the specific Gibbs potential by assuming steady state conditions and equal Gibbs potential between the reservoir and the bath. Then the following relation is obtained [7:

$$
\Delta p=\rho s \Delta T,
$$

where $\Delta p$ and $\Delta T$ are the pressure difference and the temperature difference between the reservoir and the bath, respectively. The specific entropy $s$ of He II is equal to the specific entropy of the normal fluid component.

The fountain effect and the fact that the superfluid component can flow unrestricted through porous plugs can be expressed mathematically following [7] as:

$$
\nabla \times v_{s}=0
$$

with $v_{s}$ as the superfluid velocity. This indicates that the superfluid component can not sustain any shear forces and thus does not experience any viscous drag at the wall.

\section{Zero-Net Mass Flow in He II}

In Landau's theory He II is composed of two different components with very different properties and it is necessary to take the conservation of momentum into account. The total momentum of helium II is the sum of the momenta of the two components as follows [7]:

$$
\rho v=\rho_{n} v_{n}+\rho_{s} v_{s} .
$$

Here $v$ denotes the velocity. In the absence of an imposed flow this equation leads to the interesting situation of zero total momentum but non-zero momenta for the two components. This specific situation is called zero net mass flow or internal convection since no mass flow is detected in such systems. Internal convection is responsible for the high effective thermal conductivity, since heat is not transported by conduction but by a convection style mechanism.

Only the normal fluid component can carry entropy and thus a heat flux is always associated with a normal fluid component mass flow from the heat source to the cold sink and a superfluid component mass flow towards the heat source in order to fulfil the zero net mass flow condition. With the known transported heat flux by the normal fluid component the normal fluid velocity can then be calculated following [7] as:

$$
v_{n}=\frac{\dot{q}}{\rho s T},
$$

with $\dot{q}$ the heat flux density and $T$ the temperature. By using the zero net mass flow condition also the superfluid velocity can be determined. 


\section{Laminar Regime}

In experiments with narrow capillaries whose dimensions are large enough for the normal fluid component not to be immobilised due to viscous drag from the wall, the viscosity has been measured under Poiseuille flow conditions [14, 15, 19, 24. Since only the normal fluid component is associated with a viscosity, the measured viscosity is identical to the normal fluid viscosity.

In his formulation of the two fluid model Landau assumes zero viscosity for the superfluid component. Consequently the two equations of motion are formulated in [7] as follows:

$$
\rho_{s} \frac{\delta v_{s}}{\delta t}=\rho_{s} s \nabla T-\frac{\rho_{s}}{\rho} \nabla p,
$$

for the superfluid component with no viscous interaction taken into account and

$$
\rho_{n} \frac{\delta v_{n}}{\delta t}=-\rho_{s} s \nabla T-\frac{\rho_{n}}{\rho} \nabla p+\mu_{n} \nabla^{2} v_{n},
$$

for the normal fluid component where a viscous friction term is added on the right hand side of the equation where $\mu_{n}$ is the normal fluid viscosity.

In order to calculate the heat flux density transported by He II one can combine the equations of motion and obtain a relation between the pressure derivative and the second derivative of the normal fluid velocity:

$$
\nabla p=\mu_{n} \nabla^{2} v_{n}
$$

For known channel dimensions and using the relation for the fountain pressure Equation (2.3) together with the relation between the heat flux density and the normal fluid velocity it is possible to derive the heat flux density as a function of the temperature gradient:

$$
\dot{q}=-\frac{d^{2}(\rho s)^{2} T}{\beta \mu_{n}} \frac{d T}{d x},
$$

where $\beta$ is a dimensionless geometry dependent factor, which is 32 for circular channels and 12 for wide aspect ratio channels. The parameter $d$ in Equation (2.10) is the channel diameter. The first fraction of the right hand side can be considered as an effective thermal conductivity since in this case the heat transfer occurs via internal convection. Important to note is the geometry dependency of the effective thermal conductivity term:

$$
k_{e f f}=f(d, T)
$$

\section{Mutual Friction}

When a certain heat flux density is exceeded the temperature increase shows a cubic dependence on the heat flux density. To explain this experimental observation Gorter and Mellink proposed a mechanism they called mutual friction [25, 26]. Gorter and Mellink considered mutual friction between the two in opposite direction 
flowing components of He II as the cause of the additional thermal resistance and after some refinements derived the following mutual friction term:

$$
F_{s n}=A_{G M} \rho_{n} \rho_{s}\left(v_{s}-v_{n}-v_{0}\right)^{2}\left(v_{s}-v_{n}\right),
$$

where $A_{G M}$ is a temperature dependent mutual friction coefficient, also known as Gorter-Mellink-Coefficient and $v_{0}$ is a small velocity offset to improve the overall quality of the model. In order to arrive at this conclusion Gorter and Mellink made temporal and spatial averages of the two respective velocities, neglecting the different flow profiles of the two components due to the non equal viscosities. This formulation of a mutual friction term is thus only macroscopically valid. Due to the made assumptions the approach of Gorter and Mellink is strictly speaking only valid in case of steady state conditions and two equations of motion can be written as:

$$
0=-\frac{\rho_{s}}{\rho}\langle\nabla p\rangle+\rho_{s} s\langle\nabla T\rangle-F_{s n},
$$

for the superfluid component and

$$
0=\mu_{n} \nabla^{2}\left\langle v_{n}\right\rangle-\frac{\rho_{n}}{\rho}\langle\nabla p\rangle-\rho_{s} s\langle\nabla T\rangle+F_{s n},
$$

for the normal fluid component, with $\langle\cdots\rangle$ indicating the temporal and spacial averaged values.

\section{Quantum Turbulence in Helium II}

In an experiment conducted by Osborne where he rotated a bucket filled with He II at $1 \mathrm{~K}$ he found that the surface of the liquid had a parabolical shape [27]. This was surprising as the liquid at $1 \mathrm{~K}$ consists mostly of superfluid component, which does not experience any drag between the rotating wall and the bulk liquid. To explain this apparent discrepancy between the measurement results and the theory, L. Onsager [28], F. London [29] and R.P. Feynman [30] suggested the existence of quantised vortex lines in the bulk liquid. Between these vortices the superfluid velocity is zero thus fulfilling the condition of the curl free superfluid velocity but still explaining the measurement result from Osborne by having friction between the vortices and the wall. The existence of these quantised vortex lines was proven later by Williams and Packard [31] in 1974 and by Bewley in 2006 [32] with two different methods using different tracer particles. Each of the quantised vortices contains a unit of circulation $\kappa=\frac{h}{m}$ where $h$ is the Plank constant and $m$ the mass of a helium atom such that $\kappa=9.97 \times 10^{-8} \mathrm{~m}^{2} \mathrm{~s}^{-1}$.

For steady state conditions Hall and Vinen assumed a homogenous distribution of the quantised vortices and an average vortex velocity of zero and arrived at the following expression for the dissipative mutual friction term as a function of the vortex line density [33, 34]:

$$
F_{s n}=\frac{B_{H V} \rho_{s} \rho_{n} \kappa}{2 \rho} \frac{2}{3} L_{0} v_{s n},
$$


with $v_{s n}=v_{s}-v_{n}$ the relative flow velocity. Here $B_{H V}$ is the temperature dependent Hall-Vinen-Coefficient and $L_{0}$ the average vortex line length per unit volume. With this formulation Hall and Vinen connected the macroscopic mutual friction approach of Gorter with the idea of microscopic quantised vortices of Feynman.

For the vortex line density Vinen derived the following expression:

$$
\frac{d L}{d t}=\frac{d L}{d t}_{p}-\frac{d L}{d t} d
$$

where the first term on the right-hand side is the production term of quantised vortices and the second term on the right-hand side is the destruction term of quantised vortices. Based on dimensional arguments Vinen found the following expression for the production term [33, 34]:

$$
\frac{d L}{d t}_{p}=\frac{1}{2}_{2} \xi_{1} B_{H V} \frac{\rho_{n}}{\rho} L^{\frac{3}{2}} v_{s n},
$$

and for the destruction term:

$$
\frac{d L}{d t}_{d}=\xi_{2} \frac{\kappa}{2 \pi} L^{2}
$$

The two parameters $\xi_{1,2}$ are of temperature dependent phenomenological nature. With this description a calculation of the steady state vortex line density for steady state experiments is possible with some limitations [33, 34].

For large channels the influence of the wall on the vortex production and annihilation is negligible and Vinens original approach is valid. For small channel diameter the influence of the wall can not be neglected as was shown by M. Sciacca et al. 35. They considered the influence of the wall and derived the following modified equations where they also include a geometry dependent parameter characterising the influence of the wall on the vortex dynamics:

$$
\frac{d L}{d t}=-\beta_{v} \kappa L^{2}+\left[\alpha_{0} v_{n s}-\omega^{\prime} \beta_{v} \frac{\kappa}{d}\right] L^{\frac{3}{2}}
$$

where $\alpha_{0}, \omega^{\prime}$ and $\beta_{v}$ are dimensionless temperature and geometry dependent coefficients related to the production and the destruction of vortices. The steady state non-zero solution of this equation is

$$
L^{\frac{1}{2}}=\frac{\alpha_{0}}{\beta_{v} \kappa} v_{s n}-\frac{\omega^{\prime}}{d} .
$$

This equation has positive solutions for values of $v_{s n}$ higher than $v_{c 1}=\frac{\beta_{v} \kappa \omega^{\prime}}{\alpha_{0} d}$.

Different experiments conducted with a broad range of channel dimensions and over a broad temperature range have shown that in helium II three distinct turbulent states exist [36, 37]. Two of the turbulent states called TI and TII occur in round and small aspect ratio channels whereas a third turbulent regime named TIII occurs in wide aspect ratio channels. The physical nature of the three turbulence states in He II is an ongoing topic of research. 
For the turbulent states TI and TII Sciacca et al. 35] derive the following expressions

$$
\begin{aligned}
L^{\frac{1}{2}} & =\frac{\gamma_{T I}}{\kappa} v_{n s}-1.48 \frac{\alpha_{1}}{d} \\
L^{\frac{1}{2}} & =\frac{\gamma_{T I I}}{\kappa} v_{n s}-1.48 \frac{\alpha_{2}}{d}
\end{aligned}
$$

for the vortex line density, which is a solution of Vinen 38. modified by the last term. The parameters $\gamma_{T I}, \gamma_{T I I}, \alpha_{1}$ and $\alpha_{2}$ have been determined numerically for a limited range and so far no analytic expression as a function of diameter and temperature has been given.

Experimental investigations of the critical velocities for the transition between the laminar and the turbulent state TI and between the turbulent states TI and TII have shown that the transition between the laminar state and the turbulent state TI occurs for an average vortex line separation of $L^{\frac{1}{2}} d \simeq 2.5[39$. This transition also appears to have a considerable hysteresis and laminar behaviour has been found at vortex line density higher than the above given critical value. For the second transition between turbulent state TI and TII an average vortex line separation of $L^{\frac{1}{2}} d \simeq 12$ has been identified [39]. This transition does not show any hysteresis and the transition occurs immediately when the critical value is exceeded. The diameter dependent nature of both relations indicate the interaction of the vortex lines with the wall as the dominant factor for the development of turbulence.

\section{Quantum Reynolds Number}

Jou et al. introduced the concept of a quantum Reynolds number to describe He II heat and mass transfer [40. In classical fluid dynamics a flow condition is characterised by the Reynolds number $R e=\frac{v d}{\nu}$ taking into account the geometry and kinematic viscosity to characterise the ratio of the inertial forces to the viscous forces. In the case of the two fluid model a Reynolds number in the classical sense can only be determined for the normal fluid component, which is associated with a non-zero viscosity. For the superfluid component no classical Reynolds number can be determined. To resolve this the authors suggest the use of the quantum of vorticity $\kappa$. In this way the Reynolds number characterises the relation between the mutual friction and the wall interaction similar to the commonly used definition of the Reynolds number. Since the onset of turbulence in He II is related to the relative flow velocity $v_{n s}=v_{n}-v_{s}$ the authors define a quantum Reynolds number in [40] as

$$
R e_{q}=\frac{v_{n s} d}{\kappa}
$$

For zero-net mass flow conditions the quantum Reynolds number can be directly linked to the transported heat flux density using Equation 2.5): 


$$
R e_{q}=\frac{d}{\kappa \rho_{s} s T} q
$$

The concept of a quantum Reynolds number makes it possible to define a critical Reynolds number for the transition between different heat transfer regimes. The critical Reynolds number accounts for a geometrical, thermal and heat flux dependency unifying different other approaches to the prediction of the onset of turbulence.

\section{Quantum Reynolds number and Quantum Turbulence}

Combining the vortex line approach to turbulence in He II with the concept of a quantum Reynolds number gives the following expression for the quantum Reynolds number:

$$
R e_{q}=\frac{L^{\frac{1}{2}} d+1.48 \alpha_{1}}{\gamma_{T I}}
$$

in the case of TI turbulence.

Using the concept of a critical Reynolds number for the transition from the laminar to the TI regime and the transition from TI to TII Sciacca et al [35] derive the following model for the heat transfer in He II:

$$
\Delta T=\frac{8 \mu_{n} l}{\pi r^{4} \rho^{2} s^{2} T} \dot{Q} \text { for } \quad R e<R e_{1},
$$

for the laminar regime, where $l$ is the length of the channel, $r$ is its radius and $R e_{1}$ the critical Reynolds number for the development of the TI turbulent regime. The critical Reynolds number $R e_{1}$ can be determined from Equation 2.25) and the average vortex line density $L^{\frac{1}{2}} d$ for transition between the laminar and the TI turbulent regime as mentioned before in Section 2.3.1.

This leads to the following equation for the turbulent regimes TI and TII:

$$
\Delta T=\frac{8 \mu_{n} l}{\pi r^{4} \rho^{2} s^{2} T} \dot{Q}+\frac{K l}{\zeta}\left[\frac{\gamma_{0}}{\kappa \rho_{s} s T} \frac{\dot{Q}}{\pi r^{2}}-\frac{\omega^{\prime}}{2 r}\right]^{2} \frac{\dot{Q}}{\pi r^{2}} \quad \text { for } \quad R e>R e_{1},
$$

where $\zeta=\frac{\rho T s^{2} \rho_{s}}{\rho_{n}}$ and $K=\frac{1}{3} \kappa B_{H V}$. The model parameters $\gamma_{0}$ and $\omega^{\prime}$ are temperature and geometry dependent and change depending on the turbulence regime TI or TII change as follows:

$$
\begin{gathered}
\gamma_{0}=\frac{\gamma_{T I}+\gamma_{T I I}}{2}\left(1+\frac{\gamma_{T I I}-\gamma_{T I}}{\gamma_{T I I}+\gamma_{T I}} \tanh \left[A\left(R e-R e_{2}\right)\right]\right), \\
\omega^{\prime}=\frac{1.48 \alpha_{1}+1.48 \alpha_{2}}{2}\left(1+\frac{\alpha_{2}-\alpha_{1}}{\alpha_{1}+\alpha_{2}} \tanh \left[A\left(R e-R e_{2}\right)\right]\right),
\end{gathered}
$$

where $A=\frac{1.47 \kappa}{d\left(v_{\text {edge }}-v_{c} 2\right)}$ and $R e_{2}$ is the critical Reynolds number for the development 
of the TII turbulent regime. The critical Reynolds number $R e_{2}$ can be calculated from Equation 2.25 and the critical vortex line density for the transition between the turbulent regime TI and TII mentioned in Section 2.3.1.

The parameter $A$ defines the width of the transition between the turbulent states TI and TII by taking into account the counterflow velocity between the onset of the transition, traditionally denoted by the index $c 2$, and the fully developed turbulent state TII.

The TIII regime found in large aspect ratio channels is not very well investigated and the question remains if the TIII is actually a state similar or equal to the TI or TII state [39. Due to the lack of experimental data, the turbulent state TIII will not be considered any further in this work.

As can be seen from the model the authors use the same idea to model the two turbulent states with different parameters. They also give values for their parameters for a specific experiment and achieve a remarkably good fit to the experimentally measured vortex line density. Even with this approach to the vortex line density the real nature of the turbulence in helium II is still an open question of research and discussion.

\subsubsection{Heat Transfer in Helium II}

The previously described theoretical approaches can be used to model the heat transfer in the laminar regime using the following equation:

$$
\dot{q}=-\frac{d^{2}(\rho s)^{2} T}{\beta \mu_{n}} \frac{d T}{d x} .
$$

For the fully turbulent regime Gorter and Mellink gave the following expression when neglecting the fountain effect [25].

$$
\nabla T=-\frac{A_{G M} \rho_{n}}{\rho_{s}^{3} s^{4} T^{3}} \dot{q}^{3} .
$$

For the modelling the heat flux density is needed as a function of the temperature gradient. When doing this step it is necessary to define the interpretation of the vector $\nabla T$ to the power of $\frac{1}{3}$, which appears in the equation solved for the heat flux density:

$$
\dot{q}=-\left(\frac{\rho_{s} s^{4} T^{3}}{A_{G M} \rho_{n}}\right)^{\frac{1}{3}}(\nabla T)^{\frac{1}{3}} .
$$

To resolve this the following interpretation is used:

$$
(\nabla T)^{\frac{1}{3}}=(|\nabla T|)^{-\frac{2}{3}} \nabla T .
$$

where the first factor of the right hand side results in scaling of the temperature gradient and the second factor maintains the direction of the vector. Since this treatment maintains the original direction of the temperature gradient it can also be applied not only in the one dimensional case but is also valid in higher dimensional cases. 
If Equation 2.33 is taken into account in Equation 2.32 the following expression is obtained:

$$
\dot{q}=-\left(\frac{\frac{\rho_{s} s^{4} T^{3}}{A_{G M} \rho_{n}}}{(\nabla T)^{2}}\right)^{\frac{1}{3}} \nabla T .
$$

In this case the first factor of the right hand side can then be regarded as an effective thermal conductivity. This effective thermal conductivity is dependent on the local gradient, another extraordinary property of helium II. An investigation by Sato et al. [41] showed that an exponent of 3.4 fits thermal conductivity data over a very wide range better than the exponent 3 , which was originally derived by Gorter and Mellink [25]. Thus from here on the exponent 3.4 is used. The fluid properties of He II that characterise the effective thermal conductivity are summarised by a factor $f^{-1}$, which was determined by a fitting process by Sato et al.:

$$
\dot{q}=\left(\frac{f^{-1}}{\left(\frac{\Delta T}{l}\right)^{2.4}}\right)^{\frac{1}{3.4}} \frac{\Delta T}{l} .
$$

The $f^{-1}$-function has a peak at a temperature of $1.9 \mathrm{~K}$. At this temperature the effective thermal conductivity in the turbulent regime is the highest, for an increasing temperature the function decreases drastically until it reaches zero at the lambda line. For temperature lower than the peak temperature the value of the $f^{-1}$-function is decreasing. Figure 2.6 plots the $f^{-1}$-function for saturation conditions in the temperature range from $1.5 \mathrm{~K}$ to $2.175 \mathrm{~K}$ as calculated by Sato et al. 41.

\subsection{Application of Helium Heat Transfer to Accelerator Magnet Cooling}

The cooling of the superconducting magnets was a focus point for the LHC study group charged with the design for the LHC. First experimental investigations of the heat transfer by $\mathrm{C}$. Meuris revealed that the electric insulation is the main thermal barrier for the cable cooling [42. This first investigation also found that the polyimide wrapping is not a solid barrier but that it is permeable to He II, which substantially improves the heat transfer. Initially the insulation of the cable was supposed to be made from a two layer wrapping of polyimide and a third insulation layer made from a glass fibre cloth impregnated with epoxy. The study experimentally investigated the influence of the helium bath temperature and also identified the main heat transfer paths that contribute to the cooling of the sample. The obtained experimental data is explained qualitatively but not quantitatively due to the overlapping contributions of the different channels. Overall it was found that an insulation of this type provides sufficient cooling for the expected heat loads during the operation of the LHC. 


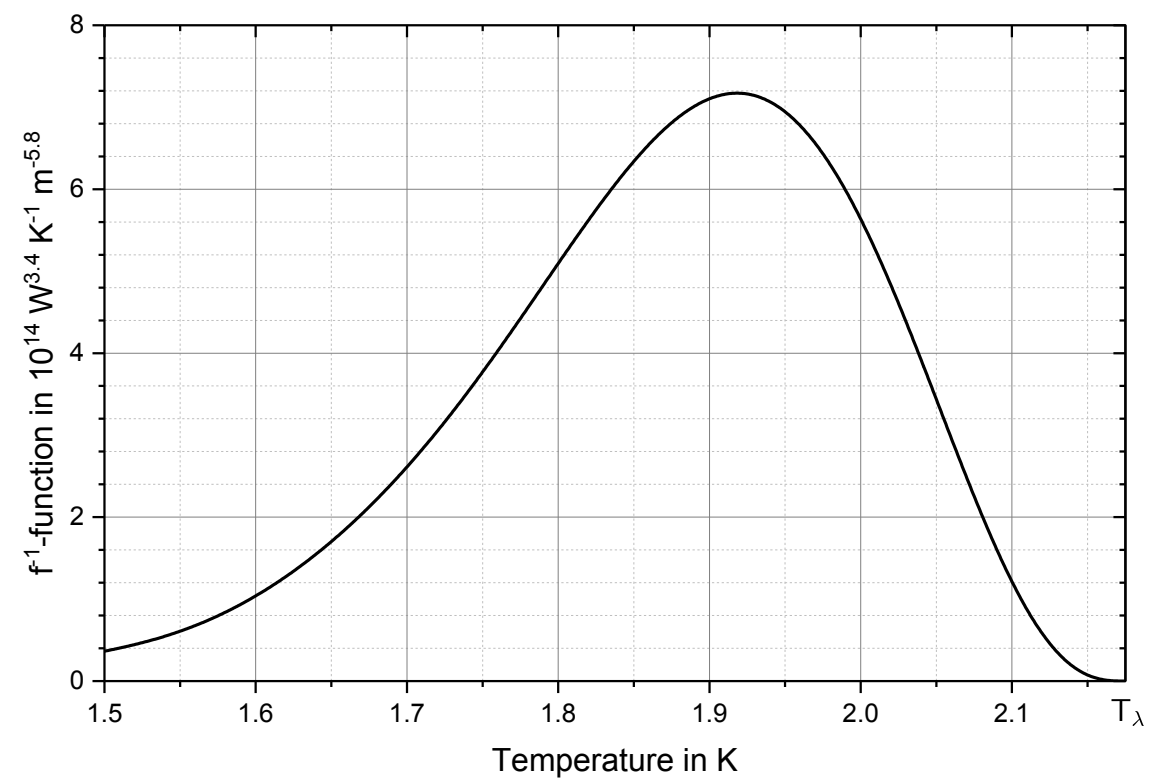

Figure 2.6. The $f^{-1}$-function as calculated by the fit from Sato et al. for He II saturation conditions [4]. The characteristic peak at $1.929 \mathrm{~K}$ is the temperature with the highest thermal conductivity in He II in the turbulent heat transfer regime for a given temperature gradient.

Based on the works of C. Meuris the group at CEA Saclay continued the investigation. Baudouy et al. 443, 44] developed a one dimensional experiment to investigate the steady-state heat transfer through different types of insulation patterns at $1.9 \mathrm{~K}$. The primarily changed parameter was the third layer composition. Three different types of third layer wrapping material were investigated, an adhesive polyimide wrapping, a fibre glass alternatively woven with glass-Kevlar and thirdly a polyimide-Kevlar woven layer. A theoretical model was also developed for the heat transfer in the different samples, where a porosity factor is introduced to scale the effective thermal conductivity of He II in the turbulent regime. Due to the one dimensionality of the experiment only one global helium channel is used for the calculation. Overall a deviation of less than $10 \%$ between the measurement data and the theoretical modelling proves the general idea for the steady-state behaviour of the model.

B. Baudouy and C. Meuris [45, 46, 47] also report on the results of an experimental investigation on the steady-state heat transfer between a heated cable stack and the He II coolant bath. They study a broad range of different insulation patterns for a wide bath temperature range and characterise the influence of the different insulation patterns on the heat transfer. Baudouy concludes the experimental investigation by suggesting an insulation pattern based on tapes consisting of 
polyimide only. In the modelling part of his $\mathrm{PhD}$ thesis B. Baudouy 45] models the solid conduction contribution of the polyimide wrapping and the helium channel contribution. He numerically investigates different sample geometries by varying them in a parametric sweep and the results indicate that an internal He II volume contributes significantly to the temperature homogenisation inside the cable. Furthermore they confirm the hypothesis of C. Meuris that the channels formed by the insulation wrapping that connect the helium voids to the bath contribute significantly to the heat transfer.

In a separate modelling effort B. Baudouy reports that the helium channels in the insulation and the bulk insulation can be modelled as parallel heat transfer paths in the temperature range $1.7 \mathrm{~K}$ to $1.9 \mathrm{~K}$ [45]. For higher bath temperatures the two heat transfer paths actually become coupled and heat is transferred between the two paths such that part of the heat flux initially transported by the helium channel is transported by the solid insulation. The change in heat flux amounts up to $10 \%$ at $2.1 \mathrm{~K}$. Overall he states that the simple assumption of parallel uncoupled heat transfer paths is a valid first order model for the thermal characteristics.

In their study on the heat transfer in Rutherford-type superconducting cables Kimura et al. 48, found that the heat transfer is dominated by channels in the insulation that are in the turbulent heat transfer regime. For higher heating powers they state that the turbulent regime is not fully sufficient to explain the thermal characteristics of the insulation. In their investigation they found a maximum heat transfer capacity of $18 \mathrm{~mW} \mathrm{~cm}^{-3}$ for a bath temperature of $1.9 \mathrm{~K}$ before the temperature rises above the lambda temperature. Their investigation focused on an insulation pattern composed of two layers where the inner layer consists of two identical polyimide tapes with a thickness of $25 \mu \mathrm{m}$ and the outer layer of a single $50 \mu \mathrm{m}$ thick polyimide tape with a space between turns of $2 \mathrm{~mm}$ an insulation that differs from the LHC's final dielectric insulation in which the insulation tapes have a thickness of $50 \mu \mathrm{m}$ for the inner two layers and $69 \mu \mathrm{m}$ for the outer layer.

M. La China et al. [49, 50, focus on the theoretical improvement of the LHC insulation pattern such that the heat transfer capability is greatly improved and the magnets can sustain higher heat loads in steady-state operation. When comparing the LHC dipole insulation, which was chosen based on the experimental investigations of B. Baudouy, with the newly designed insulation pattern, the new insulation pattern shows an improvement of a factor of 10 over the state of the art insulation as used in the LHC. This improvement is obtained by creating more and wider helium channels in the insulation compared to the standard LHC insulation pattern.

An insulation based on the works of La China [49, 50] was investigated experimentally by P.P. Granieri [51, 52, 53. This enhanced insulation is a proposal for the inner triplet magnets, that are closest to the interaction region, that are being designed for the high luminosity upgrade of the LHC. Due to the higher heat loads that go along with an increased luminosity a new insulation has to be found that greatly improves the thermal contact between the cable and the helium bath. The experimental set-up is based on the idea of C. Meuris 42] and uses a stack of mock-up cables to model a part of the coil winding. Granieri found that the new insulation pattern at a compression of $100 \mathrm{MPa}$ improves the thermal contact 
by a factor four compared to the classic dipole insulation. At the same time the new insulation pattern shows a higher dependency on the transversal compression of the cable stack where a lower pressure increase the thermal contact greatly. In the case of one heated cable he found that the thermal decoupling between the heated cable and the adjacent cables is considerably improved for the enhanced insulation and the adjacent cable shows only a minor temperature change when the neighbouring cable is heated. This is in contrast to the classical insulation where the decoupling is not very strong and also the adjacent cables show a considerable temperature increase when the neighbouring cable is heated.

In his modelling approach to the cooling of the cable P.P. Granieri distinguishes between the channels in the small face of the sample, which directly face the helium bath and the channels on the broad face of the cable, which are compressed by an experimentally varied pressure. This model also accounts for solid conduction through the insulation. He found that the channel dimensions in the third insulation layer on the broad face of the cable is changing considerably depending on the compression contrary to the channel diameter he deduces for the channels on the small face of the cable.

In another study P.P. Granieri et al. [54] compare the heat transfer for three different cables used in the LHC with their respective insulation wrapping. He found that the insulation used in the main focusing magnets of the LHC has the worst heat extraction capability with $24 \mathrm{~mW} \mathrm{~cm}^{-3}$ for a temperature difference of $50 \mathrm{mK}$, second to the thermal extraction capability of the MQXA inner triplet magnet with $27 \mathrm{~mW} \mathrm{~cm}^{-3}$ and finally $32 \mathrm{~mW} \mathrm{~cm}^{-3}$ for the main bending magnets of the LHC.

\subsection{Thesis Objectives and Outline}

Section 2.4 summarises the investigation of the heat transfer between a superconducting Rutherford cable and the helium bath from the beginning of the LHC design until the recent development of the insulation patterns for the high luminosity upgrade of the LHC. All of the studies relied on the stack method developed by C. Meuris to simulate a part of the magnet winding [42]. Common to all above mentioned studies is the use of a replacement cable fabricated from a normal conducting material such as steel or copper nickel wires and cables. This choice together with the insulation patterns, which were all made by hand, give a first approach to the thermal contact between the cable and the helium bath. To gain a complete picture of the thermal characteristics of the thermal contact the use of a large scale production cable and machine made insulation is the next necessary step. A step that is investigated in the present work.

The conducted study focuses on the experimental investigation of the heat transfer of a superconducting Rutherford cable and includes experimental data not only for the steady-state but also the transient condition. The transient cooling is becoming increasingly important at higher collision energies since with the increased energy the temperature margin of the superconductor is decreasing while at the same time the transient heat loads from beam-beam interactions and other 
sources are increasing. This work is addressing this issue by analysing the temporal evolution of the recorded measurement data.

The next chapters deal with the different components used in this study. In Chapter 3 the experimental set-up and its components are discussed together with their measurement resolution and their uncertainty on the experimental data. Chapter 4 deals with the developed heating system and details on the determination of the amount of deposited heat together with the uncertainties that arise from the calorimetric measurements. A model for the steady-state heat transfer between the superconducting Rutherford cable sample and the He bath is developed in Chapter 5. It uses a newly developed direct transition model for the effective thermal conductivity of He II to calculate the effective thermal conductivity. The results of the experimental measurements and the model are shown in Chapter 6 firstly for steady-state conditions and secondly for transient conditions where the behaviour of the time constant is discussed in detail. In Chapter 7 the results of the study are summarised and conclusions are drawn on the impact of the transient heat transfer on the cooling of superconducting Rutherford cables. 



\section{Experimental Set-up}

A crucial point of this thesis is the experimental investigation of the cooling of a superconducting cable as it is used in the LHC. The study of the cooling of the superconducting cable has been going on for more than 20 years. Starting with the research into the technologies necessary for the construction of the LHC, efforts were made to find solutions to the challenges that arise from the task of building a cutting edge accelerator.

Most of the research into the cooling of superconducting cables has been made with mock-up cables fabricated out of a non-superconducting material that has the same geometric dimensions as the original superconducting cable. Usually only small lengths of the cable have been fabricated for the experiments. This way of investigating the thermal behaviour is a valid first order approach since the main thermal barrier that limits the steady-state cooling of cables is the dielectric insulation.

Using these mock-up cables and not the superconducting cable itself means that due to the short length of the manufactured cable the insulation has to be wrapped around the cable manually. Applying three layers of cable insulation manually implies a certain non-uniformity and inconsistency in terms of the pattern and the mechanical stress in the insulation layers. It is thus desirable to investigate the cooling on a machine wrapped superconducting cable sample that is taken right from the production street that produces the cable as it is used in the final application.

This chapter describes the development of a laboratory scale experiment with the aim of measuring the steady-state and transient cooling of a superconducting cable. First a detailed treatment of the set-up with the different components is given. This is followed by a description of the heating system and the source of the generated heat in the superconducting cable. And lastly the data analysis used for the interpretation of the measurement data is explained.

\subsection{Measurement Procedure}

With the aim of measuring a superconducting cable a new idea of generating heat in the sample had to be found. Joule heating where current is injected into the cable is not generating any heat since the superconductor has no measurable resistance and as a consequence no heat is generated in the cable when transporting a current. This holds true for direct current conditions where the superconductor is ideal and no losses occur. This changes when the current is not a direct current but an alternating current. For AC conditions the superconductor does not behave ideal anymore and losses occur. These currents can be created by different means, either 
an $\mathrm{AC}$ current is directly injected into the cable or the cable can be exposed to an alternating magnetic field, which then induces AC currents generating losses in the cable.

With the idea of using AC loss to generate heat inside the cable a set-up was developed. In order to characterise the heat transfer the He bath temperature as well as the temperature in the sample is measured as a function of time. Using the separately determined generated heat together with the temperature recording the heat transfer between the sample and the He bath is characterised.

\subsection{Experimental Set-up}

The goal of the experimental set-up was to enable heat transfer measurements without having to resort to a full size, $15 \mathrm{~m}$ long and $27.5 \mathrm{t}$ heavy, magnet. This work focuses on the heat transfer between the superconducting cable and the static pressurised He bath and does not take the whole heat transfer path up to the heat exchanger pipe into account (compare to Figure 2.2). This approach was chosen since the thermal contact inside the pressurised He bath is characterised by a continuous large area connection and the main bottleneck for the heat transfer is posed by the polyimide wrapping around the cable. Focusing on this element the experiment can be reduced drastically in size. To represent the coil winding in a magnet and to reproduce the boundary conditions it was decided to take several superconducting Rutherford cables and stack them upon each other. This sample geometry is a representation of the coil winding pack where only the small face of the conductor is in direct contact with the He bath, something that is also achieved by stacking the cables. Figure 3.1 shows an illustration of the sample, consisting of four cable stacked upon each other on the broad side. Details in this figure are explained in the following paragraphs.

Figure 3.2 shows an illustration of the set-up and identifies the key components. The heat in the sample is generated with a superconducting coil whose current is varied in a harmonic manner to generate an oscillating magnetic field. In order to measure the sample in pressurised He II a vessel is placed inside the magnet bore. This vessel is cooled with the help of a copper heat exchanger at the top end of the vessel. Inside the vessel is the sample holder, which keeps the sample in the centre of the magnet such that the maximum heat generation in the sample is guaranteed. The instrumentation of the sample is also shown schematically. Each of the main components of the set-up is dealt with individually in the next subsection starting with the sample, increasing in geometric scale to the sample holder, the vessel and finally the heating system. Especially the heating system is explained in detail and the amount of the generated losses is described in detail.

\subsubsection{Sample}

For a laboratory scale experiment the superconducting coil package is represented by a stack of four superconducting Rutherford cables. 


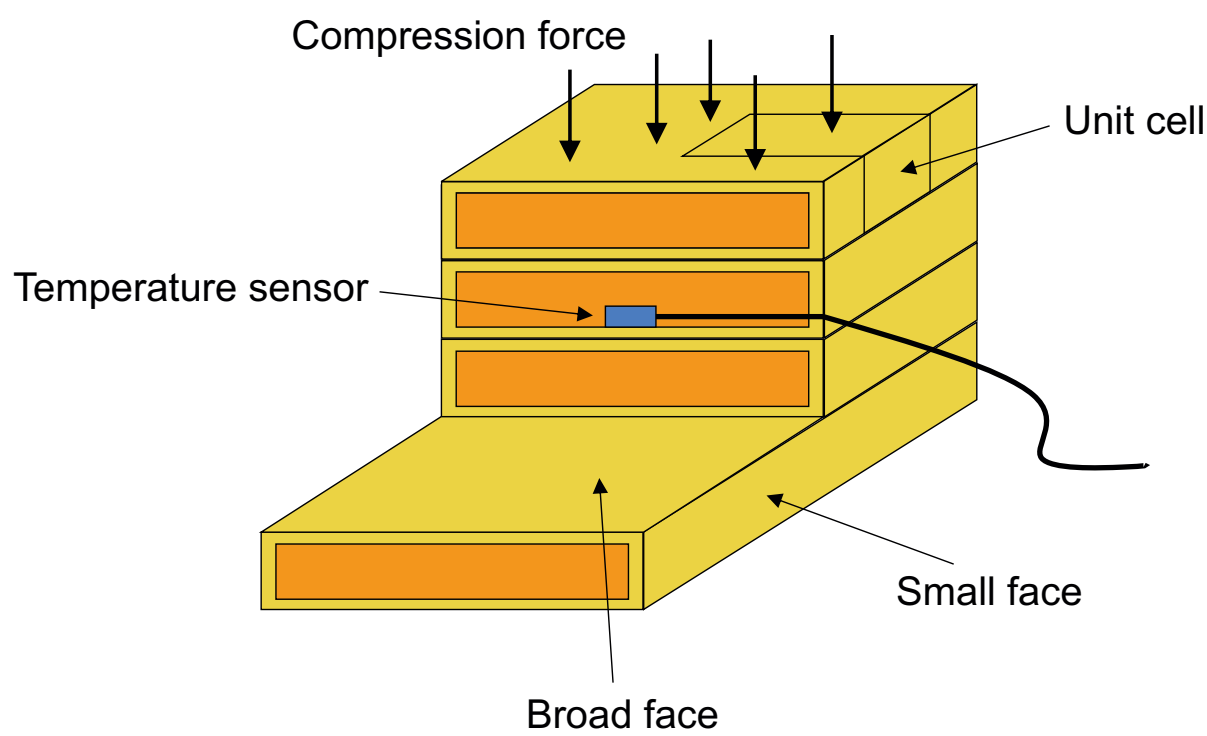

Figure 3.1. Illustration of the sample after assembly. The sample consists of four cables where one of the two centre cables is instrumented with two temperature sensors. The cables are stacked and compressed on the broad face and the small face of the cable is facing the He bath. A unit cell is also indicated, which represents a repetitive unit in a cable. The unit cell will become important for the modelling of the heat transfer between the cable and the He bath.

\section{Superconducting cable}

In the present work a sample from the cable of the inner layer of the LHC main bending magnets is investigated because it is subject to the highest heat loads as shown in Section 2.2. For the main bending magnets two different cables are used, both having the same transport current but the cable dimensions are depending on the environment the cable is exposed to. The inner layer cable is exposed to a higher heat load and it was decided to focus the investigation on this cable. The dimensions of the inner layer cable are given in Table 3.1 with the interested reader being referred to the LHC design report for the dimensions of the outer layer cable [4]. The inner layer cable consists of 28 strands each with a nominal diameter of $1.065 \mathrm{~mm}$ that are twisted with each other and then rolled into a trapezoidal shape. The shape of the conductor is based on the requirements of the application and since AC loss are undesired the cable has been optimised for low AC loss [55].

\section{Sample instrumentation}

To measure the temperature of the sample temperature sensors need to be placed inside the sample. As can be seen from Table 3.1 the dimensions of the strand are 


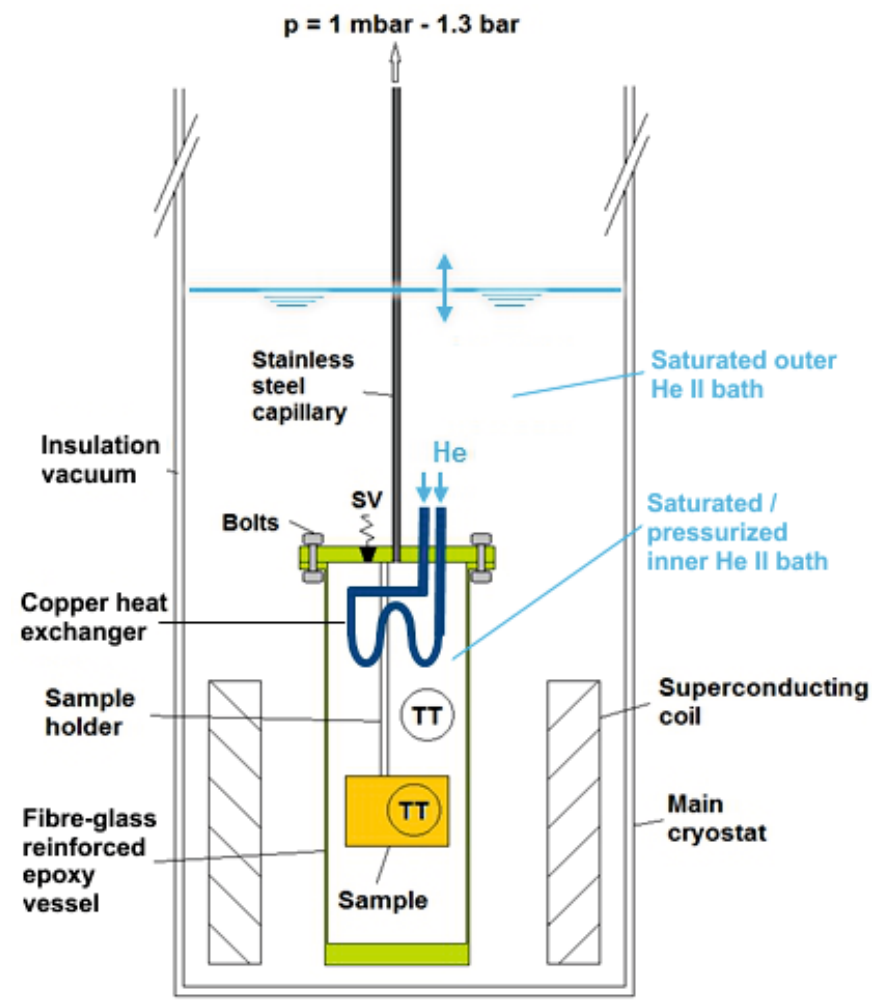

Figure 3.2. Schematic of the measurement set-up showing the different components and their position in the cryostat. The safety valve is labelled with SV, the temperature sensors are labelled TT. The copper heat exchanger is an open loop, with both ends open to the outer He bath. It increases the available surface area and thus helps to maintain a stable inner He bath temperature. The inner He bath can be changed between saturated and pressurised conditions by opening or closing the safety valve manually.

in the order of $1 \mathrm{~mm}$ and a minimum size sensor has to be used so that the internal structure of the cable is changed in the smallest extent possible. With most sensors having dimensions in excess of $2 \mathrm{~mm}$ the choice for the temperature sensors is very limited. Based on these requirements a bare-chip Cernox ${ }^{T M}$ temperature sensor was chosen as temperature instrumentation of the sample [56]. This sensor has dimensions of $0.965 \mathrm{~mm} \times 0.762 \mathrm{~mm} \times 0.203 \mathrm{~mm}$, a footprint small enough such that only one strand in the Rutherford cable has to be altered in order to install the sensor and the connection leads. Since the sensor has no electric insulation at all it needs to be insulated from the cable itself in order to guarantee proper functioning of the sensor. This is achieved by a thin layer of a fast drying two-component epoxy. 
Table 3.1. Strand and cable dimensions as listed in the LHC design report [4].

\begin{tabular}{l|c} 
Rutherford Cable & \\
\hline Number of strands & 28 \\
Mid-thickness at $50 \mathrm{MPa}$ & $1.900 \mathrm{~mm}$ \\
Thin edge & $1.736 \mathrm{~mm}$ \\
Thick edge & $2.064 \mathrm{~mm}$ \\
Width & $15.10 \mathrm{~mm}$ \\
Cable twist pitch & $115 \mathrm{~mm}$ \\
\hline \hline Strand & \\
\hline Diameter & $1.065 \mathrm{~mm}$ \\
Filament diameter & $7 \mu \mathrm{m}$ \\
Number of filaments & $\approx 8900$ \\
Filament twist pitch & $18 \mathrm{~mm}$
\end{tabular}

The connection leads of the sensor were chosen in such a way that a whole strand diameter could be replaced and no extra spaces are created in the cable where the leads are connecting the sensor to the measurement equipment. Figure 3.3 shows the sample after the machining where one half strand is removed from the cable so that it can be replaced with the sensor and its leads. During the machining a small hole is cut in the insulation on the edge of the cable where the leads of the sensor would leave the sample, here the strand is cut on one side. In the centre of the cable a second small hole is made in the insulation on the broad side of the cable in order to cut the strand so that it can be removed by pulling it out on the edge of the cable. The cut pieces of the insulation are kept and later, after the installation of the sensor repositioned to at least partially cover the incision in the insulation.

The sensor is installed by inserting the ends of the leads from the centre of the cable. The pocket where previously the strand had been is filled with a 2-component epoxy to fill the voids around the sensor and make sure that no artificial hole is made where helium can penetrate into the cable and shortcut the insulation. While the glue is still not yet fully cured the leads are pulled through the cavity until the sensor is firmly placed inside the groove. Then the groove is filled with epoxy glue such that no excess glue higher than the cable is left and the saved insulation pieces are replaced on top of the sensor. On the side of the cable where the instrumentation leads are sticking out of the cable additional epoxy glue is placed in order to seal the incision in the insulation and again prevent helium from leaking into the cable without passing through the insulation.

\subsubsection{Sample Assembly}

After the instrumentation of the Rutherford cable with the temperature sensors three additional pieces of the same cable but without instrumentation are stacked upon each other with the instrumented cable being one of the inner two cables. The final sample consists of four cables and has a rectangular shape, see Figure 3.1 


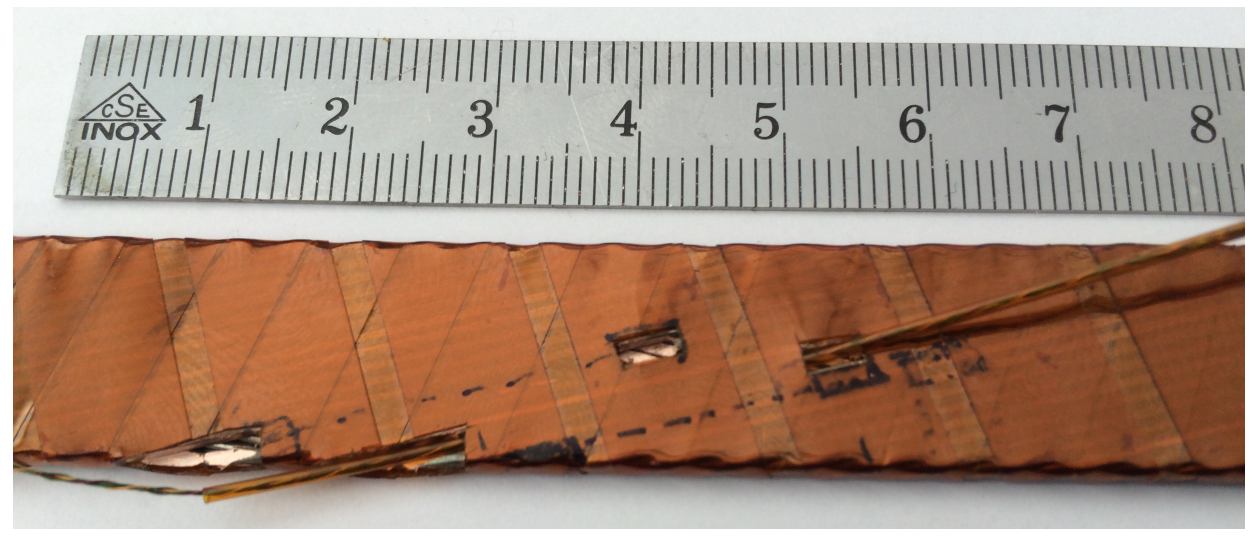

Figure 3.3. Picture of the machined cable with the two pockets where the two sensors are located. One sensor is about to be installed, the wires are already fit through the channel left by the removed strand.

This is done to reduce boundary effect due to cooling via the broad face of the cable and limits the cooling to the small face of the cable where the cable is in contact with the constant temperature He bath. In order to study the effect of compression on the cooling of the sample an insulating fibre glass reinforced epoxy plate is placed on the broad side of the cable stack. This plate has small indents where the heads of the screws that are used to compress the cable stack are positioned, see Figure 3.4 .

The cable itself is insulated from the He bath by the polyimide insulation. The ends of the cable where the cable samples have been cut also need to be insulated, since here the helium voids in the cable would otherwise be in direct contact with the He bath. To insulate the two ends small caps made from fibre glass reinforced epoxy are used. They are filled with epoxy glue prior to their installation. In this way the direct connection is disrupted and the measured temperature is solely dependent on the heat transfer through the polyimide insulation.

Figure 3.4 shows the finished sample after all the assembly steps. The two small wires sticking out of the sample are the instrumentation leads for the two Cer$\operatorname{nox}^{T M}$ sensors each consisting of four Manganin wires in a twisted pair configuration. The sample is instrumented with two sensors in order to have a redundancy for the temperature measurement.

The outer layer of the insulation of the cable is covered in an adhesive that needs to be heat treated to be activated. This heat treatment is done so that the individual turns of the magnet are fixed in their position. This heat treatment is not done in this sample since it could risk the integrity of the installed temperature sensors. 


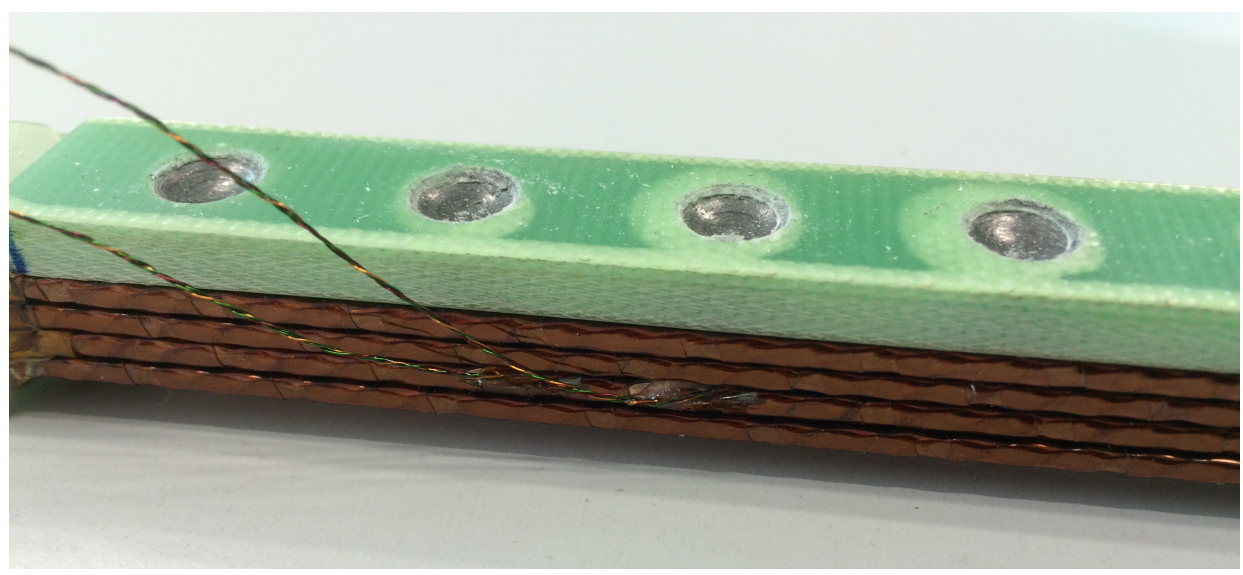

Figure 3.4. Picture of the cable stack consisting of four superconducting Rutherford cables. Clearly visible are two instrumentation wires for the two bare chip temperature sensors leaving the second cable from the bottom. At the top the fibre glass reinforced epoxy plate is visible. The plate is placed in order to avoid cooling of the sample from the top and to homogenise the applied mechanical compression.

\subsubsection{Sample Holder}

In the LHC the cable is mounted under a mechanical pre-compression of $70 \mathrm{MPa}$ before cool-down and during cool-down the thermal contraction decreases the compression of the magnet by an amount between $25 \mathrm{MPa}$ to $40 \mathrm{MPa}$ [4]. With this wide uncertainty of the mechanical pressure at $1.9 \mathrm{~K}$ it is necessary to study the heat transfer at different mechanical pressures.

A limitation of the experimentally achievable compression arises from the heating system that relies on an alternating magnetic field to generate heat. In order to facilitate the penetration of the magnetic field into the sample the sample holder is constructed out of a non-magnetic material. The material chosen for the sample holder is a fibreglass reinforced epoxy. The sample holder has a cylindric shape with a rectangular centre bore where the sample is placed. A set of screws in the sample holder is used to set the compression of the sample by adjusting the torque used to tighten the screws. Six M8 screws are used to press on the fibre glass reinforced epoxy plate mounted on the broad face of the cable and as such influence the mechanical pressure on the sample.

Since the sample holder also experiences thermal contraction between room temperature and cryogenic temperatures it was decided to characterise the sample holder with a dedicated set-up. In this set-up the sample is replaced with a specially prepared copper bar on which six strain gauges are mounted. The rest of the experimental part included polyimide foils and a fibre glass reinforced epoxy plate in order to match the real sample composition and thus its thermal contraction. Knowing the characteristic of the strain gauges at room temperature the mechanical 
pressure on the sample as a function of the applied torque and temperature is measured. By including the changing zero point of the strain gauges as a function of temperature a calibration of the mechanical pressure of the sample is realised.

For the measurement with the real sample it was decided to measure at two different mechanical pressures in the relevant range for the final application. The chosen pressures are $50 \mathrm{MPa}$ and $75 \mathrm{MPa}$. Although higher mechanical pressures are also of relevance but size constraints lead to the sample holder being designed for a maximum pressure of $75 \mathrm{MPa}$ and increasing the pressure over that value would initiate cracks in the material and increase the risk of losing the integrity of the sample holder.

\subsubsection{Pressurised He II Vessel}

Besides the characterisation of the influence of the mechanical pressure on the heat transfer, the experiment also aims to determine the influence of saturated and pressurised He II on the sample cooling. In order to characterise this influence a special vessel was built that creates a secondary He bath in which the He bath pressure can be controlled. Due to the same constraints as for the sample holder this vessel is built from a non-magnetic, non-metallic material to enable maximum penetration of the AC magnetic field.

At the same time this vessel is constructed in such a way that it poses only a small additional thermal barrier. This is necessary since the temperature of the secondary He bath is controlled with the temperature of the primary He bath at saturation pressure. To facilitate this the vessel is designed in such a way that it extends beyond the alternating field into a region where the stray magnetic field is low enough so that a copper heat exchanger could be integrated into the vessel. The heat exchanger shown in Figure 3.5 is essentially an open loop that provides sufficient surface such that the temperature difference between the secondary and the primary He bath is kept well below $2 \mathrm{mK}$ even for the maximum power deposition. This temperature increase is low enough such that the secondary He bath is considered to be at the primary He bath temperature. In this way the two He baths are separated hydrodynamically and still have a good thermal contact.

For instrumentation purposes a feed-through and a capillary are installed in the top fibre-glass reinforced epoxy flange. The capillary is connecting the vessel with the outside of the cryostat. In this capillary all the wires necessary for the temperature sensors in the sample are routed. The capillary also serves as the filling connection at the beginning of a measurement campaign through which helium gas is flowing into the vessel where it is then condensed by means of the copper heat exchanger. A separate feed-through is used for the wiring of a small SMD heater.

For safety reasons and to provide the possibility to experiment in saturated and pressurised He II a valve is integrated into the vessel. The valve is designed to work as a pressure relief valve, which opens at a relative pressure of $0.13 \mathrm{MPa}$ but can also be manually operated from the outside of the cryostat with the help of a stainless steel cord. When experiments are conducted in pressurised He II the valve is closed and the capillary is connected at room temperature to a helium gas 


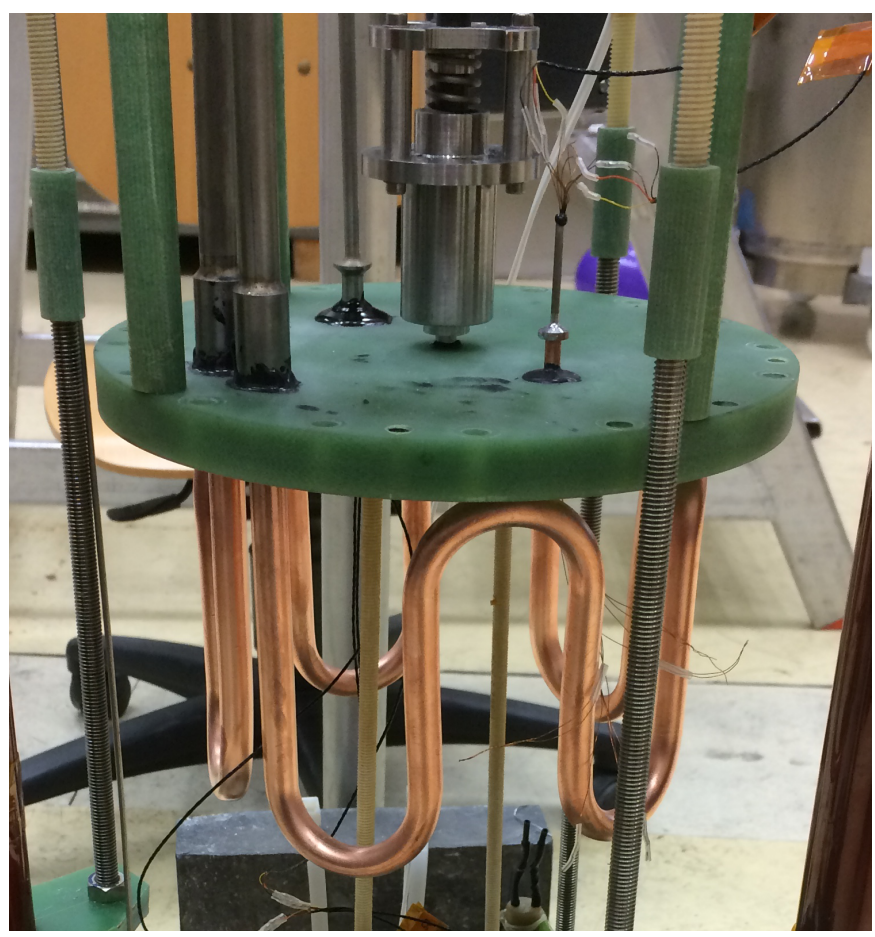

Figure 3.5. Picture of the top flange of the vessel, which contains all the necessary connections. Below the flange the copper heat exchanger can be seen Both ends of the heat exchanger end in the primary He bath above the vessel flange. In the centre of the flange the pressure relieve valve is visible, to the left of the valve is the capillary for the pressurisation and the temperature instrumentation. To the right of the valve is the feed-through for a small heater.

bottle that maintains a constant pressure in the vessel. To operate at saturation conditions the valve is opened and the capillary is connected to the cryostat in order to prevent thermally induced oscillations.

\subsubsection{AC Magnetic Field Sample Heating System}

An alternating magnetic field source is used to generate AC currents in the sample, which then in turn generate heat in the sample. A schematic of the heating system is shown in Figure 3.6. The source of the magnetic field is a superconducting solenoid. The magnet is driven by a $650 \mathrm{~V}$ peak to peak sinus wave source at a constant frequency of $50 \mathrm{~Hz}$. In order to change the amount of deposited power in the sample the current in the magnet is changed by placing additional resistors in series with the magnet. Using the dimensions and inductance of the magnet together with the resistors the magnetic field and its change rate can be calculated. 


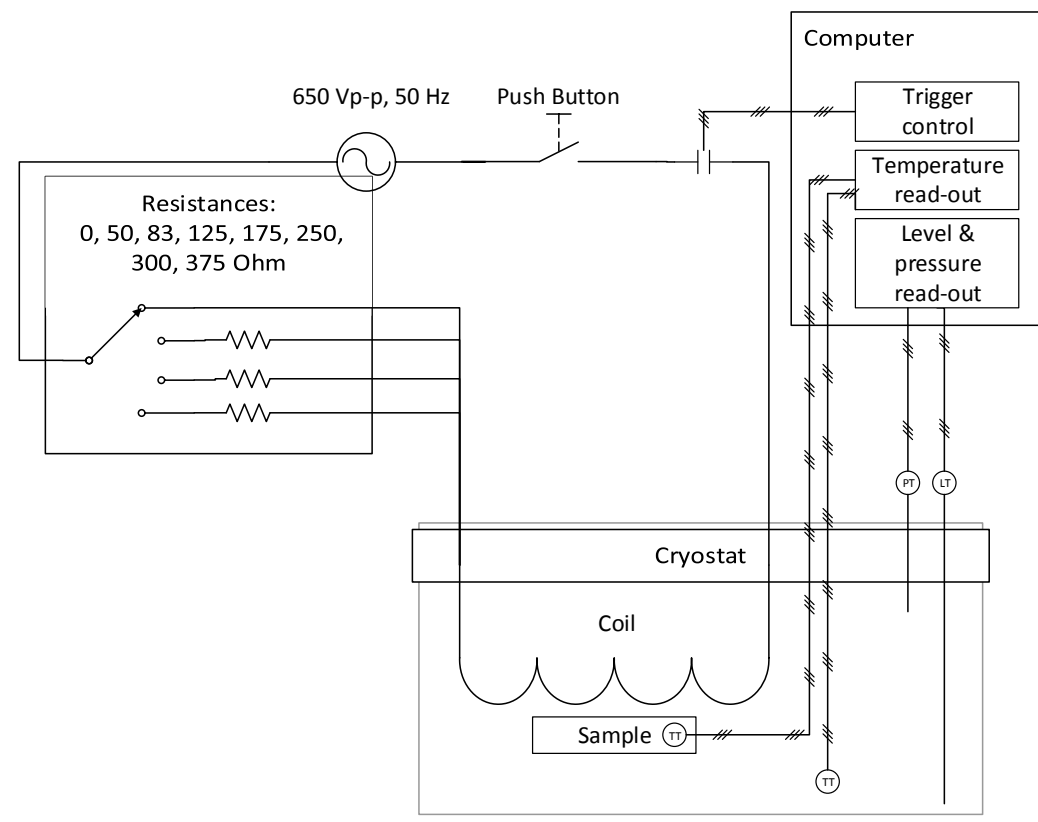

Figure 3.6. Schematic of the heating system of the experimental set-up. It shows the principal components and the different resistances that can be placed in series with the coil in order to change the heating power. It is controlled by an electronic relay that is remotely triggered. The schematics also show the main signals that are recorded during a measurement.

Table 3.2 gives a list of all resistors that can be placed in series with the magnet and the resulting magnetic field.

\section{Alternating current losses}

In order to get an overview of the expected loss levels a short discussion on AC loss and the amount of generated heat is given here. From the known sample dimensions and the values of the magnetic field the AC loss in the cable can be calculated using electrodynamic models.

For the calculation of the AC loss two loss mechanisms have to distinguished [57. The first occurs on the level of the superconducting filaments and is called hysteresis loss. The hysteresis loss is related to flux pinning inside the filament and is dependent on the magnitude of the magnetic field. The second loss type is called coupling loss and occurs on two different geometric scales. The inter-filament coupling loss occurs due to coupling between superconducting filaments inside of one strand. The second occurs on the level of the multi strand cable and is called 
Table 3.2. The utilised resistances and the resulting impedances of the sample heating system. The maximum current in the magnet as well as the calculated magnetic field are listed in additional columns.

\begin{tabular}{l|l|l|l|l} 
Resistance & $\begin{array}{l}\text { Impedance } \\
(0.5 \mathrm{H}, \\
50 \mathrm{~Hz})\end{array}$ & $\begin{array}{l}\text { Maximum } \\
\text { current }\end{array}$ & $\begin{array}{l}\text { Maximum } \\
\text { magnetic } \\
\text { field }\end{array}$ & $\begin{array}{l}\text { Maximum } \\
\text { magnetic field } \\
\text { change rate }\end{array}$ \\
\hline$\Omega$ & $\Omega$ & $\mathrm{A}$ & $\mathrm{mT}$ & $\mathrm{Ts}^{-1}$ \\
\hline \hline 0 & 157 & 2.07 & 10.3 & 3.25 \\
50 & 165 & 1.97 & 9.8 & 3.09 \\
83 & 178 & 1.83 & 9.1 & 2.87 \\
125 & 201 & 1.62 & 8.1 & 2.54 \\
175 & 235 & 1.38 & 6.9 & 2.17 \\
250 & 295 & 1.10 & 5.5 & 1.73 \\
300 & 339 & 0.96 & 4.8 & 1.51 \\
375 & 407 & 0.80 & 4.0 & 1.26
\end{tabular}

cable coupling loss. The coupling losses have a square dependency on the change rate of the magnetic field.

In order to determine the total loss in the cable, the hysteresis loss and each of the coupling losses are calculated individually.

\section{Hysteresis loss}

Hysteresis loss is related to flux pinning inside the superconductor. According to the critical state model a superconductor is a perfect diamagnet and shields its core from the magnetic field by allowing the magnetic field to penetrate into an outer layer depending on the field strength [10. Below a material dependent penetration magnetic field the filament is not fully penetrated and the magnetic field is concentrated in the sheath of the filament. This sheath shields the core of the filament and no field is permitted at the centre. In the sheath currents are induced, which are reversing their direction depending on the orientation of the externally applied field. This alternating current causes the flux centres in the penetrated sheath of the superconductor to change orientation, creating losses in the process. With an increasing field strength the filament becomes increasingly penetrated until it is fully penetrated at its penetration magnetic field. From this it is obvious that the hysteresis loss of a superconductor in an oscillating field depends on the magnitude of the magnetic field with respect to its penetration magnetic field.

According to Y. Iwasa [58] the hysteresis losses can by calculated with the following expression:

$$
\dot{Q}_{h y s}=\frac{2 B^{3}}{3 \mu_{0} B_{p}} f
$$




\section{Experimental Set-up}

with $f$ the frequency of the field oscillation, $B$ the applied magnetic field strength in $\mathrm{T}$ and $B_{p}$ the penetration magnetic field strength in $\mathrm{T}$.

To determine the hysteresis loss first the penetration field strength is calculated. The penetration field strength depends on the conditions of the magnetic field strength but also on the superconductor itself. L. Bottura gives the following expression for the critical current density as a function of magnetic field strength and temperature [8]:

$$
J_{c}=\frac{C_{0}}{B} b^{\alpha}(1-b)^{\beta}\left(1-t^{n}\right)^{\gamma},
$$

where $J_{c}$ is the critical current density, $C_{0}$ a fitting constant, $b$ the reduced magnetic field strength and $t$ the reduced temperature. $\alpha, \beta$ and $\gamma$ describe the dependencies of the reduced magnetic field strength and temperature.

For the reduced temperature Bottura gives:

$$
t=\frac{T}{T_{c 0}}
$$

where $T_{c 0}$ is the critical temperature of the superconductor at $0 \mathrm{~T}$.

The reduced magnetic field strength is given by

$$
b=\frac{B}{B_{c 2}(T)},
$$

where $B_{c 2}$ is given by

$$
B_{c 2}=B_{c 20}\left(1-t^{n}\right),
$$

with $n=1.7$ for the superconductor NbTi. Table 3.3 lists the values for the parameters of the critical current density of NbTi using the known magnetic field strength in the experiment.

Table 3.3. Parameter values for the critical surface of a NbTi superconductor 8 .

\begin{tabular}{c|rl} 
Parameter & Value & Unit \\
\hline \hline$C_{0}$ & 27.04 & $\mathrm{~T} \mathrm{~A} \mathrm{~mm}^{-2}$ \\
$B_{c 20}$ & 14.5 & $\mathrm{~T}$ \\
$T_{c 0}$ & 9.2 & $\mathrm{~K}$ \\
$\alpha$ & 0.57 & - \\
$\beta$ & 0.9 & - \\
$\gamma$ & 2.32 & -
\end{tabular}

The penetration field is calculated with the critical current density at $1.9 \mathrm{~K}$ by:

$$
B_{p}=\mu_{0} \frac{J_{c} d_{f}}{\pi} \approx 0.1 \mathrm{~T},
$$

where $d_{f}$ is the diameter of a filament and $\mu_{0}$ the magnetic permeability. 
The hysteresis loss can then be calculated using the penetration magnetic field strength. The calculated hysteresis loss for all different heating system settings is shown in Table 3.4. The hysteresis loss is in the range from $10 \mu \mathrm{W} \mathrm{cm}^{-3}$ to $280 \mu \mathrm{W} \mathrm{cm}{ }^{-3}$.

Table 3.4. The hysteresis loss calculated with Equation (3.1) for the different heating system powers.

\begin{tabular}{c|c} 
Impedance in $\Omega$ & Hysteresis loss in $\mathrm{mW} \mathrm{cm}^{-3}$ \\
\hline \hline 157 & 0.28 \\
165 & 0.24 \\
178 & 0.18 \\
201 & 0.12 \\
235 & 0.07 \\
295 & 0.03 \\
339 & 0.02 \\
407 & 0.01
\end{tabular}

\section{Coupling losses}

The coupling losses of the superconducting Rutherford cable are distinguished by the geometric scale on which they occur. Increasing in geometric scale the inter-filament coupling loss occurs on the level of a single strand and on the level of a cable the cable coupling loss occurs, which involves at least two separate strands. The coupling losses are of an inductive nature and therefore scale with the square of the applied field change rate. The size of the induction loops determines the time constants of the coupling losses, which causes a Rutherford cable to have two distinct time constants that relate to the different geometric scales.

Inter-filament coupling loss Following the work of A. Verweij the time constant $\tau_{i f c l}$ for the inter-filament coupling loss is calculated by [59]:

$$
\tau_{i f c l}=\frac{\mu_{0}}{2 \rho_{e f f}}\left(\frac{L_{p, f}}{2 \pi}\right)^{2},
$$

where $\rho_{\text {eff }}$ is the effective resistivity of the strand matrix and $L_{p, f}$ is the filament twist pitch. This results in a time constant of

$$
\tau_{i f c l} \approx 26 \mathrm{~ms} .
$$

Now the inter-filament coupling loss can be calculated with:

$$
\dot{Q}_{i f c l}=\frac{2 \pi \tau_{i f c l} \omega B^{2}}{\mu_{0}\left(1+\omega^{2} \tau_{i f c l}^{2}\right)} f,
$$


where $\omega=2 \pi f$. Table 3.5 lists the inter-filament coupling loss for the different heating system setting. They range from $0.49 \mathrm{~mW} \mathrm{~cm}^{-3}$ to $3.23 \mathrm{~mW} \mathrm{~cm}^{-3}$.

Table 3.5. The inter-filament coupling loss calculated with Equation 3.9 for the different heating system circuit impedances.

\begin{tabular}{c|c} 
Impedance in $\Omega$ & Inter-filament coupling loss in $\mathrm{mW} \mathrm{cm}^{-3}$ \\
\hline \hline 157 & 3.23 \\
165 & 2.92 \\
178 & 2.52 \\
201 & 1.99 \\
235 & 1.45 \\
295 & 0.92 \\
339 & 0.70 \\
407 & 0.49
\end{tabular}

Cable Coupling Losses A. Campbell calculates the losses for a cable that arise from the contact resistance between the strands as follows [57]:

$$
\dot{Q}_{\text {cable }}=\frac{B^{2}}{4 \pi^{2}} \frac{\left(L_{p, f}-L_{p, s}\right)^{2}}{R} a,
$$

where $L_{p, f}$ and $L_{p, s}$ are the filament twist pitch and the strand twist pitch, respectively, and $R$ is the contact resistance between the strands. $a$ is a constant with the value $10^{-3} \mathrm{~s}^{-1} \mathrm{~m}^{-2}$. The cable coupling loss has a time constant that is calculated as following [59]:

$$
\tau_{\text {cable }}=1.65 \cdot 10^{-8} L_{p, s} \frac{N^{2}-4 N}{R} \approx 500 \mathrm{~ms},
$$

where $N$ is the number of strands.

The losses that result from the combination of several strands into one cable are listed in Table 3.6 for the different heating system impedances. They range from $0.15 \mathrm{~mW} \mathrm{~cm}^{-3}$ to $1.01 \mathrm{~mW} \mathrm{~cm}^{-3}$.

The inter-strand contact resistance is the parameter associated with the highest uncertainty in this calculation and the knowledge of this resistance is necessary to get a reliable result. Different groups measured the contact resistance between strands of the LHC type 01 superconducting cable. The design requirements for the contact resistance listed in the LHC design report are $15 \mu \Omega$ with a minimum of $10 \mu \Omega$ [4]. Richter et al. investigated the inter-strand contact resistance for different types of strand coatings and found for the LHC type 01 cable values of $1.7 \mu \Omega$ to $30 \mu \Omega$ for the cable without heat treatment 60 . After the heat treatment the inter-strand contact resistances were found to be lower. The value of $2.5 \mu \Omega$ for the interstrand resistance, as it is used for the calculation of the heating power, is in the range of the experimentally found value for the interstrand resistance, but 
Table 3.6. The cable coupling loss calculated with Equation 3.10 for the different heating system powers at a contact resistance of $2.5 \mu \Omega$

\begin{tabular}{c|c} 
Impedance in $\Omega$ & Cable coupling loss in $\mathrm{mW} \mathrm{cm}^{-3}$ \\
\hline \hline 157 & 1.01 \\
165 & 0.91 \\
178 & 0.79 \\
201 & 0.62 \\
235 & 0.45 \\
295 & 0.29 \\
339 & 0.22 \\
407 & 0.15
\end{tabular}

Table 3.7. Overview of the total loss in the sample.

\begin{tabular}{c|c} 
Impedance in $\Omega$ & Total AC loss in $\mathrm{mW} \mathrm{cm}^{-3}$ \\
\hline \hline 157 & 4.51 \\
165 & 4.06 \\
178 & 3.48 \\
201 & 2.73 \\
235 & 1.97 \\
295 & 1.24 \\
339 & 0.94 \\
407 & 0.65
\end{tabular}

lower than the nominal design value.

\section{Alternating current loss summary}

As a conclusion from the previous discussion Table 3.7 lists the total loss in the sample as a function of the heating system impedance. To get an impression on the contribution of the different loss mechanisms. Figure 3.7 shows the different $\mathrm{AC}$ loss components and the sum of the AC loss. The inter-filament coupling loss is the main contributor to the total loss in the cable, whereas the hysteresis loss is an order of magnitude lower than the other two loss mechanisms and contribute the least to the total loss. The cable coupling loss is between the hysteresis loss and inter-filament coupling loss, contributing about $20 \%$ to the total loss.

Taking into account the different time constants compared to the applied magnetic field frequency one can also explain why the main contribution of the loss is from the inter-filament coupling loss. For an applied magnetic field with a frequency of $50 \mathrm{~Hz}$, the inter-filament loss has a time constant of $26 \mathrm{~ms}$ and the cable coupling loss has a time constant of $500 \mathrm{~ms}$. This indicates that the applied magnetic field 


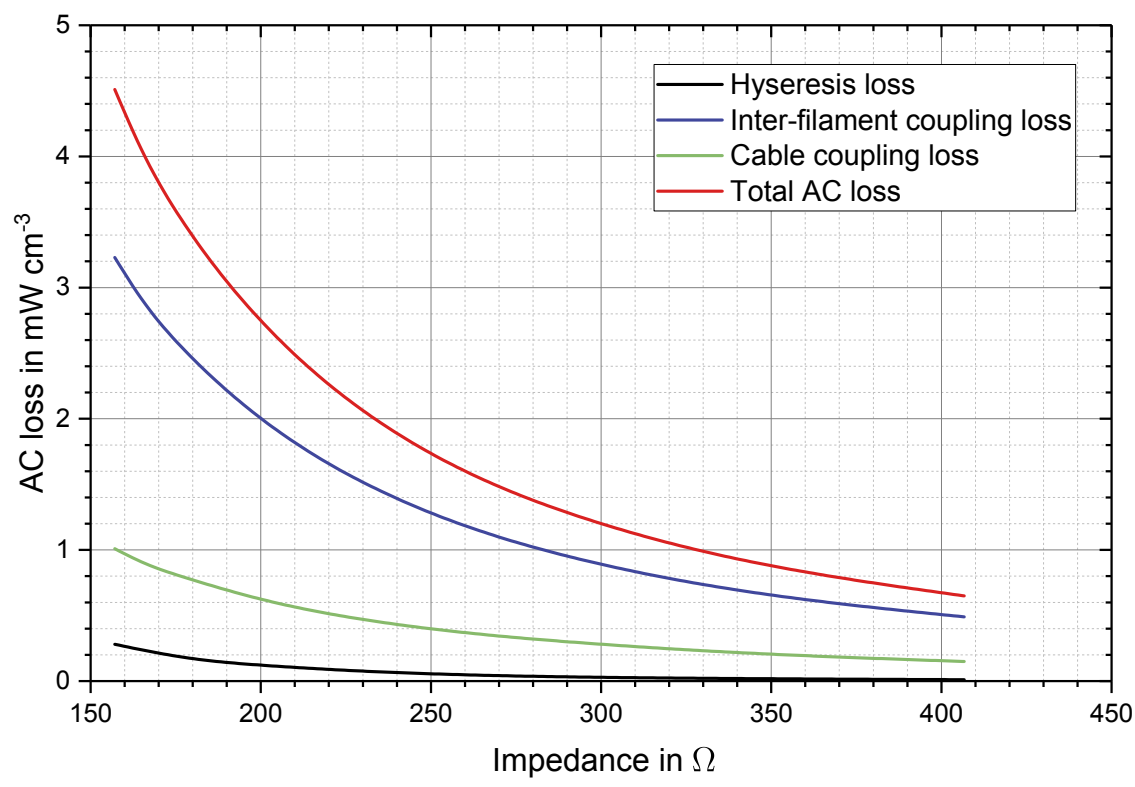

Figure 3.7. The contribution of the different AC loss mechanisms to the heat generation in a Rutherford cable stack subject to a $50 \mathrm{~Hz}$ magnetic field variation as a function of the heating system impedance.

couples strongly to the filaments in the strands and as a result the inter-filament loss contribute about $75 \%$ to the total loss. The small contribution of the hysteresis loss is not surprising when comparing the penetration magnetic field strength with the applied magnetic field strength. A quick calculation shows that the filaments are only penetrated to less than $10 \%$ at the lowest heating system impedance and the penetration percentage is decreasing with a decreasing outer magnetic field strength.

\subsection{Measurement Accuracy}

During an experiment different signals are measured. In the next section the different measured signals and the related accuracies are discussed in order to judge the quality of the experimental results.

\subsubsection{Absolute He Bath Temperature}

The base setting of each experiment is the He bath temperature. The He bath temperature is controlled by a MKS 651C pressure controller [61, which measures the cryostat pressure with an MKS $626 \mathrm{C}$ baratron absolute pressure gauge [62]. The controller throttles a MKS $653 \mathrm{~B}$ valve, which is mounted in the pumping 
connection in order to maintain the set point pressure 63. The baratron has an accuracy of $\pm 0.25 \%$ of reading. The pressure controller has a $0 \mathrm{~V}$ to $10 \mathrm{~V}$ linear analog output, which is digitised with a National Instruments 6225 measurement card 64. This combination of the measurement of the He bath pressure results in the maximum deviation for the absolute He bath temperature as shown in Table 3.8 The conversion from the saturation pressure of the He bath to temperature is made with the ITS-90 temperature scale, which uses the saturation pressure of a helium as the definition of the temperature in the experimentally investigated temperature range.

Table 3.8. Absolute pressure measurement and the resulting absolute He bath temperature deviation for typical pressure values of the measurement.

\begin{tabular}{rr|rr}
$\begin{array}{r}\text { Pressure } \\
\text { in Pa }\end{array}$ & $\begin{array}{r}\text { Pressure error } \\
\text { in Pa }\end{array}$ & $\begin{array}{r}\text { Temperature } \\
\text { in K }\end{array}$ & $\begin{array}{r}\text { Temperature error } \\
\text { in } \mathrm{mK}\end{array}$ \\
\hline \hline 4142 & \pm 14 & 2.1 & \pm 1.2 \\
2300 & \pm 9 & 1.9 & \pm 1.2 \\
1128 & \pm 6 & 1.7 & \pm 1.4
\end{tabular}

\subsubsection{He Bath Temperature Stability}

The stability of the He bath temperature is estimated from the standard deviation of a separate Cernox ${ }^{\mathrm{TM}}$ placed in the He bath to be better than $\pm 0.26 \mathrm{mK}$ at $2.1 \mathrm{~K}$ and better than $\pm 0.7 \mathrm{mK}$ at a He bath temperature of $1.7 \mathrm{~K}$. The He bath temperature stability is better by more than one order of magnitude than the absolute He bath temperature based on stability of the He bath temperature sensor.

\subsubsection{Absolute Sample Temperature}

The experiment for a known He bath temperature is based on the measurement of two temperatures and the knowledge of the heating power during an experimental run. The He bath temperature and the sample temperature are measured with Cernox $^{T M}$ bare chip sensors. Each sensor is connected to a separate Keithley 2410 source meter [65] that supplies a stable DC measurement current of $3 \mu \mathrm{A}$ to the sensors and the voltage drop across each sensor is measured by a single Keithley 3706A multimeter [66] equipped with a Keithley 3724 switching card. At a temperature of $1.7 \mathrm{~K}$ the current deposits less than $100 \mathrm{nW}$ in the temperature sensor, the sensor self-heating is therefore minimal and the power can be neglected in comparison to the deposited power from the heating system. Table 3.9 gives the accuracy of the temperature measurement based on this set-up. 
Table 3.9. Absolute temperature accuracy for sample sensor A.

\begin{tabular}{cccll} 
Bath temperature & $\begin{array}{c}\text { Resistance } \\
\text { Sensitivity }\end{array}$ & $\begin{array}{l}\text { Resistance } \\
\text { error }\end{array}$ & $\begin{array}{l}\text { Temperature } \\
\text { error } \\
\text { in } \mathrm{mK}\end{array}$ \\
\hline \hline 2.1 & in $\Omega$ & in $\Omega \mathrm{K}^{-1}$ & in $\Omega$ & \pm 2.5 \\
1.9 & 4610 & 3372 & \pm 8 & \pm 2.0 \\
1.7 & 5405 & 4640 & \pm 9 & \pm 1.6
\end{tabular}

\subsubsection{Relative Sample Temperature}

For the interpretation of the measurement the relative temperature increase is of much higher relevance than the absolute temperature. For the data analysis the measurement data the sample sensor is referenced to itself. In this case the measurement accuracy of the absolute temperature is not an accurate description. Instead the standard deviation of the sensor in steady-state conditions is used as the measurement accuracy for an individual measurement. The standard deviation of the sensors are smaller than $10 \mu \mathrm{K}$ independent of the He bath conditions. This relative accuracy is of vital importance for the interpretation of the measurement and for the determination of the deposited heat during the calorimetric measurements.

With the heating system relying on an oscillating magnetic field a voltage can be induced in the temperature sensors. To estimate the error resulting from the induced voltages the voltage across the sensors was measured repeatedly without supplying any current to the sensors. During these measurements it was found that the induced voltages are smaller than $\pm 1 \mu \mathrm{V}$ resulting in an error of $\pm 50 \mu \mathrm{K}$.

\subsection{Data Analysis}

The data recorded during a measurement is analysed with the help of a relaxation model in order to extract two characteristic values for each measurement. The two values obtained from the relaxation model are the steady-state temperature difference and the characteristic time for the sample to reach steady-state.

Figure 3.8 shows a sketch of the model. It consists of the sample with a heat capacity $C$ at a temperature $T_{s}$ and the He bath that is kept at a constant temperature $T_{b}$. The thermal link between the sample and the He bath has a thermal resistance of $R$. A heat flux $\dot{Q}_{d i s s}$ is generated in the sample during the experiment and $C$ and $R$ are considered constant over the measured range.

Firstly a balance around the sample is made:

$$
-\frac{T_{s}-T_{b}}{R}+\dot{Q}_{d i s s}=C \frac{\mathrm{d}\left(T_{s}-T_{b}\right)}{\mathrm{d} t} .
$$

Then the full solution for Equation 3.12 is: 


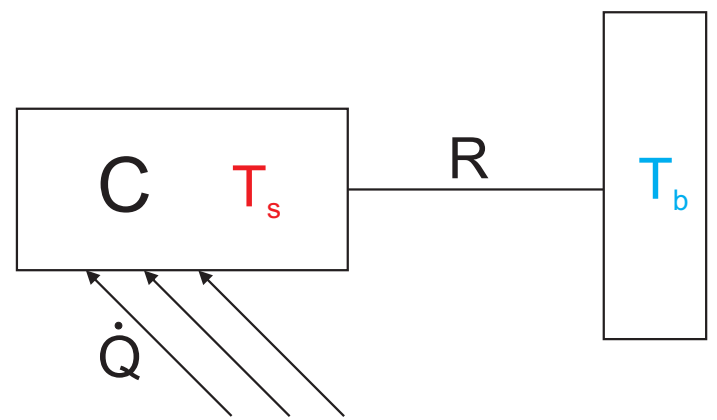

Figure 3.8. Schematic of the adiabatic set-up. The heat $\dot{Q}$ is dissipated in the sample with a heat capacity $C$. The sample has a thermal link to the cooling He bath with a resistance $R$. The temperature of the sample is $T_{s}(t)$, the He bath temperature is $T_{b}$.

$$
T_{s}-T_{b}=R \dot{Q}_{d i s s}\left(1-\exp \left(-\frac{t}{R C}\right)\right) .
$$

When applying the Equation $(3.13)$ to the measurement data two fit values are obtained. The steady-state temperature difference between the sample and the He bath and the characteristic time that describes the warm-up curve of the sample.

Taking equation Equation 3.13 the two extracted values are:

$$
\Delta T_{\max }=R \dot{Q}_{\text {diss }}
$$

and

$$
\tau=R C .
$$

such that Equation 3.13 reads as follows:

$$
\Delta T(t)=\Delta T_{\max }\left(1-\exp \left(-\frac{t}{\tau}\right)\right) .
$$

Figure 3.9 shows the measurement data and the fit using Equation (3.16). The fit quality is very good and the adjusted $\mathrm{R}^{2}$ for the fit is better than 0.98 .

A fit is made for each measurement individually. Since three measurements have been conducted for each condition the presented data is an average of these three individual fits. The deviation between the fit parameters for one experimental condition is smaller than $\pm 0.5 \mathrm{mK}$ for the steady-state temperature difference and smaller than $\pm 30 \mathrm{~ms}$ for the time constant. The standard deviation of the steadystate temperature difference is smaller than the He bath temperature stability and consequently the He bath stability is used in the measurement result discussion. 


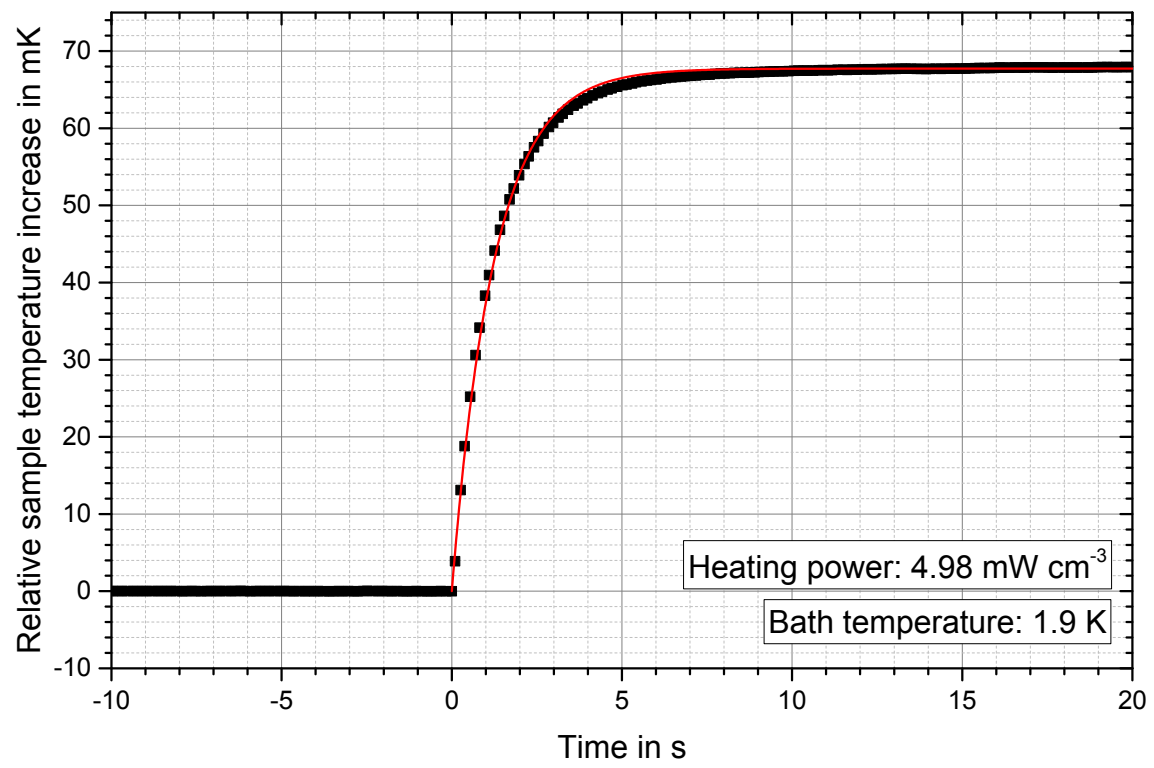

Figure 3.9. Example of a sample warm-up curve for a heating power of $4.98 \mathrm{~mW} \mathrm{~cm}^{-3}$ at a He bath temperature of $1.9 \mathrm{~K}$. The red line is a fit with the Equation (3.16), where $\Delta T$ is $68.7 \mathrm{mK}$ and $\tau$ is $1.20 \mathrm{~s}$. 


\section{Calorimetry}

Considering the strong dependency of the $\mathrm{AC}$ loss on the inter-strand contact resistance and the related uncertainty of its value, it was decided to measure the deposited heat calorimetrically. In this chapter the calorimetric measurements is described in detail and the results of two different measurement methods used to determine the amount of heat generated in the sample are reported. The first method relies on the knowledge of the sample properties such as density and specific heat whereas the second method relies on the knowledge of the set-up characteristics.

\subsection{Adiabatic Method}

The adiabatic method to measure the amount of deposited heat is similar to a heat capacity measurement. The sample is placed in an adiabatic environment where it is decoupled from the environment and the thermal contact with the environment is minimised as much as possible. Then the experiment is conducted and the temperature increase of the sample is used together with the known heat capacity of the sample to determine the heating power per unit volume following:

$$
\frac{\dot{Q}}{V}=\rho c_{p} \frac{\mathrm{d} T}{\mathrm{~d} t} .
$$

\subsubsection{Measurement Principle}

As explained very briefly in the preface of this section the adiabatic measurement relies on an as complete as possible decoupling of the sample from the environment. In such a case the temperature increase of the sample is a direct indicator for the amount of heat deposited in the sample. This requires precise knowledge of the material properties such as the temperature dependent heat capacity at constant pressure $c_{p}$ and the density $\rho$ of the sample. For a composite sample as it is investigated in this work also the composition of the sample needs to be known.

The equation used in the analysis of the adiabatic calorimetry data is the same equation that is used for the data analysis of a classic measurement. This is possible since the idea behind the measurement and the calorimetry is similar. The big difference between the two is the thermal link. In the case of the normal measurement the sample is immersed in He II and thus has a very good thermal link. In the case of the calorimetry the sample needs to be placed in a vacuum environment to decouple the sample from the environment.

For the cool-down a He atmosphere of 1 mbar is maintained in the fibre-glass reinforced vessel to increase the cool-down speed. After the cool-down the thermal 
link is minimised as much as possible by evacuating the fibre-glass reinforced epoxy vessel to a pressure lower than $3 \times 10^{-4}$ mbar. This reduces the thermal connection to the support structure of the sample holder.

Equation 3.13 requires knowledge of the thermal link $R$ of the sample to the He bath in order to calculate the amount of deposited heat $\dot{Q}_{\text {diss }}$ an information that is difficult to obtain without proper knowledge of the heating power. The thermal resistance is still non-negligible even though it has been reduced as much as possible.

To circumvent the unknown thermal link the time derivative of Equation 3.13 is used. The time derivative of Equation $\sqrt{3.13}$ is as follows:

$$
\frac{\mathrm{d}\left(T_{s}-T_{b}\right)}{\mathrm{d} t}=\frac{\dot{Q}_{d i s s}}{C} \exp \left(-\frac{t}{R C}\right) .
$$

Taking now the limit at $t=0$ gives us the final expression:

$$
\left.\frac{\mathrm{d}\left(T_{s}-T_{b}\right)}{\mathrm{d} t}\right|_{t=0}=\frac{\dot{Q}_{d i s s}}{C} .
$$

With this expression the amount of deposited heat $\dot{Q}_{\text {diss }}$ can be calculated from the heat capacity of the sample and the initial temperature slope. No further knowledge on the thermal link $R$ is necessary to calculate the amount of deposited heat.

\subsubsection{Accuracy Estimation}

The adiabatic calorimetry requires precise knowledge of the absolute temperature of the sample and the sample composition for the calculation of the material properties.

The temperature sensor in the sample that in an experiment is immersed in He II has in the case of the calorimetry a higher error than during an experiment in He II. This is due to the fact that in an experiment in He II the temperature sensor can be calibrated in-situ and very precisely with respect to the saturation temperature of the He bath. The temperature sensor in the case of the adiabatic calorimetry is placed in vacuum and the temperatures that are reached are much higher than in an experiment in He II. The calibration curve for the temperature sensor in this elevated temperature range is extrapolated and the error for this extrapolation is estimated to be $100 \mathrm{mK}$ up to a temperature of $4 \mathrm{~K}$. Since only the first slope of the temperature curve is used for the calculation this temperature range is considered to be sufficiently wide. The increased error is due to the extrapolation of the calibration curve and due to the sensor self-heating.

The second main error source for the adiabatic calorimetry is the material composition. Based on the specifications of the LHC superconducting strand the errors for the heat capacity and the density are considered. The copper to superconductor ratio for the strand is specified to be in the range 1.6 to 1.7 and the composition of the superconductor is specified to be between 46 to 48 weight- $\%$ of titanium content. 
All in all this uncertainty in the material composition together with the error of the absolute temperature results in an error of up to $20 \%$ on the determined heating power.

An additional error results from the fit that is necessary to determine the initial slope of the warm-up curve. This error results from the unknown exact starting time of the heating cycle and the time resolution of the temperature measurement of $10 \mathrm{~ms}$. This error is highest for the highest heating power and gets smaller with a decreasing heating power. The variation of the fit parameters such that a confidence level of $95 \%$ is reached is used to determine this error contribution. This error is by far the highest contribution to the error of the adiabatic calorimetry.

\subsection{Heat Meter Calorimetry}

The second method to determine the heating power is based on the idea of a heat meter. It uses an additional thermal barrier to create a heat flux dependent temperature gradient, which is then used to determine the heating power.

\subsubsection{Measurement Principle}

A heat meter is a known way to determine a heat flux. In a heat meter an additional thermal resistance is placed in the path of the heat flux thus creating an additional small temperature gradient, which is proportional to the heat flux. To calibrate the heat meter a known heat load is deposited in the sample and the temperature difference is measured. This results in a mathematical expression for the relation between the heating power and the temperature difference across the additional thermal resistance.

In this set-up the fibre-glass reinforced epoxy insert used to create the conditions for pressurised superfluid helium can directly be used as a heat meter. The insert represents a thermal barrier and will cause the inner He bath temperature to increase during a measurement cycle. The inner He bath temperature increase is then proportional to the heating power. While the vessel is designed to limit the temperature increase to less than $2 \mathrm{mK}$ for the heating power range of interest, this temperature increase is still large enough to be measurable by the He bath temperature sensor.

To characterise the heat meter a small heater is installed in the insert. This small heater is used to deposit a known heating power such that together with the measured temperature difference between the primary and the secondary He bath the thermal characteristic of the heat meter can be determined.

In order to measure the heating power that is deposited in the sample by AC loss two measurement runs are necessary. In the first run the sample and the sample holder are placed in the insert and during a measurement cycle the He bath temperature in the insert is recorded. The He bath temperature increase in the fibre-glass reinforced epoxy vessel is proportional to the amount of deposited heat in the sample and the sample holder. This procedure is repeated for different outer He bath temperatures with all available heating power settings. 
After the measurement cycle the small heater is used to deposit a range of known heating power in the insert and the inner He bath temperature is again recorded. This serves as a calibration of the heat meter. The calibration is then used to calculated the heating power in the sample and the sample holder from the inner He bath temperature increase.

For the second measurement run the sample is removed from the insert and the now empty sample holder is mounted in the set-up and the same procedure as in the previous measurement run is followed. First a measurement cycle with the AC magnetic field is executed and the helium He bath temperature in the insert is measured. Then the small heater is used to confirm the previously obtained heat meter characteristic. From this measurement the heating power generated in the sample holder is determined.

For each possible combination of outer helium He bath temperature and heating system setting two measurements are available, one with the sample and another one without the sample. The heating power is then determined from the difference between two measurements at identical external conditions and in a last step the calculated heating power is divided by the sample volume in order to obtain the heating power per unit volume of sample. The total sample volume is:

$$
V_{\text {sample }}=4 \cdot 1.9 \mathrm{~mm} \cdot 15.1 \mathrm{~mm} \cdot 140 \mathrm{~mm}=16.1 \mathrm{~cm}^{3} .
$$

This volume contains the volume of the strands and the voids between the strands. The polyimide insulation is not included in this volume since it is a soft material compared to the strand material and will deform under compression, thus changing the volume. The voids inside the Rutherford cable are included in this volume in order to make the calculated heating power comparable with the quench threshold values calculated on the magnet scale.

\subsubsection{Heat Meter Calorimetry Error Discussion}

To characterise the heat meter a known amount of heat is deposited in the helium vessel and the temperature rise in the helium vessel is recorded while the outer He bath temperature is kept constant. The heating power of the small heater is measured with a precision of $0.3 \%$. Together with the relative He bath temperature increase the characteristic of the heat meter is then determined. To get an analytic functional dependency of the heating power on the relative helium vessel temperature increase a third degree polynomial is fitted to the measurement data for the three He bath temperature independently. The adjusted $\mathrm{R}^{2}$ of the three fit functions is better than 0.999 and it is concluded that the function describes the thermal behaviour of the helium vessel sufficiently well.

For each of the runs the heating power in the sample and the sample holder is calculated. The heating power error for each of the measurements is determined from the first derivative of the heat meter characteristic function with respect to the temperature. To obtain the power that is generated in the sample the difference between the two measurements is taken and the maximum error of the actually measured heating power is taken as the error for the sample heating power. To 


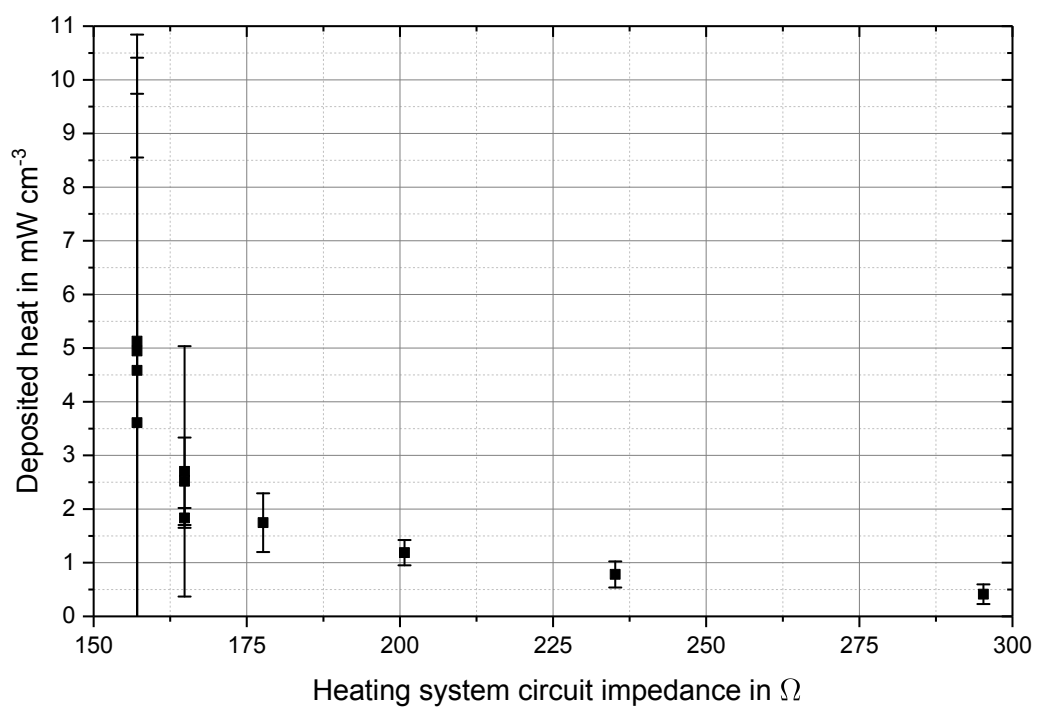

Figure 4.1. The deposited heat against the heating system circuit impedance.

obtain the heating power independent of the sample size the total deposited power is finally divided by the sample volume.

The volume of the sample is also associated with a measurement error. The error of the cable thickness according to the LHC design report is $(1.900 \pm 0.006) \mathrm{mm}$ and the allowed tolerance in the cable width is $(15.10 \pm 0.02) \mathrm{mm}$ [4. The sample length is $(140 \pm 2) \mathrm{mm}$. Together these errors amount to $0.08 \mathrm{~cm}^{3}$.

\subsection{Calorimetry Results}

\subsubsection{Adiabatic Method Results}

The specific heat of different compositions of Niobium-Titanium superconductors ranging in Niobium content of $41.23 \%, 46.49 \%, 50.39 \%$ and $52.80 \%$ in weight has been measured by Collings et al. where all four different alloy compositions show similar behaviour in their heat capacity 67. The heat capacity of the alloy with $52.80 \%$ Niobium is closest to the superconductor composition used in the sample and is used in this data analysis. The copper specific heat for the low temperature range can be found in 68. The result of Equation (4.3) applied to the measurement data is shown in Figure 4.1 graphically and listed in Table 4.1. The amount of deposited heat is highest at $5.46 \mathrm{~mW} \mathrm{~cm}^{-3}$ for a heating system circuit impedance of $158 \Omega$ and is decreasing in value to $0.42 \mathrm{~mW} \mathrm{~cm}^{-3}$ for a circuit impedance of $295 \Omega$. The calculated heating power at one heating system circuit impedance is the average of up to three measurements taken under the same conditions. The error bars are maximum error bars for one measurement condition. 
Table 4.1. The amount of deposited heat per volume of superconducting Rutherford cable for six different heating system circuit impedances calculated as a result of the adiabatic calorimetry.

\begin{tabular}{c|l} 
Impedance in $\Omega$ & $\begin{array}{l}\text { Generated heat } \\
\text { in } \mathrm{mW} \mathrm{cm}^{-3}\end{array}$ \\
\hline \hline 157 & $5.46 \pm 5.72$ \\
165 & $2.50 \pm 2.33$ \\
178 & $1.58 \pm 0.55$ \\
201 & $1.25 \pm 0.24$ \\
235 & $0.87 \pm 0.24$ \\
295 & $0.42 \pm 0.18$
\end{tabular}

The limited temporal accuracy of the temperature measurement and the fit quality for the initial slope lead to a considerable uncertainty of the calculated heating power for the low heating system circuit impedances. The uncertainty is of the same order of magnitude as the determined heating power itself. The uncertainty resulting from the steep initial temperature increase is reducing with lower heating powers.

\subsubsection{Heat Meter Calorimetry Results}

In a first step the thermal resistance posed by the fibreglass insert needs to be determined. To do this a known amount of heat is generated with a small cartridge heater that is placed inside the fibreglass insert and the temperature inside the insert is recorded with a Cernox ${ }^{T M}$ resistance thermometer. During the experiment the temperature of the outer He bath is kept constant with the help of a pressure regulator in such a way that the He bath temperature variation is kept below $15 \mu \mathrm{K}$. In Figure 4.2 the relative temperature increase of the inner $\mathrm{He}$ bath is plotted as a function of the deposited heat in the heater for three different outer He bath temperatures of $1.7 \mathrm{~K}, 1.9 \mathrm{~K}$ and $2.1 \mathrm{~K}$. The three curves all show an increase in the temperature increase for an increasing heating power. The three curves are not showing the same behaviour though, which is due to the changes in the effective thermal conductivity of He II with a changing He bath temperature. The relative temperature increase shows a cubic dependency on the deposited heat. Due to the dependency of the heat transfer in He II on the temperature three fit curves are obtained for the heat meter calibration, one for each He bath temperature.

During a measurement run the relative inner He bath temperature increase is recorded. The relative inner He bath temperature increase for the highest heating power at $1.7 \mathrm{~K}$ is $1.2 \mathrm{mK}$ and reaches down to a relative temperature increase of $0.1 \mathrm{mK}$ for a He bath temperature of $2.1 \mathrm{~K}$. The temperature increase is due to the heat load that is deposited in the sample and the other metallic components of the set-up and a reference measurement is needed to compensate for the heat that is generated in the other components of the set-up. 


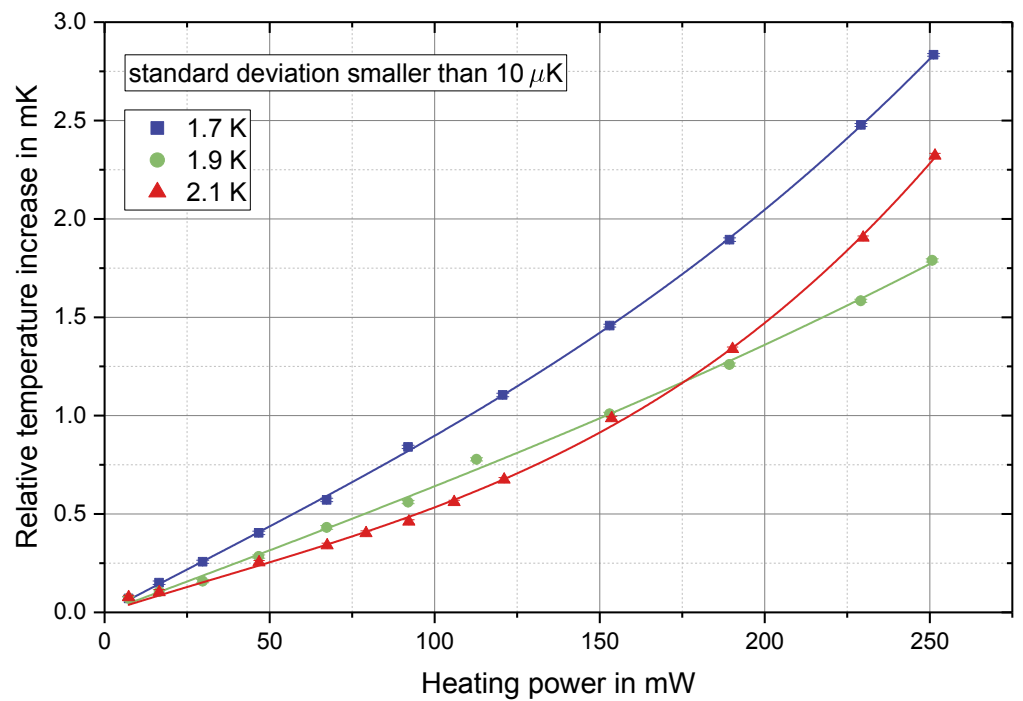

Figure 4.2. The relative inner He bath temperature increase as a function of the deposited heat for outer He bath temperatures of $1.7 \mathrm{~K}, 1.9 \mathrm{~K}$ and $2.1 \mathrm{~K}$. The lines are third order polynomial fit curves. The standard deviation of the temperature measurement is smaller than $10 \mu \mathrm{K}$.

A reference measurement of the set-up without the sample is performed in a second step in order to compensate for the heat deposited in other metallic components of the set-up. At a He bath temperature of $1.7 \mathrm{~K}$ the relative inner $\mathrm{He}$ bath temperature increase reaches $0.41 \mathrm{mK}$ for the smallest heating system circuit impedance and thus the highest possible heating.

The deposited power during the experiments with and without sample is determined in the next step. The difference between the two is the heat that is deposited in the sample. In order to make the comparison between the theoretical calculation and the adiabatic calorimetry this deposited heat is then divided by the sample volume.

The amount of heat that is deposited in the sample per unit volume as a function of the heating system circuit impedance is calculated for three different outer He bath temperatures $1.7 \mathrm{~K}, 1.9 \mathrm{~K}$ and $2.1 \mathrm{~K}$. The result of these calculations are tabulated in Table 4.2 . They range from $5.04 \mathrm{~mW} \mathrm{~cm}^{-3}$ for a circuit impedance of $157 \Omega$ to $0.79 \mathrm{~mW} \mathrm{~cm}^{-3}$ for a circuit impedance of $295 \Omega$.

In Figure 4.3 the data from Table 4.2 is plotted and within the error margin a hyperbolic fit is placed through the data points for all He bath temperatures. The fit function Equation 4.5 is then used for the calculation of the deposited heat in the analysis of the experimental data.

$$
\frac{\dot{Q}}{V}=\frac{1}{a+b Z}
$$


Table 4.2. The amount of in the sample deposited heat per volume for three different He bath temperatures and for six different heating system circuit impedances.

\begin{tabular}{c|lll} 
Impedance & $\begin{array}{l}\text { Sample power at } \\
1.7 \mathrm{~K}\end{array}$ & $\begin{array}{l}\text { Sample power at } \\
1.9 \mathrm{~K}\end{array}$ & $\begin{array}{l}\text { Sample power at } \\
\text { in } \mathrm{mW} \mathrm{cm}^{-3}\end{array}$ \\
in $\Omega$ & in $\mathrm{mW} \mathrm{cm}^{-3}$ & $\mathrm{~mW} \mathrm{~cm}^{-3}$ \\
\hline \hline 157 & $5.00 \pm 0.12$ & $5.04 \pm 0.16$ & $4.58 \pm 0.18$ \\
165 & $4.15 \pm 0.12$ & $4.03 \pm 0.16$ & $4.36 \pm 0.17$ \\
178 & $3.34 \pm 0.12$ & $3.03 \pm 0.15$ & $3.36 \pm 0.17$ \\
201 & $2.31 \pm 0.11$ & $1.95 \pm 0.15$ & $2.26 \pm 0.16$ \\
235 & $1.63 \pm 0.11$ & $1.25 \pm 0.14$ & $1.60 \pm 0.16$ \\
295 & $0.79 \pm 0.10$ & $0.81 \pm 0.14$ & $1.09 \pm 0.16$
\end{tabular}

where $a$ is a constant equal to $-700.45 \times 10^{-3} \mathrm{~cm}^{3} \mathrm{~mW}^{-1}, b$ a second constant equal to $5.74 \times 10^{-3} \mathrm{~cm}^{3} \mathrm{~mW}^{-1} \Omega^{-1}$ and $Z$ is the heating system circuit impedance in $\Omega$, which is the sum of the real part of the impedance of the $\mathrm{AC}$ magnetic field source at $50 \mathrm{~Hz}$ and the resistance of the in series resistor, which is used to vary the heating power.

\subsubsection{Calorimetry Summary}

Both methods to determine the heat load in the superconducting Rutherford cable stack show the same qualitative behaviour. By comparing the two methods it is clearly visible that the two methods have different accuracies, with the heat meter method having quasi constant errors over the whole measurement range compared to the adiabatic method where the error bars are high at the high heating powers and are decreasing with a decreasing heating power. The differences mostly arise from the way the values necessary for the calculation of the deposited heat are extracted from the recorded data. For the heat meter method the measured values can be immediately used for the calculation compared to the adiabatic method, where first an additional fit needs to be done. This additional fit increases the uncertainty of the calculated heating power due to the fast rising of the sample temperature. The highest temperature rise is in the order of $20 \mathrm{~K} \mathrm{~s}^{-1}$ and the limited sampling rate of $50 \mathrm{~Hz}$ therefore limits the precision with which the initial slope can be determined.

Both methods also have different dependencies of the measurement conditions. The adiabatic method is relying on the knowledge of the absolute temperature in contrast to the heat meter method, which is relying on the relative temperature increase. The relative temperature increase is known with a much higher precision, which together with the well known heat meter characteristic leads to an accurate heat load determination. For the adiabatic method the temperature measurement is much less accurate leading together with the limited temporal resolution of the sample temperature to an increased uncertainty of the determined heat load 


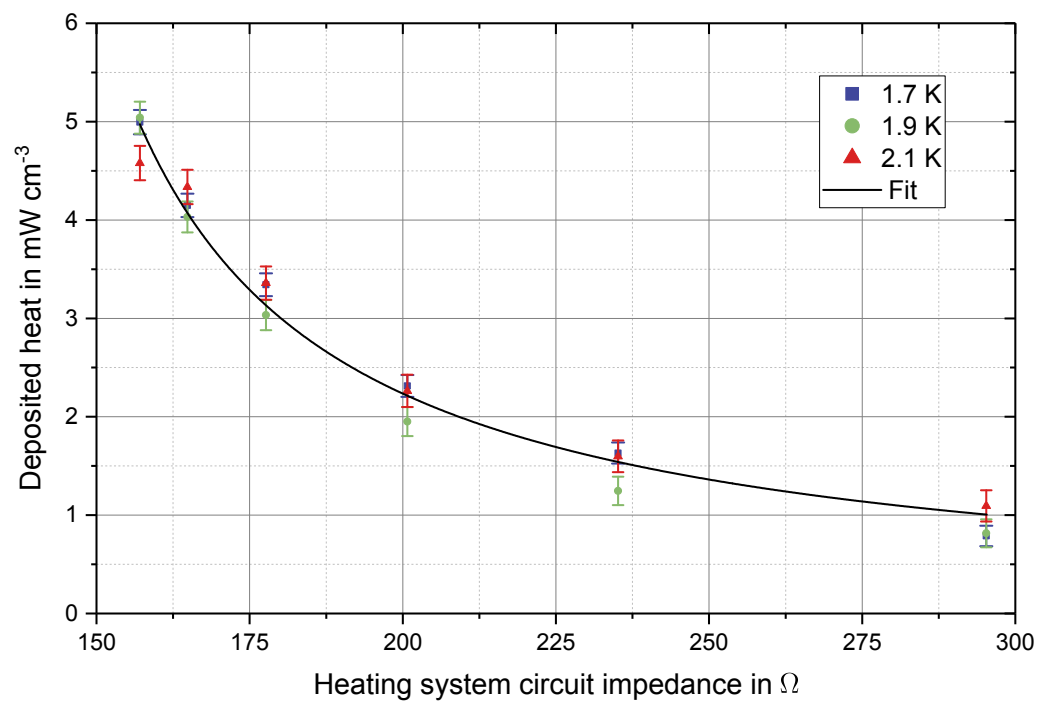

Figure 4.3. The amount of generated heat as a function of the circuit impedance of the heating system. The black line is a hyperbolic fit function, which describes the system very well within the error margin of the measurement points.

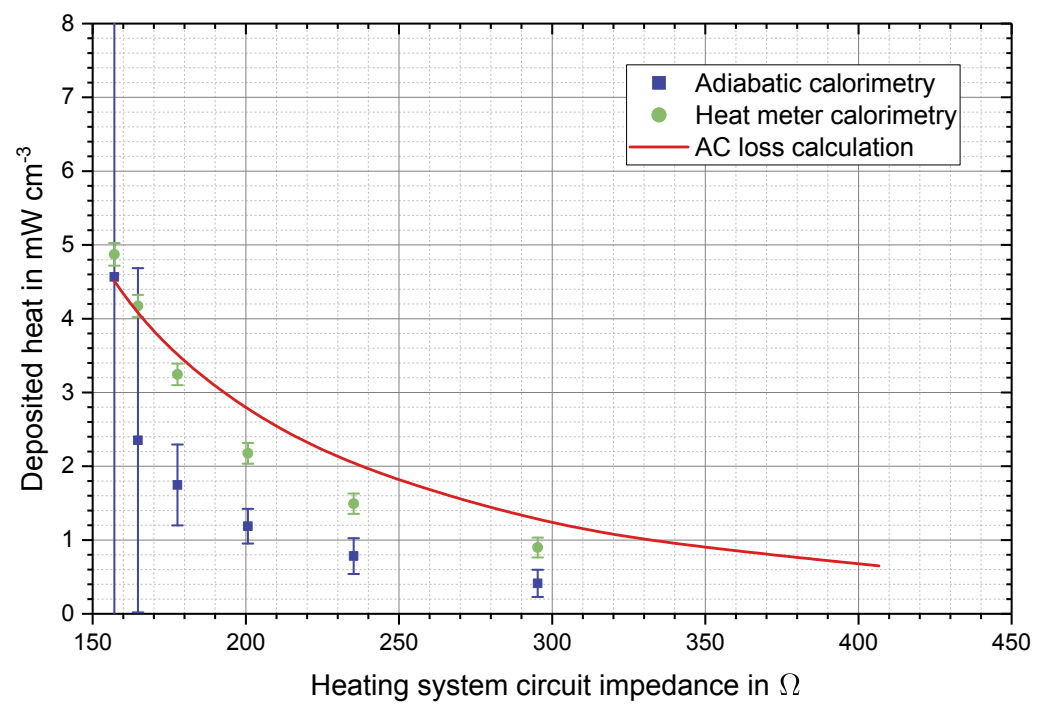

Figure 4.4. Comparison of the theoretical prediction (line) and the measured values (points) for the generated heat load in the superconducting Rutherford cable stack. 


\section{Calorimetry}

compared to the heat load determined by the heat meter method. It was thus decided to use the heat meter results for the data analysis of the experimental data.

A comparison between the calorimetric heat meter results with the theoretical prediction from the set-up description is shown in Figure 4.4. The theoretical calculation and the calibration results show qualitative agreement and also give the same order of magnitude results. For the measured range the theoretical prediction with an inter-strand resistance of $2.5 \mu \Omega$ gives a qualitatively similar behaviour as the heat loads determined by the calorimetric measurements. 


\section{Heat Transfer Model}

The aim of modelling is to increase the overall understanding of the thermal processes that take place in the studied sample. In this chapter a heat transfer model for the thermal processes in the investigated sample geometry is presented and it is compared to the vortex line density model discussed in Section 2.3.1. The complex geometry of the sample is simplified to establish a relatively easy model that is still capable of representing the thermal processes involved in the cooling of the sample and sample geometry parameters are introduced in the heat transfer model to fit the measurement data using known material properties. To model the thermal processes that take place in the cooling of the sample a direct transition model for the effective thermal conductivity of He II is presented, which is then used to calculate the effective thermal conductivity of He II in the laminar regime and in the turbulent regime. In the presented model the effective thermal conductivity of He II in a channel is always calculated assuming a round channel.

\subsection{Direct Transition Model for the Effective Thermal Conductivity}

In order to arrive at a full effective thermal conductivity model of He II it is essential to include the transition between the heat transfer regimes of He II. Since the parameters for the approach using a quantum Reynolds number to model the transition between the laminar regime and the turbulent regime are still very limited and are not applicable in the temperature range investigated in this work a direct approach to model the heat transfer regime transition is used.

For a given diameter $d$ a critical temperature gradient $\left(\frac{\Delta T}{l}\right)_{\text {crit }}$ can be calculated at which the effective thermal conductivity of the laminar regime is equal to the effective thermal conductivity of the turbulent regime. Using the cross-over between the functions of the heat flux densities in the laminar regime (see Equation (2.30) and the turbulent regime (see Equation (2.35) ) as a critical condition results in the following expression for the critical temperature gradient:

$$
\begin{aligned}
\dot{q}_{t u r b} & =\dot{q}_{l a m} \\
\left(\frac{f^{-1}}{\left(\frac{\Delta T}{l}\right)^{2.4}}\right)^{\frac{1}{3 \cdot 4}} \frac{\Delta T}{l} & =\frac{d^{2}(\rho s)^{2} T}{\beta \mu_{n}} \frac{\Delta T}{l} .
\end{aligned}
$$

And finally solved for the critical temperature gradient 


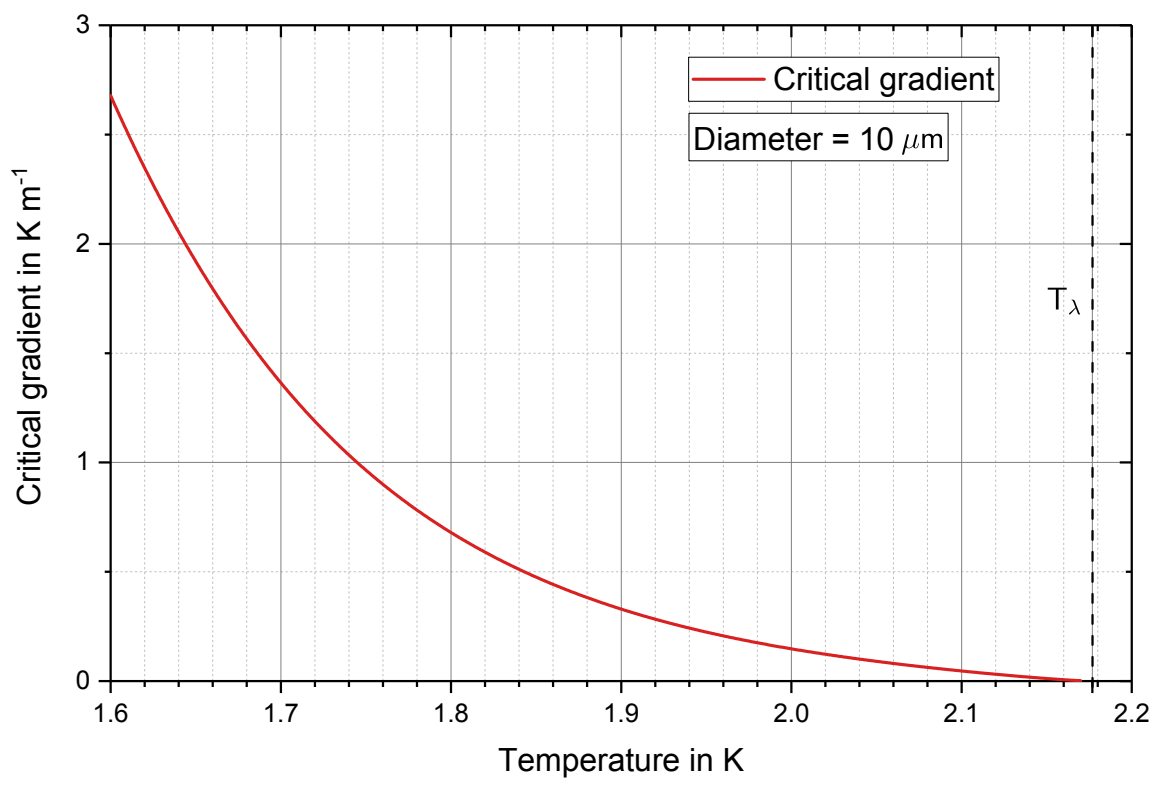

Figure 5.1. The critical temperature gradient as a function of temperature as calculated by the direct transition model for a round channel with a diameter of $10 \mu \mathrm{m}$.

$$
\left(\frac{\Delta T}{l}\right)_{\text {crit }}=\left(\frac{f^{-1}}{\left(\frac{(d \rho s)^{2} T}{\beta \mu_{n}}\right)^{3.4}}\right)^{\frac{1}{2.4}}
$$

where $\left(\frac{\Delta T}{l}\right)_{\text {crit }}$ is the critical gradient, $f^{-1}$ is the turbulent effective thermal conductivity coefficient (see Figure 2.6), $d$ the channel diameter and $\beta$ a geometry dependent parameter, which is 32 for round channels, $T$ the temperature and $\rho, s$, and $\mu_{n}$ are the total density, the specific entropy and the normal fluid viscosity of He II, respectively.

Figure 5.1 shows a plot of the critical temperature gradient as a function of temperature for a channel diameter of $10 \mu \mathrm{m}$. The plot shows that at a He bath temperature of $1.7 \mathrm{~K}$ the critical temperature gradient is about $2 \frac{1}{2}$ times higher than the critical temperature gradient at $1.9 \mathrm{~K}$. This means that for an identical heat flux density a channel can still be in the laminar regime at $1.7 \mathrm{~K}$ whereas at a temperature of $1.9 \mathrm{~K}$ the same channel is already in the turbulent regime for the same temperature gradient.

Figure 5.2 shows the effective thermal conductivity of He II at a bath temperature of $1.9 \mathrm{~K}$ as a function of the local temperature gradient. Below the critical gradient heat transfer occurs in the laminar regime, which is independent of the temperature 


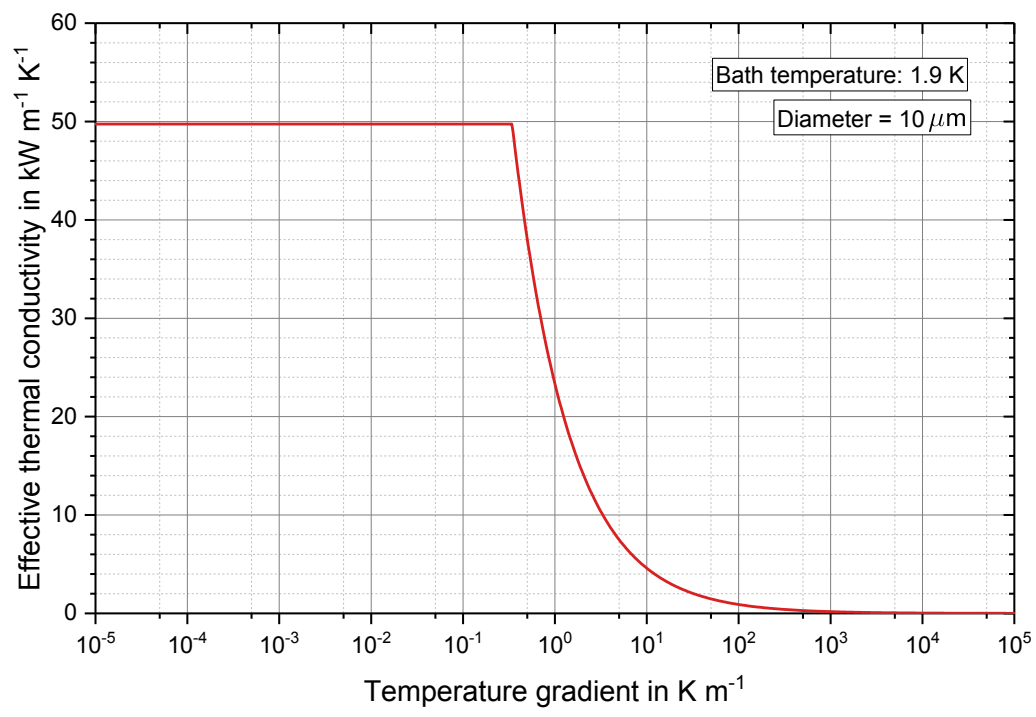

Figure 5.2. The effective thermal conductivity as a function of the temperature gradient for a channel diameter $10 \mu \mathrm{m}$ at $1.9 \mathrm{~K}$.

gradient. Once the critical gradient has been exceeded the transition occurs and heat transfer takes place in the turbulent phase, which is strongly dependent of the temperature gradient.

Figure 5.3 shows the combination of the previous two graphs Figure 5.1 and Figure 5.2 for the temperature range $1.45 \mathrm{~K}$ to $2.174 \mathrm{~K}$. It plots the effective thermal conductivity as a function of the temperature gradient and the bath temperature. The two regimes, the laminar regime and the turbulent regime, can clearly be distinguished from their different dependency on the temperature and the temperature gradient.

\subsubsection{Critical Velocity}

Calculating the effective thermal conductivity in the above-described way results in a straightforward way to calculate the transition between heat transfer regimes. As noted in section 2.3.1 about the vortex line density model the transition is a very complex process and is simplified in the way as represented here. The critical superfluid velocity for the transition predicted for zero-net mass flow conditions by the direct transition model is calculated using Equation 2.5) and Equation 2.6 as follows:

$$
v_{s c}=\frac{\rho_{n}}{\rho_{s}} \frac{\dot{q}_{c r i t}}{\rho s T} .
$$

Equation (5.3) together with Equation 2.30 predicts a dependency of the 


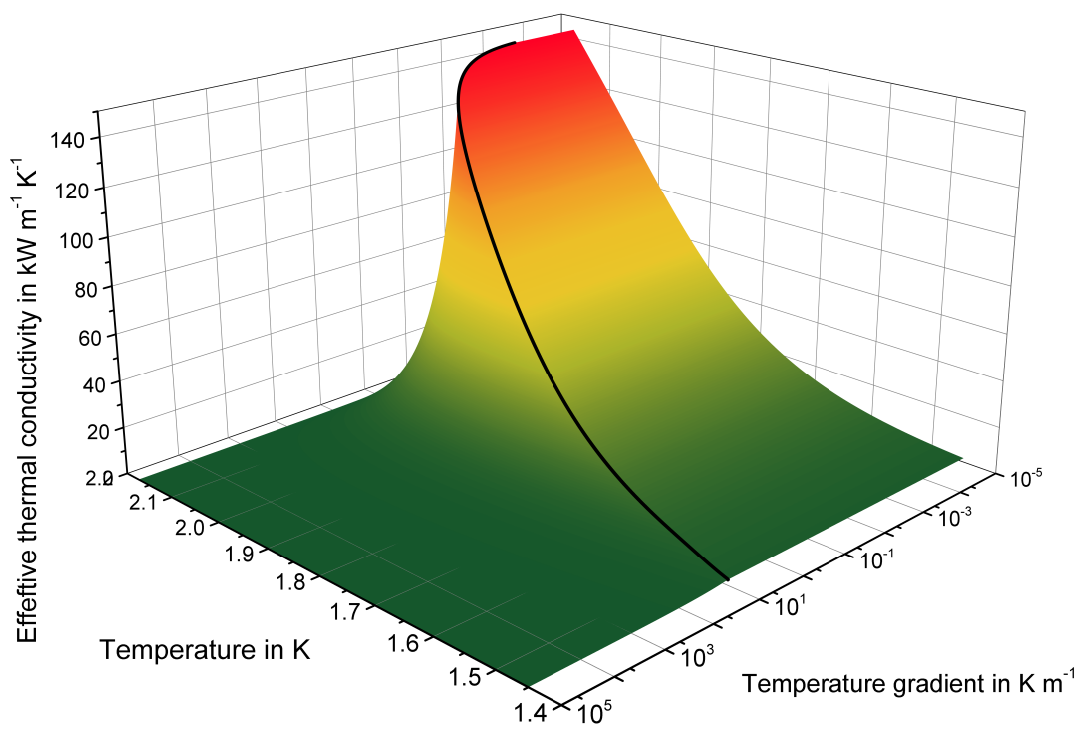

Figure 5.3. The effective thermal conductivity as a function of the temperature gradient and the temperature for a channel diameter of $10 \mu \mathrm{m}$. The black line indicates the critical gradient where the transition from the laminar to the turbulent regime occurs.

critical heat flux density, thus the critical superfluid velocity, of $\dot{q}_{c r i t} \propto d^{-0.83}$. This dependency can also be seen in Figure 5.4 where the critical superfluid velocity of the simplified direct transition model is compared with the critical superfluid velocity of two other models. The two other models shown in Figure 5.4 are the experimentally derived $d^{-\frac{1}{4}}$ model by van Alphen [69]:

$$
v_{s c} \simeq d^{-\frac{1}{4}} \text { in } \mathrm{cm} \mathrm{s}^{-1},
$$

where $d$ is in $\mathrm{cm}$ and the model derived by Feynman 30, which is a theoretical development for the critical superfluid velocity. Feynman derived the following expression for the transition between the heat transfer regimes:

$$
v_{s c}=\frac{h}{\pi m d}\left(\ln \frac{4 d}{a_{0}}-K^{\prime}\right),
$$

where $h$ is the Plank constant, $m$ is the mass of a helium atom, $a_{0} \simeq 1 \mathrm{~nm}$ the order of magnitude of a vortex core and $K^{\prime}$ a numerical constant.

All three models show an inverse size dependency and the qualitative agreement between the three models in Figure 5.4 is quite remarkable considering the different approaches to the critical superfluid velocity. 


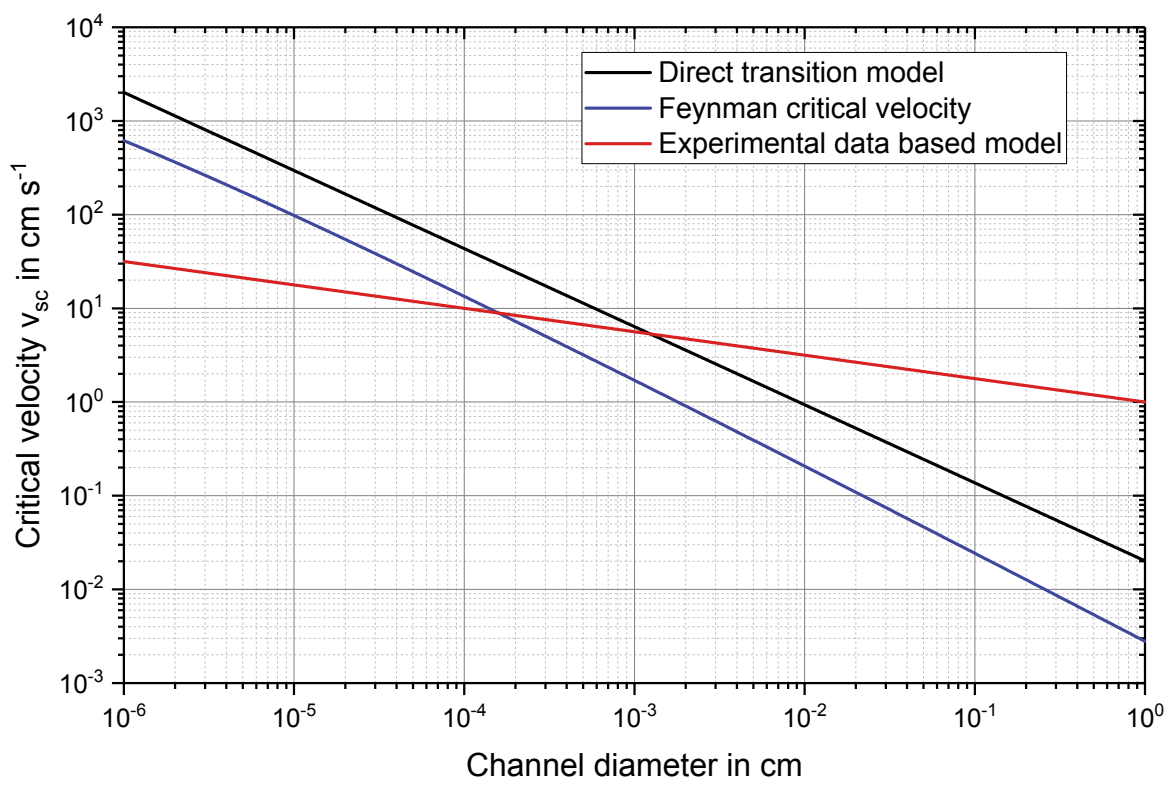

Figure 5.4. Comparison of different models for the critical superfluid velocity. Shown are the critical superfluid velocities calculated with Feynman's model [30], the experimental approach by van Alphen [69] and the presented direct transition model.

\subsubsection{Model Limitations}

It should be said that the application of the Gorter-Mellink law for fully developed mutual friction is only correct when the diameter of the investigated channel is in the order of $1 \mathrm{~mm}$ so that the laminar term can be neglected. For smaller diameters the correct description for the effective thermal conductivity should include the laminar term in addition to the mutual friction term [7]. In this work the turbulent regime is modelled only by the Gorter-Mellink law and the laminar term is neglected even though the channel diameters in the sample are considerably smaller than $1 \mathrm{~mm}$.

Another simplification made by the direct transition model is that it does not distinguish between the turbulent states TI and TII (compare Section 2.3.1). In case of the direct transition, the effective thermal conductivity changes directly to the fully turbulent regime where the Gorter-Mellink law applies and thus does not take into account the TI state as it is done in the vortex line density model of Sciacca et al 35. Figure 5.5 shows the effective thermal conductivity as it is calculated by the direct transition model compared to that calculated from the vortex line density model at a He bath temperature of $1.7 \mathrm{~K}$ and a channel diameter of $1 \mathrm{~mm}$. As can be seen the two models coincide for low heat fluxes, for higher heat fluxes only qualitative correspondence is achieved. This is due to the different 


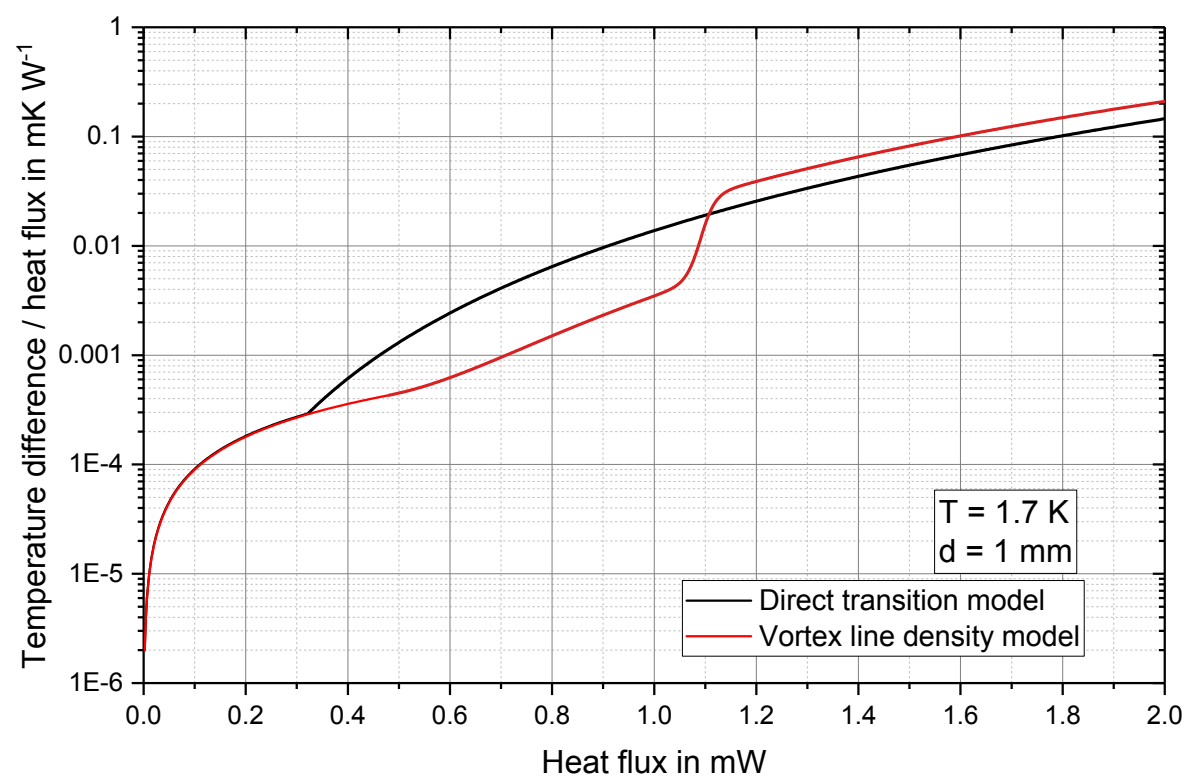

Figure 5.5. Comparison of the effective thermal resistance calculated with the vortex line density model and the direct transition model at $1.7 \mathrm{~K}$ for a channel diameter of $1 \mathrm{~mm}$ and a length of $100 \mathrm{~mm}$. The good agreement at low heat fluxes can be seen, the qualitative agreement at higher heat fluxes is also clearly visible. The deviation in the transition regime between the two models is also evident.

ways in which the fully turbulent TII regime is modelled. The laminar regime on the other hand is modelled in the same way thus the two models show identical behaviour. As expected the two models show a large deviation in the transition region.

The poor quantitative agreement between the two models at high heat fluxes can be related to the fact that Sciacca et al fitted the parameters of their model to measurement data from Martin et al [70] and thus achieved a very good agreement between their model and that set of measurement data. The direct transition model instead relies on a fit by Sato et al. 41 of the Gorter-Mellink mutual friction parameter $f^{-1}$ that is based on a much broader measurement data base and thus does not show perfect agreement with every single measurement but instead fits a much broader range of applications.

\subsection{Model Description}

As described in the introduction the studied sample geometry is very complex and needs to be simplified for the modelling. The sample consists of three components, 
the 28 solid strands that in the application carry the electric current, a dielectric polyimide insulation wrapping, consisting of three layers that insulate the cables electrically, and liquid He II that fills the voids between the strands, between the strands and the polyimide insulation and also between the three polyimide layers. The following subsections discuss each component of the sample separately and explains how it is implemented in the model.

\subsubsection{Model Composition}

\section{Superconducting cable and helium in the cable voids}

The strand temperature is taken to be uniform because of the high thermal conductivity of the strands and the high effective thermal conductivity of the He II in the voids between the strands. This assumption is justified as shown in the work of P.P. Granieri 52] who measured the thermal gradient that is created during a similar type of experiment inside the bulk of the superconducting sample. He showed that the temperature gradient inside the cable is small compared to the measured temperature difference between the cable and the outer He bath and can thus be neglected. The reason for this negligible temperature gradient inside the cable is the helium content in the cable voids. In a cable compressed with $50 \mathrm{MPa}$ the calculation of the helium content is straightforward taking the dimensions from 71]:

$$
\frac{V_{H e}}{V_{t o t}}=\frac{V_{t o t}-N_{\text {Strand }} V_{\text {Strand }}}{V_{\text {tot }}} .
$$

With the known cable dimensions, such as the twist pitch of the strand, the helium content is:

$\frac{V_{H e}}{V_{t o t}}=\frac{1.9 \mathrm{~mm} \times 15.1 \mathrm{~mm} \times 115 \mathrm{~mm}-28 \frac{\pi}{4}(1.065 \mathrm{~mm})^{2} 2 \sqrt{\left(\frac{115 \mathrm{~mm}}{2}\right)^{2}+15.1 \mathrm{~mm}^{2}}}{1.9 \mathrm{~mm} \times 15.1 \mathrm{~mm} \times 115 \mathrm{~mm}}$

$$
\frac{V_{H e}}{V_{t o t}} \approx 10 \% \text {. }
$$

Due to the deformation of the cable during the manufacturing and due to neglecting the edge, where the strand is changing the top layer of strands to the bottom layer, the void fraction has an estimated uncertainty of $\pm 2 \%$.

The $10 \%$ helium content together with the high effective thermal conductivity of the He II in the voids leads to the negligible small temperature gradient inside the cable [52] and thus the cable itself can be modelled as one cell with a uniform temperature.

\section{Unit cell}

For the purpose of the modelling, the sample is divided into multiple unit cells. A unit cell is a repetitive element found in the sample. It is $1.9 \mathrm{~mm}$ high, has a length 


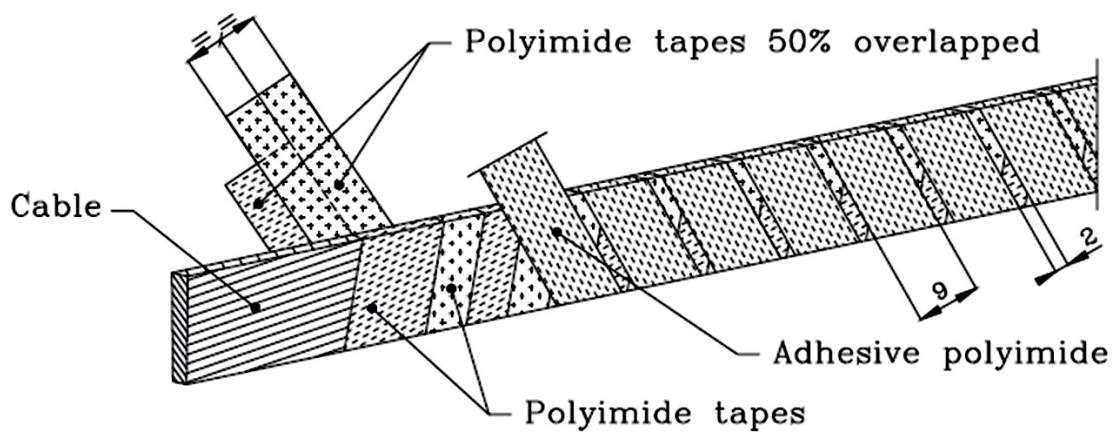

Figure 5.6. Illustration of the polyimide wrapping of the LHC superconducting cable CCERN [4].

of $7.55 \mathrm{~mm}$, which is half the cable width and is $4.11 \mathrm{~mm}$ wide. These dimensions result from the strand twist pitch divided by the number of strands in the cable. The unit cell has a volume of $58.9 \mathrm{~mm}^{3}$.

\section{Polyimide insulation of the Rutherford cable}

As a result of the negligible temperature gradient inside the cable the thermal barrier between cable and He bath is solely located in the polyimide insulation and the He II channels in the insulation. Therefore, it is necessary to look at the thermal properties of the insulating layer around the cable in detail.

Three polyimide layers are wrapped around the cable guaranteeing an electric insulation of the cable but also forming small channels between the layers, which are filled with He II. The inner two layers are formed by the same tape with a nominal thickness of $50 \mu \mathrm{m}$ and a width of $11 \mathrm{~mm}$. The inner tapes are wrapped with a $50 \%$ overlap in order to increase the protection of the cable against an electric break down. The third and outermost layer of the polyimide insulation is $69 \mathrm{\mu m}$ thick and has a width of $9 \mathrm{~mm}$. It is wrapped on top of the inner layers with a gap of $2 \mathrm{~mm}$ between turns. In this way the third layer creates wide artificial cooling channels that are filled with He II. Figure 5.6 illustrates the insulation wrapping.

This insulation pattern enables the cooling of the superconducting cable. Meuris et al. 47] found that the helium channels between the different layers play an essential role in the cooling and the insulation of the LHC magnet cable was chosen specifically to have a high helium permeability to improve the cooling provided by the helium.

The polyimide insulation itself will also contribute to the cooling of the cable via solid conduction. In the model the polyimide insulation is considered on the small face of the cable. The surface area that is taken into account is the total area of the small face for a unit cell. As the thickness of the insulation an average thickness is considered. Due to the three layer layout of the polyimide wrapping the shortest conduction length is $100 \mu \mathrm{m}$ and the thickest point of the insulation 
is $169 \mathrm{\mu m}$. The third layer covers nearly $\frac{9}{11}$ of the surface and thus an average thickness of the insulation of $157 \mu \mathrm{m}$ is used for the model.

The contribution of the wrapping on the broad face to the solid polyimide conduction is not taken into account because the cross section of this wrapping, which is transferring heat towards the He bath is much smaller than the surface area of the wrapping on the small face. At the same time the conduction length of the broad face is much longer than the thickness of the insulation wrapping on the small face, which also reduces the heat flux transported by the broad face. For example, if a conduction length of one quarter of the cable width is considered, then the ratio of heat flux for a He bath temperature of $1.9 \mathrm{~K}$ between the small and the broad face is:

$$
\frac{\dot{Q}_{\text {broad }}}{\dot{Q}_{\text {small }}}=\frac{A_{\text {broad }}}{A_{\text {small }}} \frac{l_{\text {small }}}{l_{\text {broad }}} \approx 0.3 \% .
$$

Less than $0.3 \%$ of the solid-conduction heat flux transported by the small face is transported through the broad face and it was thus decided to neglect the contribution of the polyimide insulation on the broad face in the model.

\section{Kapitza resistance}

The Kapitza resistance is an additional thermal contact resistance that occurs at the interface of liquid helium and solid materials. This resistance is increasing with decreasing temperature. Baudouy et al. measured the thermal conductivity of polyimide and the Kapitza resistance between polyimide and liquid helium in a temperature range relevant to the present study [72. From Figure 5.7 it can be seen that the polyimide wrapping on the small face is not in direct contact with the cable and the heat transferred through the polyimide is thus affected by the Kapitza resistance of liquid helium to polyimide. The buckling of the insulation is increasing when a higher compression force is exerted on the broad face of the cable. This causes the polyimide on the small face to be in contact with helium on both sides and therefore the Kapitza resistance needs taken into account twice, once on the inside of the wrapping and once on the outside where the wrapping is in contact with the He bath. The temperatures used to calculate the Kapitza resistance are the maximum sample temperature on the inside and the He bath temperature on the outside of the insulation.

An additional Kapitza resistance exists inside the cable where the heat that is deposited in the strands is transferred to the helium in the voids. This thermal resistance is not considered in the model since it is difficult to make a correct estimation of its value. This is also related to the uncertainty of the placement of the temperature sensor. It is unclear whether the measured temperature should be associated with the voids helium temperature or the cable temperature. For the purpose of the modelling it is assumed that the measured temperature is identical to the helium temperature in the voids and the Kapitza resistance does not need to be taken into account. 


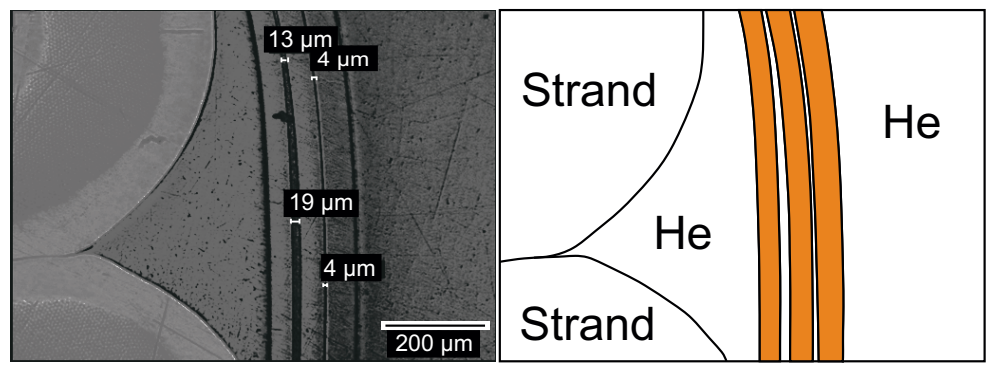

Figure 5.7. Microscope picture of the hand-wound polyimide insulation on the small face of the cable in the study of Granieri, [52, (left picture) and a schematic of the same picture on the right. The boundaries between different materials are shown, the polyimide insulation is coloured orange, the white spaces in the insulation are the helium cooling channels, the label He indicates the wide spaces that are filled with helium during an experiment. The channels in the insulation can be clearly seen in the schematic and the dimensions of these channels are given in the microscope picture.

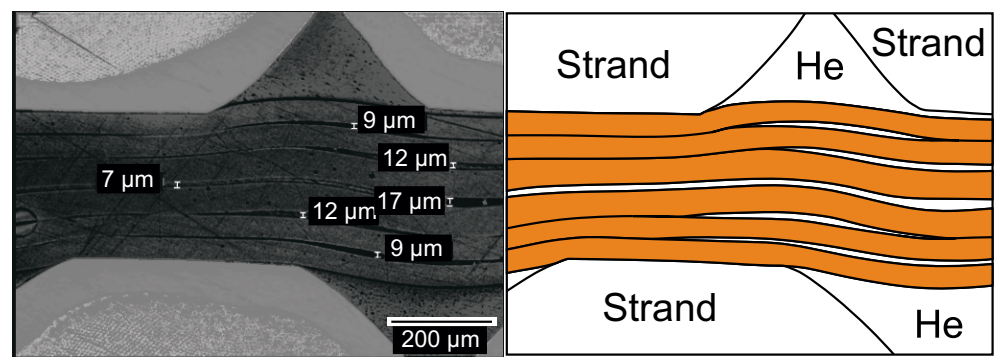

Figure 5.8. Microscope picture of the hand-wound polyimide insulation on the broad face in the study of Granieri, [52, (left picture) and a schematic of the same picture on the right. The polyimide insulation is coloured orange and the He cooling channels between the different layers can be clearly seen as the white gaps between the layers. The heights for some the created channels are given in the microscope picture on the left.

\section{Helium channels}

The inner two layers of the insulation form various channels. These channels present the main cooling connection for the cable and present direct connections between the He II in the voids in the cable and the He II bath. P.P. Granieri made cuts through different insulated cables to confirm the existence of these channels and measured their sizes [52]. Figure 5.7 and Figure 5.8 show microscope pictures of the insulation in the small face and the broad face of the cable, respectively.

At this point it should, however, be noted that the insulation of Granieri's cable samples were hand-wound, whereas the sample in the present study are taken from the actual cable production with machine-wound insulation. As a result the 


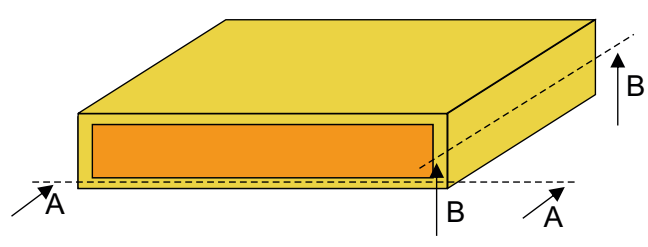

Small face channels

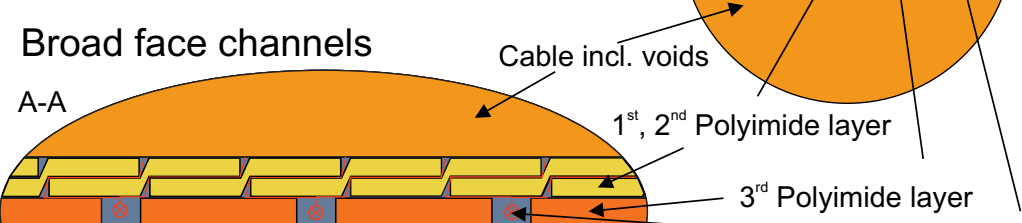

Symmetry axis

Helium

Figure 5.9. Illustration of the cooling channels in the insulation of a superconducting cable. The illustration shows the three different channels that connect the cable with the outer He bath. The cable is shown in orange, the inner two layers of the insulation in yellow and the third layer of the insulation in red. The helium in the insulation and the He bath is blue, with the transported heat flux indicated by the red arrows.

hand-wound insulation in Granieri's case is not as tight as the machine wound cable and the channel dimensions shown in Figures 5.7 and 5.8 are not representative for the the dimensions of the channels in the present study sample. Aside from the hydraulic diameter of the channels also the length of the channels is difficult to determine. In his work P.P. Granieri estimated the length of the channels by assuming that the channels would be always going in the most direct way [52. The channels in his work are thus always in a $90^{\circ}$ angle with respect to the axis along the surface of the insulation tape. This is certainly a start for the modelling of the heat transfer in the insulation but can also be considered too optimistic due to the irregularities in the insulation wrapping. From the pictures it can also be seen that the number of channels that is created depends on the location of the cut.

Considering all the uncertainties of the determination of the channel dimensions it was decided to make no assumption on the channel dimensions but to leave all geometric parameters such as number of channels, diameter and length as variables in the model.

In this work three channels are assumed to model the heat exchange of the cable to the He bath. At the small face a single channel is assumed that passes through the three polyimide layers, meandering out to the He bath (see Figure 5.9). This thermal path is named channel 1. At the broad face a path is assumed consisting effectively of two channels in series. First a narrow channel, named channel 2, passing the first two polyimide layers heading to the third layer, followed by a relatively wide channel (channel 3 ) through the $2 \mathrm{~mm}$ spacing of the third polyimide 


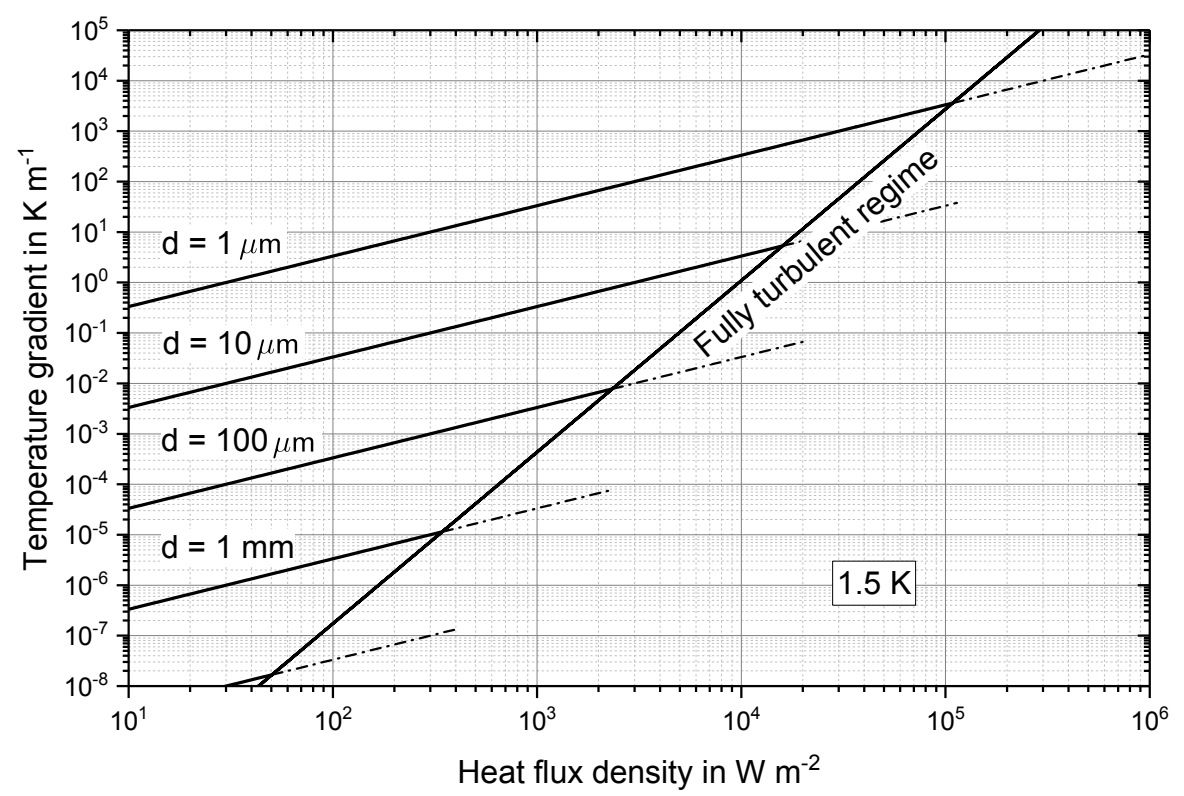

Figure 5.10. The temperature gradient as a function of heat flux density at $1.5 \mathrm{~K}$ as calculated by the direct transition model for different channel diameters. The laminar regime with its geometry dependency and the fully turbulent regime can clearly be seen. The dash-dotted line shows an extrapolation of the laminar regime. For the transition regime see Figure 5.5 .

layer leading to the He bath.

Each of these channels has a different geometry. One would expect that the channels 1 and 2 have about the same geometry as both of these are primarily formed by the same inner two polyimide layers. However, channel 2 is found on the broad face and thus is exposed to the compression force (see Figure 5.8). This leads to a reduced channel cross section and smaller equivalent diameter compared to the channel 1. This together with the fact that the combined length of channels 2 and 3 is longer than the length of channel 1 has an effect on the heat transfer regime that the different channels are experiencing. This is further clarified by Figure 5.10 where the temperature gradient is plotted as a function of the heat flux density for different channel diameters. In Figure 5.10 the laminar and the turbulent regimes can be distinguished by their different slope and dependencies on the geometry and temperature gradient, respectively.

Based on the above reasoning and Figure 5.10 the following assumptions are made in the model:

- Channel 1 and Channel 2 are of similar length, whereas their diameter will be different. Because the cable sample in the present study has a machine-wound 
insulation it is anticipated that the winding is tighter and better controlled than in the hand-wound samples of Granieri shown in Figures 5.7 and 5.8 . On the broad face the insulation layers will be tighter and somewhat further compressed, whereas on the small face the layers will be separated because of the buckling of the insulation. As a result the diameter of channel 1 will be larger than the diameter of channel 2. In the best case, when channel 3 does not have a thermal resistance, the temperature difference $\Delta T$ of channel 1 and channel 2 are identical. This leads to the conclusion that the temperature gradient is equal in both channels or the temperature gradient in channel 1 is higher than the temperature gradient in channel 2. This information allows to draw conclusions on the heat transfer regimes of the two channels 1 and 2 . The possible combinations are that both channels are laminar, both channels are fully turbulent or that channel 1 is fully turbulent and channel 2 is in the laminar heat transfer regime. The possibility of channel 2 being turbulent and channel 1 being laminar can be excluded.

- Channel 2 and channel 3 are also of similar length, while channel 3 has a much bigger diameter than channel 2. Both channels transport the same heat flux, since the two channels are in series. As a consequence the heat flux density in channel 3 is lower than in channel 2. Since the heat flux density scales with $d^{-2}$, it can be seen from Figure 5.5 that if at a specific heat flux density channel 2 is in the laminar heat transfer regime then also channel 3 is in the laminar heat transfer regime.

\section{Model equations}

The temperature difference between the cable and the He bath is identical to the temperature difference along channel 1 . Since the channels 2 and 3 are connected in series they together have the same temperature difference as channel 1.

$$
\Delta T=\Delta T_{1}=\Delta T_{2}+\Delta T_{3}
$$

Furthermore the channels 2 and 3 transport the same heat flux.

$$
\dot{Q}_{2}=\dot{Q}_{3}
$$

For the heat flux transported in channels 2 the following expression can be derived from Equation 2.30 and Equation (2.35):

$$
\dot{Q}_{2}=\kappa_{2} \Theta_{2} x \Delta T,
$$

where $\kappa$ is the heat transfer regime dependent effective thermal conductivity term, $\Theta_{2}$ is the geometry term of channel 2, and the parameter $x$ represents the ratio of $\frac{\Delta T_{2}}{\Delta T_{3}}$ and is called the temperature split. $\kappa$ depends on the heat transfer regime and is calculated with the logarithmic mean temperature of the channel: 


$$
\begin{aligned}
\kappa_{\text {laminar }} & =\frac{(\rho s)^{2} T}{\beta \mu_{n}} \\
\kappa_{\text {turbulent }} & =\left(\frac{f^{-1}}{\Delta T^{2.4}}\right)^{\frac{1}{3.4}} .
\end{aligned}
$$

The geometry coefficient can also take two different forms depending on the heat transfer regime:

$$
\begin{aligned}
\Theta_{\text {laminar }} & =\frac{A d^{2}}{l}, \\
\Theta_{\text {turbulent }} & =\frac{A}{l^{\frac{1}{3.4}}} .
\end{aligned}
$$

The heat flux in channel 3 has the form

$$
\dot{Q}_{3}=\kappa_{3} \Theta_{3}(1-x) \Delta T \text {. }
$$

Since the channels 2 and 3 transport the same heat flux it is now possible to find an expression for the temperature split $x$ :

$$
x=\frac{\kappa_{3} \Theta_{3}}{\kappa_{3} \Theta_{3}+\kappa_{2} \Theta_{2}},
$$

which is weakly dependent on $\Delta T$ due to the temperature dependency of the effective thermal conductivity of the two channels. Since the temperature used to calculate the effective thermal conductivity is again dependent on the temperature distribution along the channel, Equation (5.19) is an implicit definition, and $x$ needs to be calculated iteratively.

The heat flux in the channel 1 is calculated as:

$$
\dot{Q}_{1}=\kappa_{1} \Theta_{1} \Delta T
$$

\subsubsection{Model Summary}

Figure 5.11 shows an illustration of the model with all the different heat transfer paths that contribute significantly to the cooling of the cable.

Two parallel helium channels represent the different channel combinations that exist in the polyimide wrapping. Solid conduction is considered only on the small face combined with the Kapitza resistance on both sides of the insulation. The contribution of solid conduction through the broad face is neglected based on Equation 5.10 on Page 61.

The effective thermal conductivities of the different helium channels are calculated with the direct transition model using the presented equations for the different channels. The thermal conductivity of the polyimide insulation and the Kapitza resistances at the boundaries are taken into account but do not include any fitting 


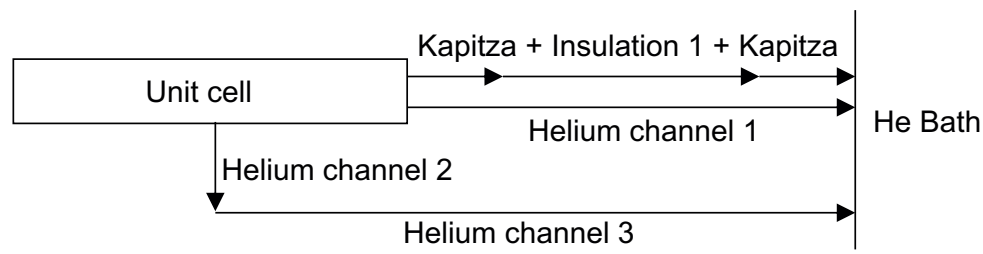

Figure 5.11. Illustration of the model with the different heat transfer paths considered in the model. The arrows are not to scale and only indicate the different paths between the heated unit cell and the constant temperature He bath.

parameter, resulting in an analytical expression for the contribution of the insulation. The geometry of the helium channels on the other hand can only be estimated with difficulties and it was decided to make no assumptions on any of the dimensions but instead determine their value with a fitting routine.

\subsubsection{Fitting Process}

Aside from the geometric parameters of the helium channels (i.e. $\Theta_{1}, \Theta_{2}, \Theta_{3}$ ) no other free parameters are used in the fitting process. All the different dimensions of the three channels appear as products of each other and it is thus impossible to fit each of the parameters individually. Instead only one global variable remains for each channel characterising the geometry.

During the fitting process all data pairs of applied heating power and temperature rise for one He bath temperature and a single mechanical pressure are fitted at once. This causes the model to be overdetermined since at least six data points are fitted with only three free variables.

The solving process of the model is iterative. First the heat flux transported by the insulation at the small face (solid conduction + Kapitza resistance) is calculated and subtracted from the total deposited heat flux. This is done before the fitting process is started and determines the heat flux that is transported by the helium channels. As a first step in the fitting process appropriate initial conditions are chosen to calculate the effective thermal conductivity of each channel. As an initial temperature split between the channels 2 and 3 a split of $50 \%$ is used. Initially, both helium thermal paths (channel 1 and channel $2+3$ ) are assumed to have the same total helium heat flux. This total helium heat flux is only used in the first iteration of the process and is replaced by the residual heat fluxes in subsequent iterations. Figure 5.12 explains the fitting process schematically from the initialisation to the final fitting coefficients.

When the fit has converged the geometric parameters are extracted. The final result of the fit process takes the form:

$$
\overrightarrow{\dot{Q}}_{\text {tot }}=\overrightarrow{\dot{Q}}_{\text {polyimide }}+\vec{\kappa}_{1} \Theta_{1} \Delta \vec{T}+\left(\left(\vec{\kappa}_{2} \Theta_{2}\right)^{-1}+\left(\vec{\kappa}_{3} \Theta_{3}\right)^{-1}\right)^{-1} \overrightarrow{\Delta T}
$$




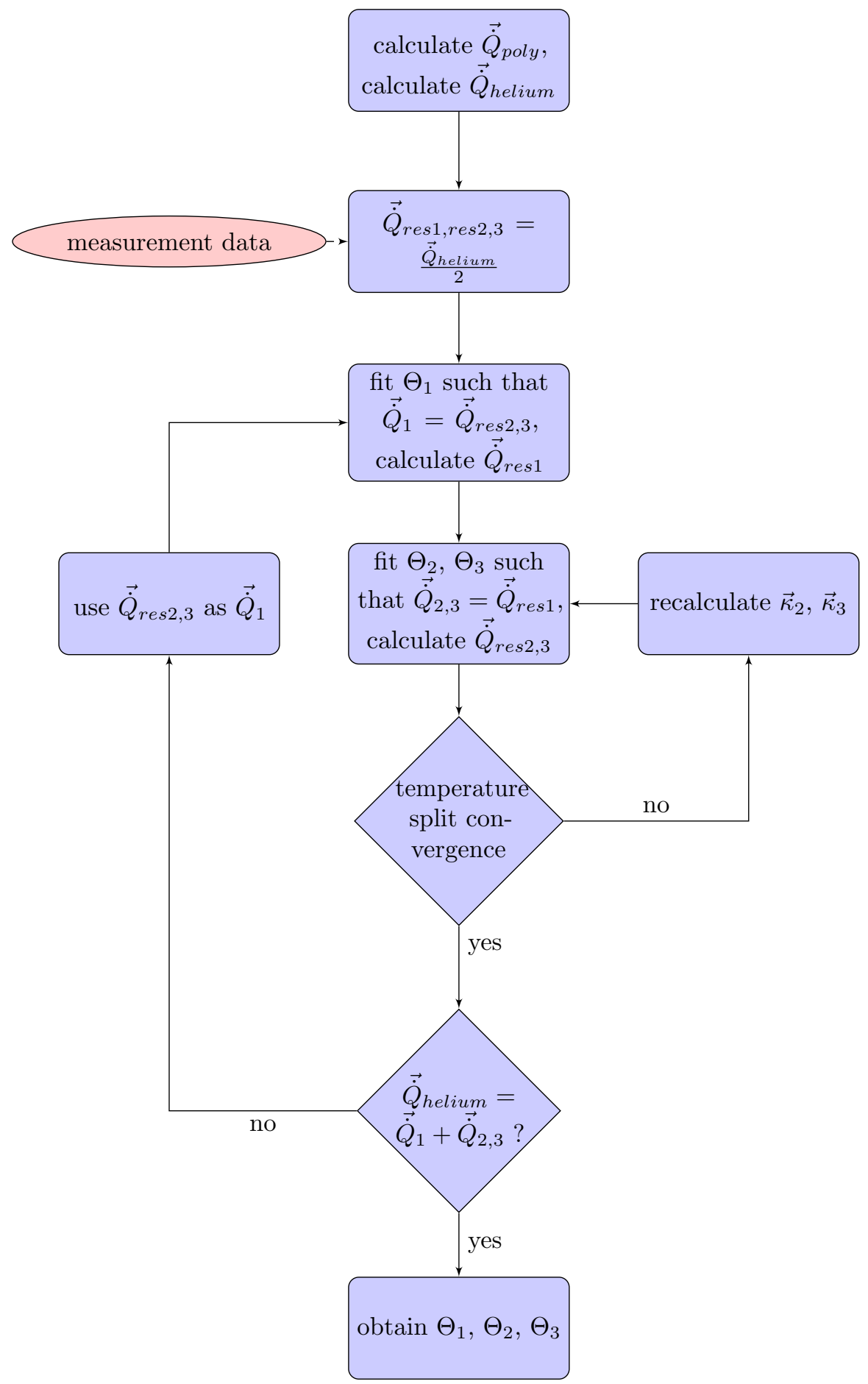

Figure 5.12. Flow chart of the fitting process. 
where $\Theta_{1}, \Theta_{2}, \Theta_{3}$ are the free geometry parameters of the three channels that are determined by the fitting routine.

The thermal properties of helium used in the fitting process are taken from the HePakC 73 database with the exception of the $f^{-1}$ function, which is taken from Sato et al. [41. The temperature at which the helium properties are calculated is the logarithmic mean temperature of each channel. 



\section{Results}

The results obtained from the measurement campaign and the comparison with the model are presented in this chapter. Each individual measurement curve is analysed as described in Section 3.4 and two fit parameters are obtained for each individual measurement. One fit parameter is the steady-state temperature difference between the superconducting cable sample and He bath, the second parameter is the characteristic time, describing the transient process of the experiment.

First the results for steady-state conditions are discussed and related to the mechanical compression, the He bath conditions and the He bath temperature. In the second part the results of the transient measurements are discussed in detail. The discussion uses the data set obtained for a mechanical sample pressure of $50 \mathrm{MPa}$ and for saturated He bath conditions unless explicitly stated otherwise. Other conditions were measured as well. The steady-state temperature differences and the time constants of the measurements for other conditions can be found in the appendix pages 103 to 106 .

\subsection{Steady State Measurement Results}

\subsubsection{Measurement Data Analysis}

Figure 6.1 shows the steady-state temperature difference between the sample and the He bath as a function of the He bath temperature at different heat loads. This exemplary graph shows the dataset obtained in saturated He II at a mechanical sample pressure of $50 \mathrm{MPa}$. The steady-state temperature difference is increasing with an increasing heating power for a given He bath temperature. The absolute temperature increase is a function of the He bath temperature for a given heating power. The temperature rise is the highest in the measured temperature range at a He bath temperature of $1.7 \mathrm{~K}$. The He bath temperature for which the lowest temperature increase is measured on the contrary is a function of the applied heating power. The minimum for the lowest applied heating power is at a He bath temperature of $1.95 \mathrm{~K}$ and shifts for an increasing heating power to higher He bath temperatures. At the highest heating power of $4.97 \mathrm{~mW} \mathrm{~cm}^{-3}$ the minimum is at a He bath temperature of $2.05 \mathrm{~K}$. The reason for this shift is explored later.

Figure 6.2 shows the measured steady-state temperature difference between sample and He bath as a function of the applied heating power for different $\mathrm{He}$ bath temperatures at a mechanical sample pressure of $50 \mathrm{MPa}$, the same data set as shown in Figure 6.1. When connecting the respective data points in a curve, then the slope of the temperature difference curve is small for an applied heating power lower than $1.1 \mathrm{~mW} \mathrm{~cm}^{-3}$ and it increases until it reaches a linear behaviour for a heating power above $2.5 \mathrm{~mW} \mathrm{~cm}^{-3}$. 


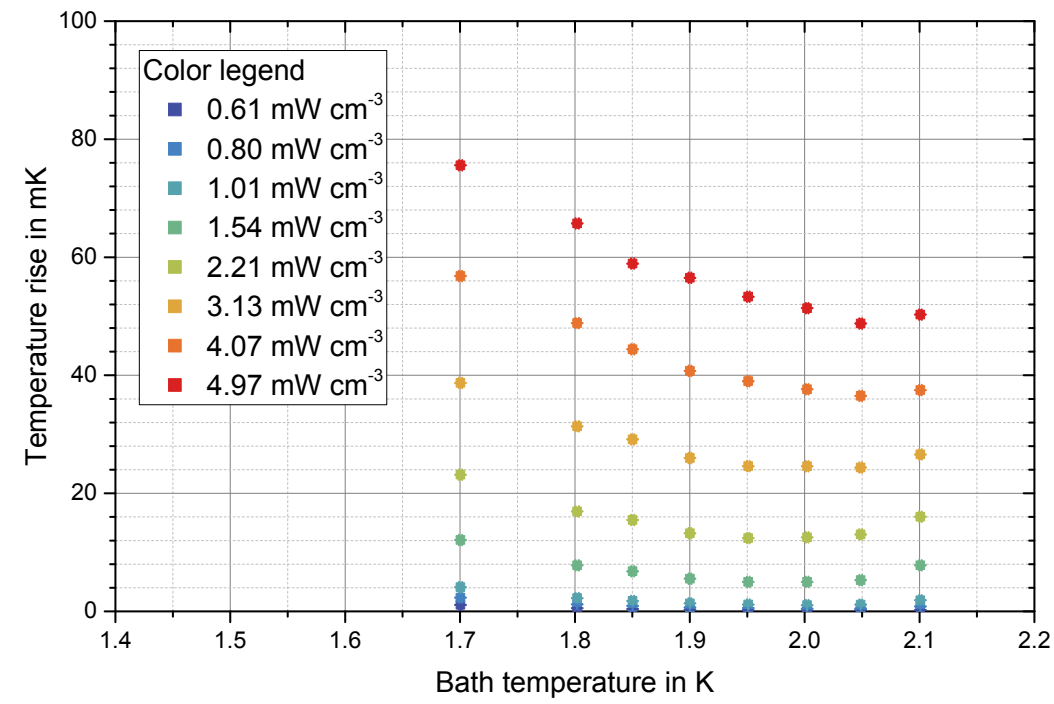

Figure 6.1. The steady-state temperature difference between the sample and the bath versus the He bath temperature at different heating loads. The displayed data set is measured in saturated He II at a mechanical sample pressure of $50 \mathrm{MPa}$ on the sample stack. The error of the applied heating power ranges from $0.16 \mathrm{~mW} \mathrm{~cm}^{-3}$ at the lowest heating power to $0.18 \mathrm{~mW} \mathrm{~cm}^{-3}$ at the highest heating power.

The turbulent heat transfer regime of He II has a strongly non-linear behaviour (see Equation (2.35)):

$$
\dot{q}^{3.4} \propto \Delta T,
$$

where $\dot{q}$ is the heating power and $\Delta T$ the steady-state temperature difference between the sample and the He bath. Figure 6.3 shows the same data set as Figure 6.2 but scaled according to Equation (6.1). For the three lowest heating powers, at a given He bath temperature, the curve of the temperature difference is linear and is passing through the origin indicating that in this range the turbulent heat transfer regime is dominant. With some extrapolation the linear behaviour is visible up to a heating power of $1.5 \mathrm{~mW} \mathrm{~cm}^{-3}$. For high heating power the slope decreases and shows again linear behaviour after the transition but an extrapolation of this linear trend line does not pass through the origin of the graph.

Considering Equation (3.14), which is obtained from the first order model for the superconducting cable cooling, the steady-state temperature difference is used together with the applied heating power to calculate the effective thermal conductivity, which is inverse of the effective thermal resistance $R$ used in the model description. Figure 6.4 shows the effective thermal conductivity as a function of the He bath temperature. For a given He bath temperature the effective thermal 


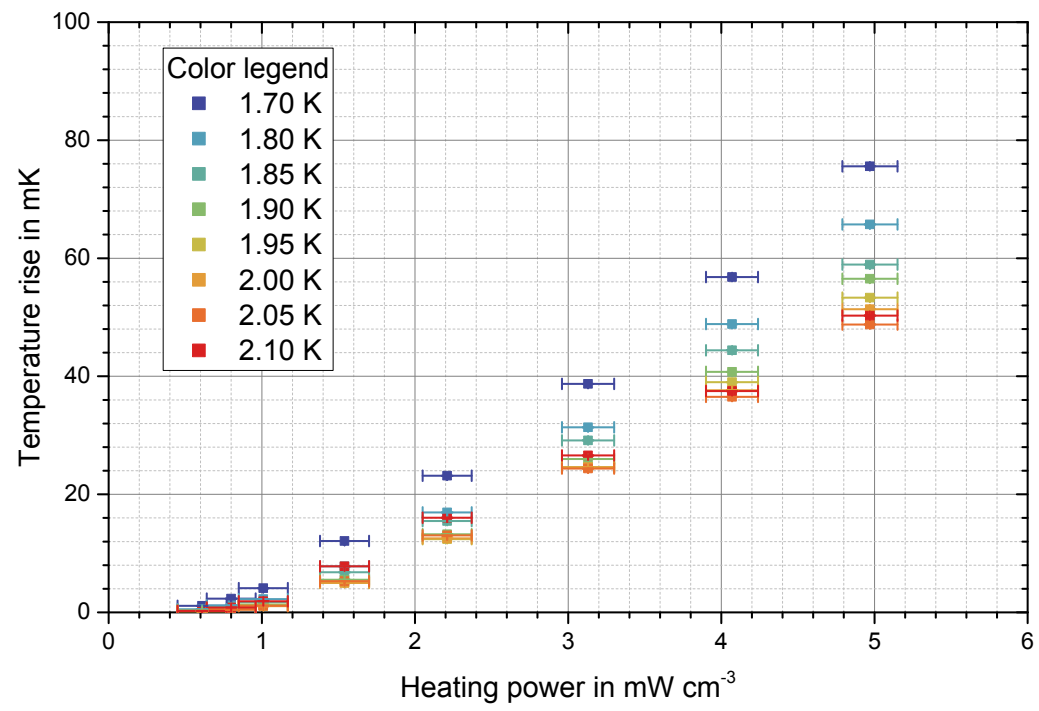

Figure 6.2. The steady-state temperature rise in the sample versus the applied heating power. Different colours denote different He bath temperatures. The displayed data set is identical to the data set in Figure 6.1

conductivity is highest for the lowest heating power and decreases with increasing heating power.

For a given heating power the order of the points confirms the previous conclusion that the four lowest heating powers are dominated by the turbulent heat transfer regime. The characteristic shape of the $f^{-1}$ function (see Figure 2.6) is clearly visible with the peak occurring at a He bath temperature of about $2.0 \mathrm{~K}$ and decreasing effective thermal conductivity values for higher and lower He bath temperatures.

With an increasing heating power the effective thermal conductivity is decreasing and the curves for a given heating power show a smaller peak until at a heating power of $4.97 \mathrm{~mW} \mathrm{~cm}^{-3}$ it not visible anymore in the data. For $4.97 \mathrm{~mW} \mathrm{~cm}^{-3}$ the effective thermal conductivity is instead monotonously increasing with an increasing He bath temperature. This monotonously increasing effective thermal conductivity suggests that a second heat transfer path contributes significantly to the cable cooling and that this second heat transfer path is in the laminar heat transfer regime of He II.

As discussed in Section 2.3.2 the turbulent heat transfer can be expected to peak at $1.9 \mathrm{~K}$. In this experiment a slightly higher peaking temperature is found of about $2.0 \mathrm{~K}$. This difference can be explained with the second heat transfer path. The second heat transfer channel is not dominant at low heating power but since its effective thermal conductivity increases with temperature (see Equation (5.14)) it contributes enough to shift the peak of the highest effective thermal conductivity 


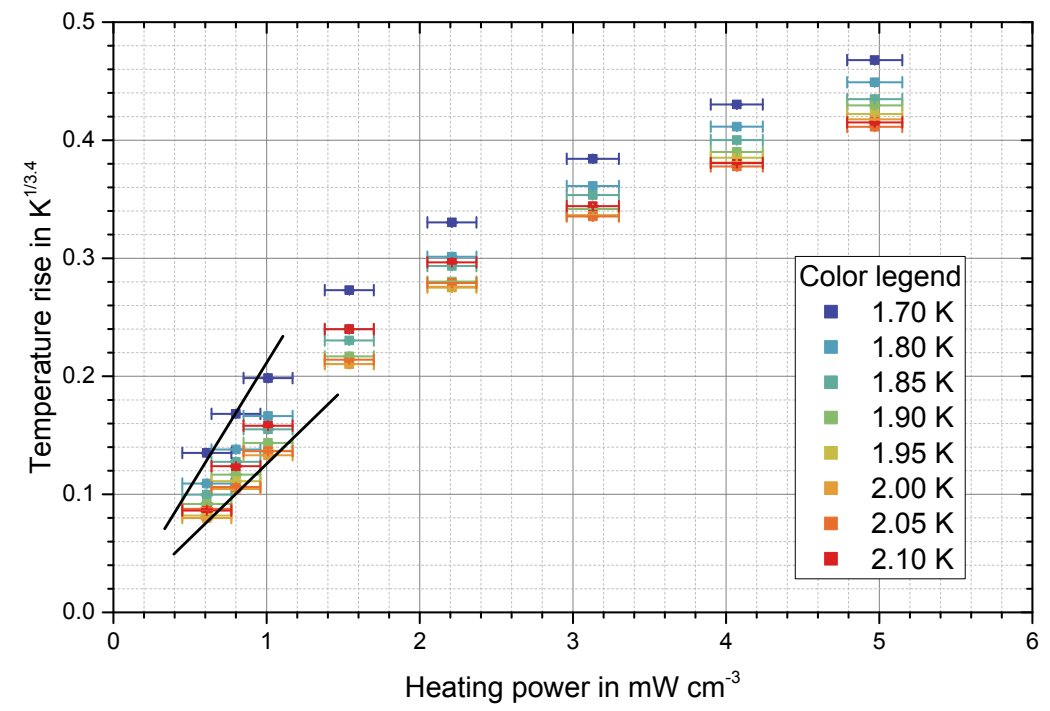

Figure 6.3. The steady-state temperature difference scaled according to Equation (6.1) versus the applied heating power. For the three lowest heating powers the linear behaviour of the temperature rise is clearly visible, indicating the contribution of turbulent He II in this heating power range. The black lines are a guide to the eye to indicate the linear proportionality.

to higher temperatures.

An explanation for the decreasing effective thermal conductivity with an increasing applied heating power is found in the dependencies of the effective turbulent thermal conductivity. The dependencies in the two heat transfer regimes in He II are the following (see Equations 2.30) and (2.35):

$$
\begin{aligned}
k_{\text {eff,laminar }} & =\frac{d^{2}(\rho s)^{2} T}{\beta \mu_{n}}=\mathrm{f}(T, d), \\
k_{\text {eff,turbulent }} & =\left(\frac{f(T)^{-1}}{\left(\frac{\Delta T}{l}\right)^{2.4}}\right)^{\frac{1}{3.4}}=\mathrm{f}\left(T, \frac{1}{\frac{\mathrm{d} T}{\mathrm{~d} x}}\right) .
\end{aligned}
$$

The two effective thermal conductivities are discussed in detail in Section 2.3.2, A higher temperature gradient is the result of an increasing heating power, which causes the effective thermal conductivity in the turbulent regime to decrease. At a certain point this effective thermal conductivity has reached such a low value that the second heat transfer path becomes the dominant contributor to the cable cooling.

Taking the different proportionalities of the two heat transfer regimes into 


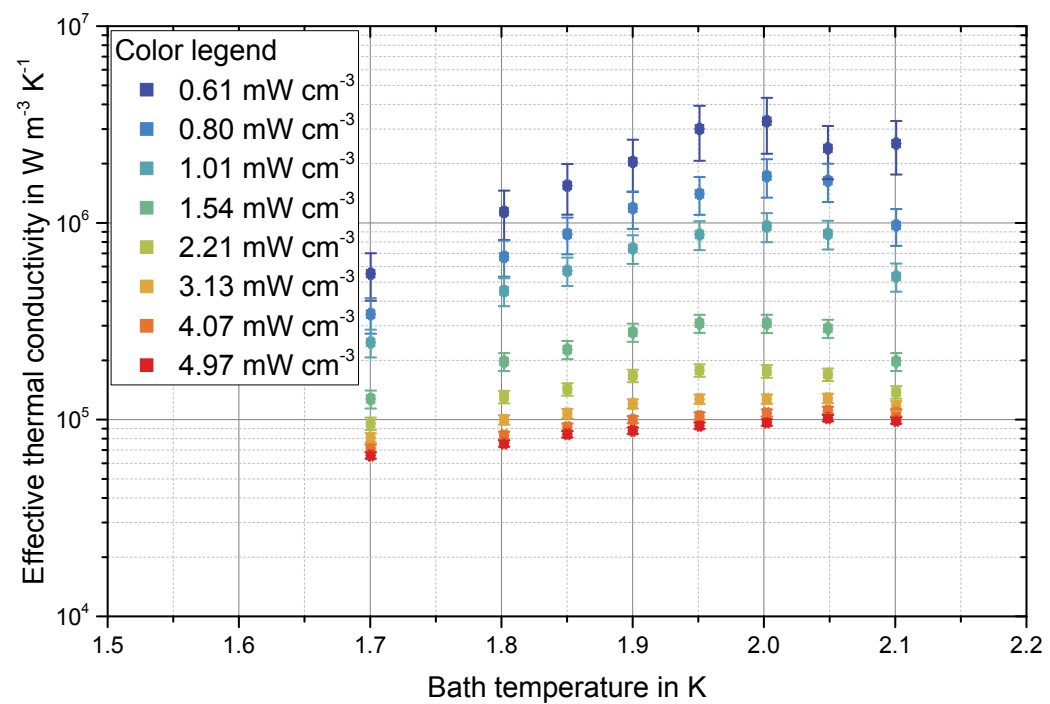

Figure 6.4. The effective thermal conductivity versus the He bath temperature. The unit for the effective thermal conductivity is a result of the heating power unit and the unknown surface area that contributes to the cooling of the unit cell of a superconducting Rutherford cable.

account, conclusions can be drawn on the composition of the heat transfer involved in the cooling of the sample. The appearance of the power of 3.4 proportionality at lower heating power indicates that at least one channel in the polyimide insulation exists that is filled with He II. When comparing the thermal conductivity of the polyimide insulation with the effective thermal conductivity determined in the experiment it also becomes clear that at least one other He channel must exist in order to obtain the right order of magnitude in effective thermal conductivity. It seems to be counterintuitive at the first glance, considering that in a single channel first the laminar regime occurs, which then transitions into the turbulent regime. This order of appearance indicates that at least two different heat transfer paths exist with two different geometric scales. One heat transfer path is already at low heating power in the turbulent regime, whereas a second heat transfer path is still in the laminar heat transfer regime even for the highest measured heating power. At a heating power of $\approx 1.5 \mathrm{~mW} \mathrm{~cm}^{-3}$ the contribution of the second heat transfer path becomes dominant over the first heat transfer path and thus for high heating power the temperature rise as function of heating power shows an almost linear behaviour with a slope of $\dot{q}^{1}$ in Figure 6.2 .

This basic data analysis of the steady-state temperature rise of the sample can be done for all different measurement runs. The absolute numbers will differ between different measurement runs but the basic conclusions are identical. After this basic introduction, supporting the assumptions made for the thermal model, the 


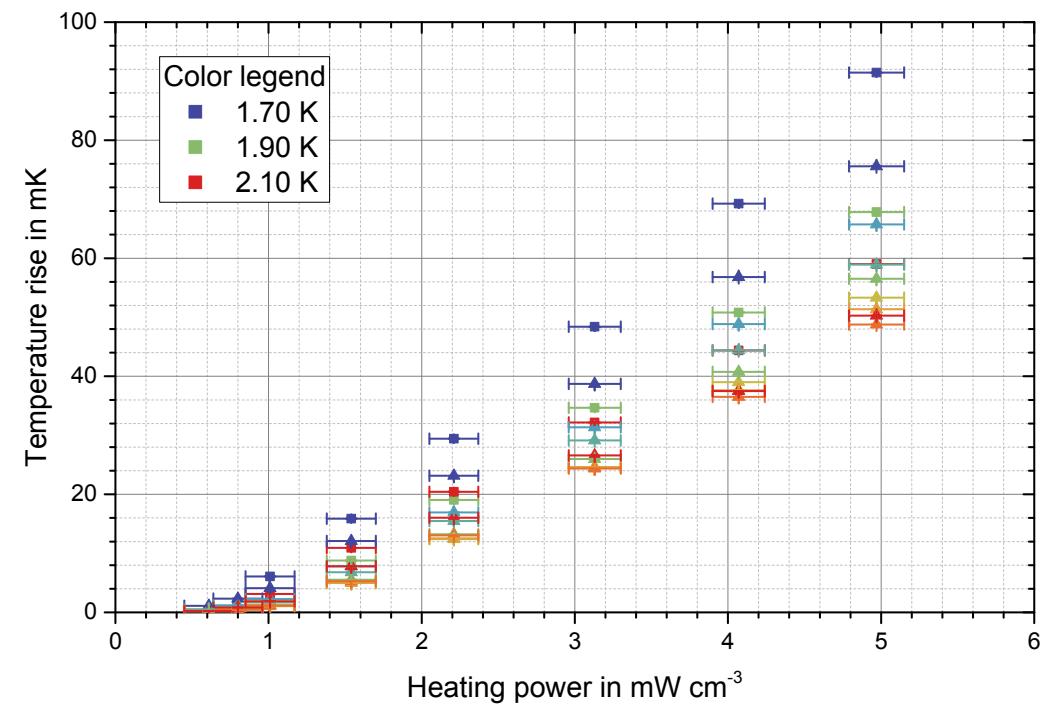

Figure 6.5. The steady-state temperature difference of the sample versus the applied heating power for a mechanical sample pressure $50 \mathrm{MPa}$. The data was obtained during two separate measurement campaigns. The figure shows the repeatability of a measurement, where the variations are due to the different thermal shrinkage and change of the friction parameter between the sample holder and the compression screws between two different measurement campaigns.

influence of different measurement conditions are discussed.

\subsubsection{Experimental Reproducibility and Accuracy}

\section{Measurement reproducibility}

To check the reproducibility of the measured steady-state temperature difference the set-up was warmed up between two measurement runs and the mechanical sample pressure of the sample was set to the same initial conditions. Then the sample was cooled down a second time and the measurements were repeated:

Figure 6.5 shows the temperature difference data as a function of heating power. The data in this figure is from two different measurement runs where the sample was warmed up to room temperature between the two runs. In both cases the sample was tightened with the same torque on the compression screws before the cool-down. Nonetheless a spread of up to $20 \%$ between measurement data for the same applied heating power is visible. The reason for this difference between two measurement runs is attributed to a different thermal shrinkage between measurement runs, which is a result of small movements of the sample during the cool-down and warm-up, and due to the uncertainty of the tightening process 


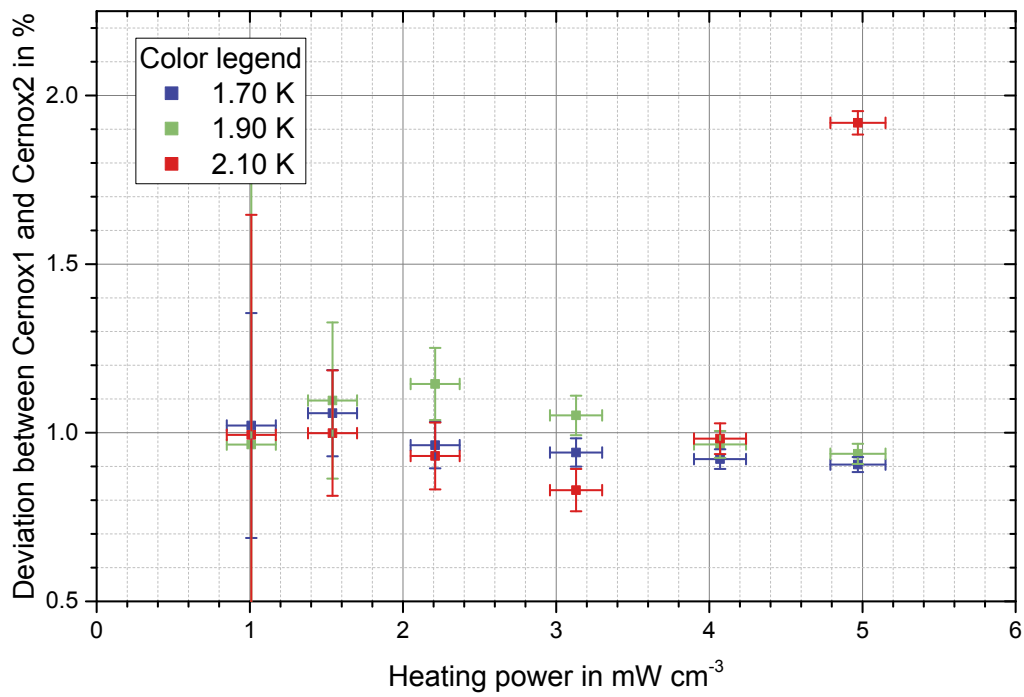

Figure 6.6. The deviation between the two Cernox ${ }^{T M}$ temperature sensors that are placed in the sample for a mechanical sample pressure of $50 \mathrm{MPa}$. With the exception of one point at $2.1 \mathrm{~K}$ at maximum applied heating power, all points show a systematic difference of $\approx 1 \%$ between the temperatures measured at the point of maximum mechanical sample pressure in the sample and the point of the lowest mechanical sample pressure along the centre line of the superconducting sample stack. The outlier in this figure is related to the temperature of the sample getting close to the $\lambda$ transition.

where a constant friction coefficient between the screws and the sample holder is assumed. The friction coefficient between the screw and the sample holder is not constant but changes with the continued use of the sample holder.

\section{Mechanical compression homogeneity}

Since the compression screws are mounted with some space between each other along the sample length a variation in the compression force on the sample could occur. To quantify the variation of the compression on the measured temperature difference the two sensors were placed in two different locations. The first sensor, Cernox1, was placed directly under a compression screw, where the mechanical sample pressure is the highest. The second sensor, Cernox2, was placed between two compression screws, where the mechanical sample pressure is lowest.

Figure 6.6 shows the measurement data for the two sensors for a mechanical compression of $50 \mathrm{MPa}$. The absolute difference between the two sensors is smaller than $1 \mathrm{mK}$. The systematic deviation of $\approx 1 \%$ between the two sensors is independent of the measurement conditions, except the $2.1 \mathrm{~K}$ point at full power. It can 


\section{Results}

be seen that the pressure homogeneity in the sample compression shows variations that influence the steady-state sample temperature by roughly $1 \%$. A variation much smaller than the total temperature increase and as a conclusion the variations in the homogeneity can thus be neglected.

\section{Measurement reproducibility conclusion}

It can be concluded that the measurement results show a variation of at least $20 \%$ between different measurement runs. This is caused by a slightly varying mechanical compression between two different measurement runs and the uncertainty related to the tightening process of the sample. Nonetheless, the qualitative behaviour of all measurement runs is independent of the reproducibility between two different measurement runs.

It can also be concluded that the sample holder provides a homogeneous compression of the sample within one measurement run. The measurement of one temperature in the sample is therefore considered to be sufficient.

\subsubsection{Model Data Analysis}

The heat transfer for each experiment is modelled with the model developed in Chapter 5. To demonstrate the capabilities of the model the measurement result obtained for a He bath temperature of $1.9 \mathrm{~K}$ in saturated He II is discussed in this section. Firstly the heat flux composition is discussed and then the heat flux split between channel 1 and channel $2+3$.

\section{Heat flux composition}

Figure 6.7 shows an illustrative graph for the model at $1.9 \mathrm{~K}$ for a mechanical sample pressure of $50 \mathrm{MPa}$. It shows the contribution of the different heat flow channels to the heat flux together with the sum of the different channels. The polyimide contribution is increasing with increasing heating power and stays in the order of $10 \%$ in the investigated heating power and temperature ranges. Channel 1 , which is located on the small face of the cable, shows a strongly non-linear behaviour and based on previously mentioned reasons, it is expected to be in the turbulent He II heat transfer regime. The majority of the heat flux at lower heating power is transported by this channel. For an increasing heating power the thermal resistance of this channel is increasing due to the increasing temperature gradient, which reduces the effective thermal conductivity in the turbulent heat transfer regime, and channel 2, which shows an almost linear behaviour, is becoming the dominant heat transfer path. The obtained linear proportionality between the temperature rise and the applied heating power is due to fact that channel 2 is in the laminar heat transfer regime.

Table 6.1 lists the geometry parameters that were obtained from the fit of the model to the measurement data at $1.9 \mathrm{~K}$ in a saturated He bath for a mechanical sample pressure of $50 \mathrm{MPa}$. 


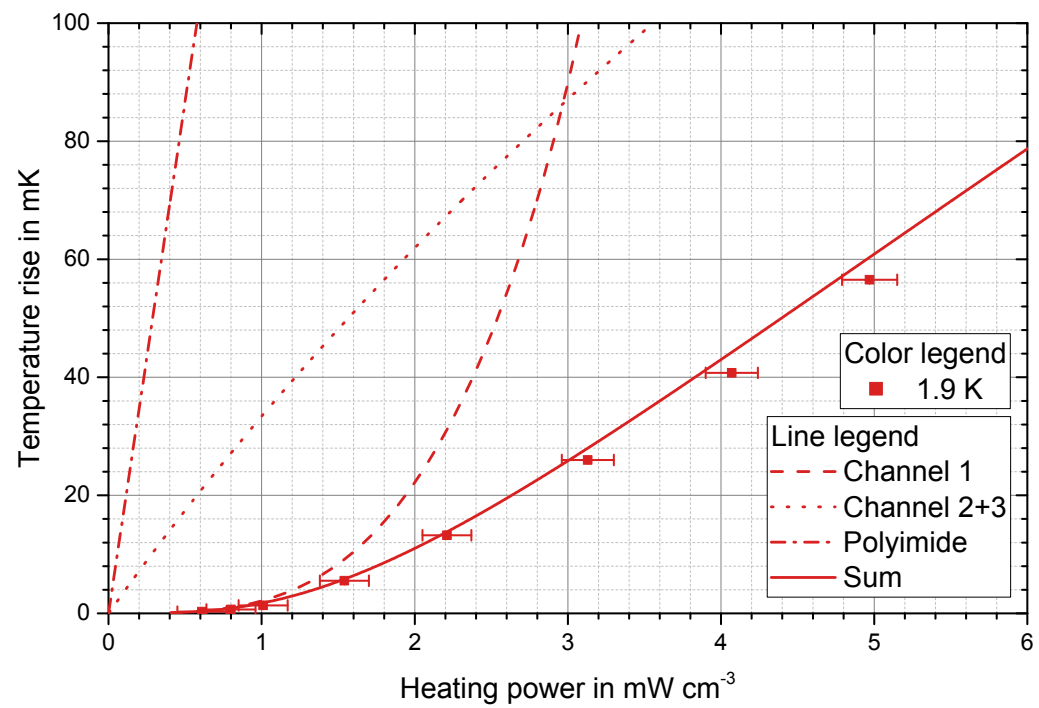

Figure 6.7. Comparison of the results of the $50 \mathrm{MPa}$ measurement run with the modelled heat flux that is transported by each channel individually. The sum of the heat fluxes is shown together with the experimental data used for the fitting process. The solid conduction through the polyimide insulation has to smallest contribution to the total heat flux. Channel 1 shows the characteristic behaviour for the turbulent heat transfer regime. The behaviour of channel 2 is characteristic for the laminar heat transfer regime.

\section{Heat flux split}

The two parallel channels 1 and $2+3$ together with the different heat transfer regimes and dimensions lead to a heat flux split between the two channel combinations that depends on the temperature difference. Figure 6.8 shows the percentage of the total heat flux that is transported by the two He channels, channel 1 and channel $2+3$. The clearly visible effect of the temperature rise on the heat flux split is explained by the fact that channel 1 is in the turbulent regime and channel $2+3$ in the laminar regime. Channel 1 is very sensitive to the temperature gradient. The effect is very pronounced at $2.1 \mathrm{~K}$ where the effective thermal conductivity is dropping steeply in the turbulent regime, whereas the effective thermal conductivity in the laminar regime is still increasing, see Section 2.3.2. Figure 6.8 also shows the contribution of the channels $2+3$ to the total heat flux as a function of the temperature difference between the superconducting cable stack and the He bath. For a He bath temperature of $2.1 \mathrm{~K}$ the contribution of channel $2+3$ is rapidly increasing up to $80 \%$ of the total heat flux, showing the increasing importance of the contribution of channel $2+3$ to the heat transfer as the He bath temperature increases as well as for an increasing applied heating power. 
Table 6.1. Fit parameters for a He bath temperature of $1.9 \mathrm{~K}$ and a mechanical sample pressure of $50 \mathrm{MPa}$ in saturated He.

\begin{tabular}{l|ccc}
$1.9 \mathrm{~K}$ & $\Theta_{1}=\frac{A_{1}}{\frac{1}{l^{3.4}}}$ & $\Theta_{2}=\frac{A_{2} d_{2}^{2}}{l_{2}}$ & $\Theta_{3}=\frac{A_{3} d_{3}^{2}}{l_{3}}$ \\
$50 \mathrm{MPa}$ & in $\mathrm{m}^{\frac{5.8}{3.4}}$ & in $^{3}$ & in $^{3}$ \\
\hline \hline sat He II & $1.61 \times 10^{-8}$ & $3.30 \times 10^{-18}$ & $7.30 \times 10^{-15}$
\end{tabular}

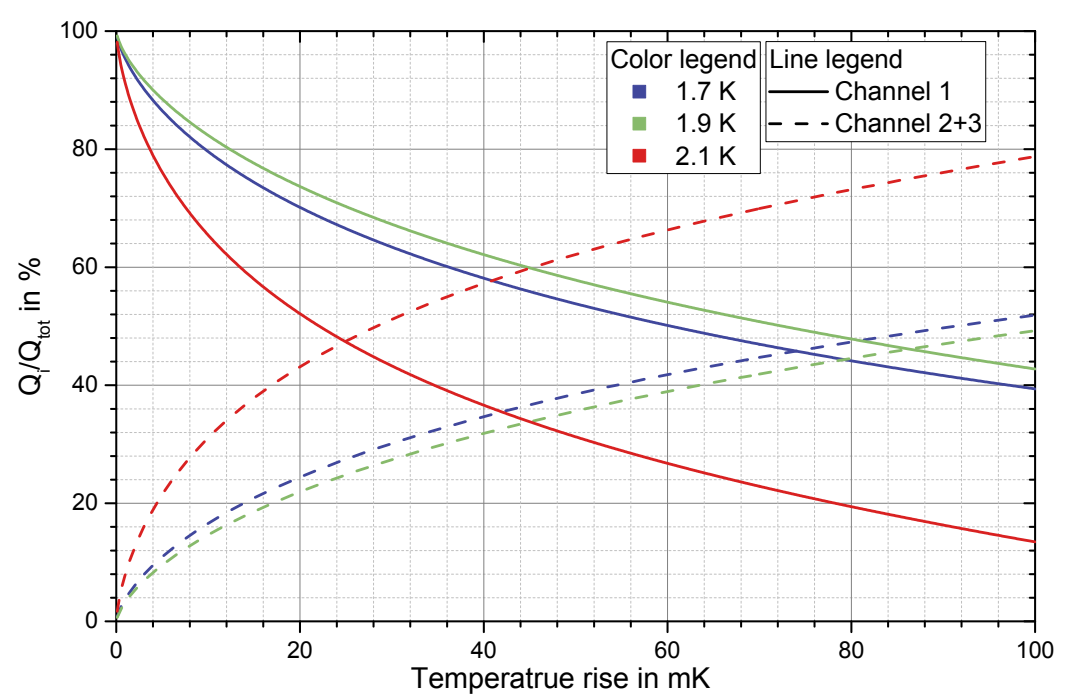

Figure 6.8. The calculated percentage of total heat flux transported by channel 1 and channel $2+3$ as a function of temperature rise for three $\mathrm{He}$ bath temperatures $1.7 \mathrm{~K}, 1.9 \mathrm{~K}$ and $2.1 \mathrm{~K}$ at a mechanical sample pressure of $50 \mathrm{MPa}$. The missing contribution to $100 \%$ is the polyimide contribution.

\subsubsection{Different Experimental Conditions}

To study the heat transfer as a function of different environmental conditions the He bath was changed between a saturation conditions and sub-cooled conditions. In a second study the experiment was conducted for two mechanical sample pressures. the results of these experiments are shown in the following paragraphs.

\section{Saturated He II compared to pressurised He II}

Figure 6.9 shows the measurement results for saturated and pressurised He II for a mechanical sample pressure of $50 \mathrm{MPa}$. The two different sets of measurement were 


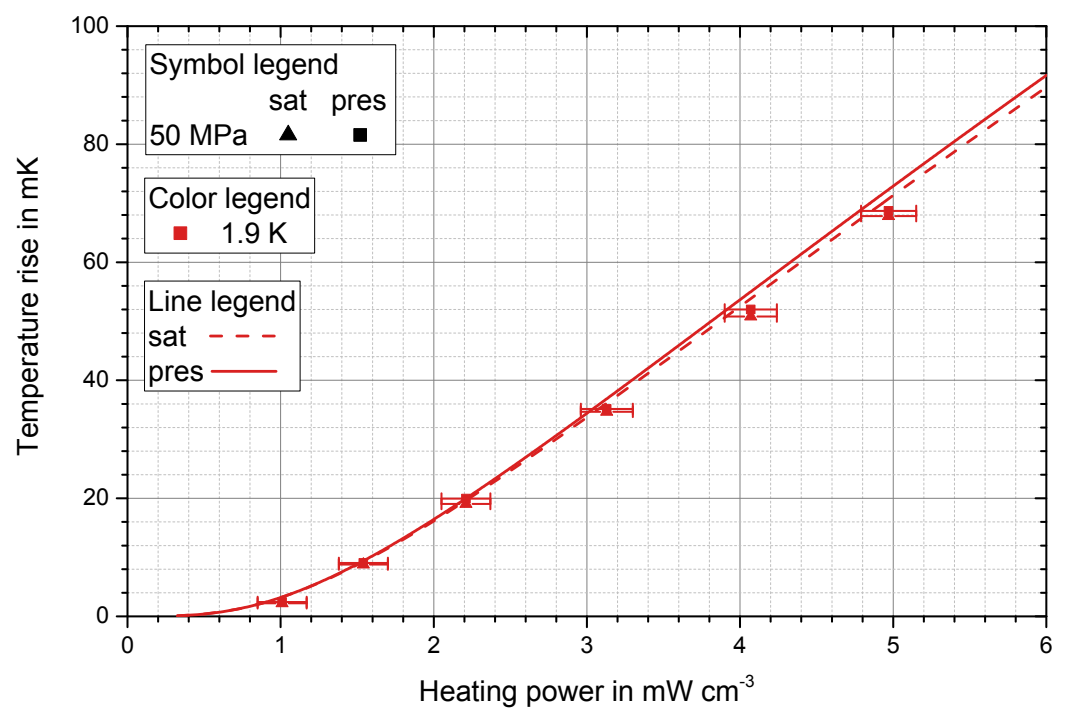

Figure 6.9. The model and the measurement data for a mechanical sample pressure of $50 \mathrm{MPa}$ for saturated He II and pressurised He II. The points denote the experimentally obtained data points, the lines are the results of the model. The full line is showing the pressurised conditions and the dashed line shows the result of the modelling for saturated He II.

Table 6.2. Fit parameters for a He bath temperature of $1.9 \mathrm{~K}$ and a mechanical sample pressure of $50 \mathrm{MPa}$ for saturated and pressurised He II conditions.

\begin{tabular}{l|ccc}
$1.9 \mathrm{~K}$ & $\Theta_{1}=\frac{A_{1}}{l^{\frac{1}{3.4}}}$ & $\Theta_{2}=\frac{A_{2} d_{2}^{2}}{l_{2}}$ & $\Theta_{3}=\frac{A_{3} d_{3}^{2}}{l_{3}}$ \\
$50 \mathrm{MPa}$ & in $\mathrm{m}^{\frac{5.8}{3.4}}$ & in $^{3}$ & in $^{3}$ \\
\hline \hline & $1.28 \times 10^{-8}$ & $3.28 \times 10^{-18}$ & $6.93 \times 10^{-15}$
\end{tabular}

obtained during the same measurement campaign and are thus not affected by the reproducibility of the mechanical compression, which is mentioned before. In this case the difference in steady-state temperature rise is solely due to the changes in the transport properties between saturated and pressurised He II. The steadystate temperature rise is increasing marginally for pressurised He II compared to saturated He II for the same experimental conditions. This indicates an increased thermal resistance for pressurised He II, which is confirmed by the values for the effective thermal conductivity of the two heat transfer regimes. The effective thermal conductivity in the turbulent regime is lower by $8 \%$ in pressurised He II at $0.12 \mathrm{MPa}$ compared to He II at saturation conditions. The effective thermal conductivity in the laminar regime for pressurised He II is decreased by about $4 \%$ 


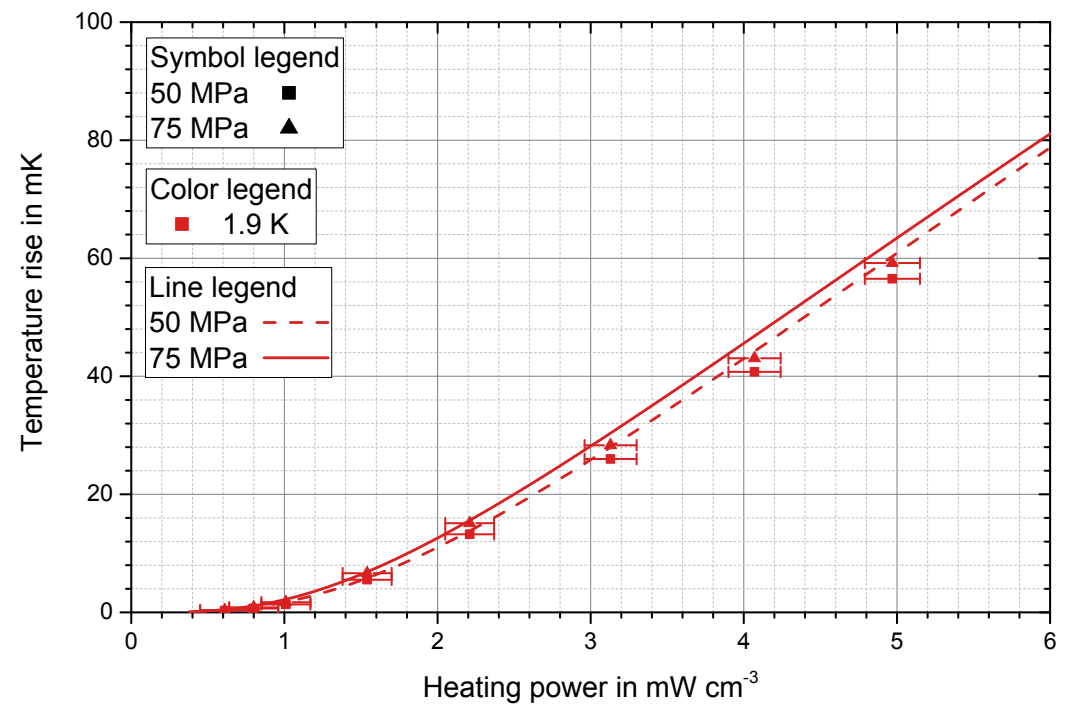

Figure 6.10. Comparison of the model and experimental data for $50 \mathrm{MPa}$ and $75 \mathrm{MPa}$ at $1.9 \mathrm{~K}$ under saturation conditions. Lines are model results and points show experimental data points.

when the He bath pressure increases from saturation pressure to $0.12 \mathrm{MPa}$. The change in the steady-state temperature difference is thus caused by the change in the transport properties of He II. Table 6.2 gives the parameters obtained from the model for saturated and pressurised He II. Because the geometry is identical independent of the He bath conditions only one set of geometry parameters was obtained.

The qualitative behaviour of the model and the measurement is reasonably good for the two experimental conditions. While taking the changed physical properties of He II into account the model does show a deviation from the measurement data especially at intermediate heating power. Considering the uncertainty with the repeatability of the measurement the model still lies within the error margin of the reproducibility.

\section{Mechanical sample pressure}

To study the effect of the mechanical sample pressure on the heat transfer between the superconducting cable stack and the He bath, two different mechanical sample pressures were applied. The results of the measurements at $50 \mathrm{MPa}$ and $75 \mathrm{MPa}$ are shown in Figure 6.10 together with the results of the model for the measurement data. Both measurement sets show similar qualitative behaviour, with the measurement data at $75 \mathrm{MPa}$ showing a marginally higher temperature increase. At a heating power of $4.97 \mathrm{~mW} \mathrm{~cm}^{-3}$ the difference in temperature rise 
is $3 \mathrm{mK}$. Comparing the predicted temperature rise curves from the model with the measured data good quantitative and qualitative agreement between the two is found. For higher applied heating power the temperature rise curves from the model predict a higher thermal resistance than the measurement data but the model prediction is nonetheless within the measurement uncertainty.

Table 6.3. Fit parameters for $1.9 \mathrm{~K}$ and for two mechanical sample pressure of $50 \mathrm{MPa}$ and $75 \mathrm{MPa}$ for saturated He II conditions.

\begin{tabular}{l|ccc}
$1.9 \mathrm{~K}$ & $\Theta_{1}=\frac{A_{1}}{l^{\frac{1}{3.4}}}$ & $\Theta_{2}=\frac{A_{2} d_{2}^{2}}{l_{2}}$ & $\Theta_{3}=\frac{A_{3} d_{3}^{2}}{l_{3}}$ \\
sat He II & in $\mathrm{m}^{\frac{5.8}{3.4}} \mathrm{in}$ & $\mathrm{m}^{3}$ & in $^{3}$ \\
\hline \hline $50 \mathrm{MPa}$ & $1.61 \times 10^{-8}$ & $3.30 \times 10^{-18}$ & $7.30 \times 10^{-16}$ \\
$75 \mathrm{MPa}$ & $1.48 \times 10^{-8}$ & $3.42 \times 10^{-18}$ & $7.85 \times 10^{-16}$
\end{tabular}

Table 6.3 gives the fit parameters obtained for two different mechanical sample pressures and the results of the fitting indicate that all channels are only affected to a small amount by the increased mechanical sample pressure. The difference between the two sets of parameters is smaller than $9 \%$ for channel 1 and even smaller for the other two channels. This confirms the finding of other research groups who found that a compression increase above $50 \mathrm{MPa}$ shows only a very small influence on the measurement results [45, 52.

When taking into account the reproducibility of the measurement as shown above this difference in the steady-state temperature difference could be related to two different effects. Either the sample geometry is changing with an increasing mechanical sample pressure or the differences is solely related to the uncertainty of the friction coefficient that translates the torque of the screws to a mechanical sample pressure on the sample. In view of these two possibilities the results are considered to be inconclusive and no statement is made on the effect of the mechanical sample pressure in the heat transfer between the superconducting cable and the cooling He bath.

\subsubsection{Temperature range $1.7 \mathrm{~K}$ to $2.1 \mathrm{~K}$}

In the previous subsection the results of the experiment and the model for different experimental conditions were presented for a He bath temperature of $1.9 \mathrm{~K}$. In this subsection the mechanical sample pressure is kept at $50 \mathrm{MPa}$ and the He bath is at saturation conditions. The He bath temperature is varied and the experimental data is compared with the model for two model cases. First the model assumes to have only one geometry parameter set for the whole temperature range. In a second study the geometry coefficients are calculated for each He bath temperature separately.

Figure 6.11 shows the model results together with the experimental data for the temperatures $1.7 \mathrm{~K}$ to $2.1 \mathrm{~K}$ in saturated He II for a mechanical sample pressure of $50 \mathrm{MPa}$ when a single set of geometry parameters is calculated. 


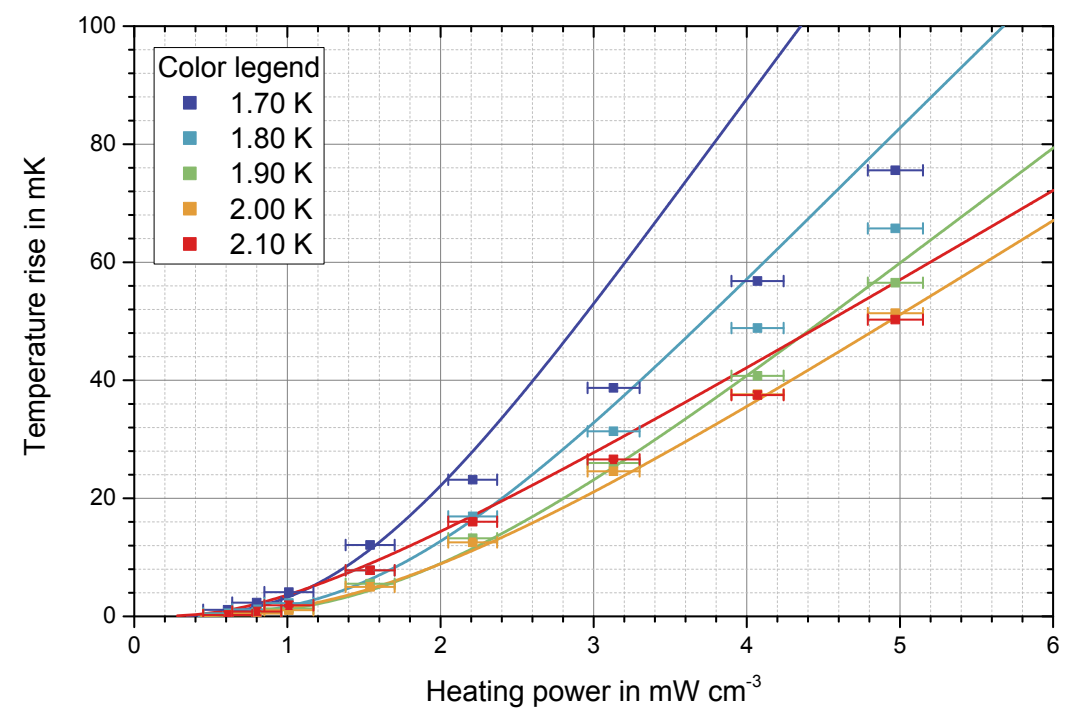

Figure 6.11. The steady-state temperature difference versus the applied heating power in the He bath temperature range $1.7 \mathrm{~K}$ to $2.1 \mathrm{~K}$ in saturated He II. Points are experimental data and lines are the model results.

As can be seen in Figure 6.11 the model gives mixed results. The fit gives good results for the temperatures around $1.9 \mathrm{~K}$ and results in deviations for He bath temperatures of $1.8 \mathrm{~K}$ and lower as well as to some small deviations for a He bath temperature of $2.1 \mathrm{~K}$.

At He bath temperatures of $1.8 \mathrm{~K}$ and lower the model predicts for small applied heating power still the characteristic slope change due to channel 1 being in the turbulent heat transfer regime. For high heating power the model predicts a too high thermal resistance resulting in too high temperature increases thus underestimating the effective thermal conductivity. The reason for this behaviour could be the heat transfer regime of channel 1 , which is calculated to be in the turbulent regime by the direct transition model but could also be in the transition regime. If channel 1 would be in the transition regime the effective thermal conductivity would be higher, which would in turn bring the model curves at bath temperatures below $1.8 \mathrm{~K}$ closer to the measurement data by the increased effective thermal conductivity.

Small deviations for higher applied heating power between the experimental data and the model predictions are found for a He bath temperature of $2.1 \mathrm{~K}$. The model fits the measurement data well for low applied heating power and predicts a linear increasing effective thermal conductivity for higher heating power. The model underestimates the effective thermal conductivity at higher heating power, which results in a higher temperature rise than the experimental data shows.

Table 6.4 lists the fit parameters from the model for the temperature range $1.7 \mathrm{~K}$ to $2.1 \mathrm{~K}$ together with previous fit parameters for the He bath temperature range $1.8 \mathrm{~K}$ to $2.0 \mathrm{~K}$ and the He bath temperature $1.9 \mathrm{~K}$ for a comparison. The difference 
Table 6.4. Fit parameters for the temperature range $1.7 \mathrm{~K}$ to $2.1 \mathrm{~K}$ and $1.9 \mathrm{~K}$ in saturated He II and a mechanical sample pressure $50 \mathrm{MPa}$

\begin{tabular}{l|ccc}
$50 \mathrm{MPa}$ & $\Theta_{1}=\frac{A_{1}}{\frac{1}{l^{3.4}}}$ & $\Theta_{2}=\frac{A_{2} d_{2}^{2}}{l_{2}}$ & $\Theta_{3}=\frac{A_{3} d_{3}^{2}}{l_{3}}$ \\
sat He II & in $\mathrm{m}^{\frac{5.8}{3.4}}$ & in $^{3}$ & in $^{3}$ \\
\hline \hline $1.7 \mathrm{~K}$ to $2.1 \mathrm{~K}$ & $1.83 \times 10^{-8}$ & $2.77 \times 10^{-18}$ & $1.01 \times 10^{-15}$ \\
$1.9 \mathrm{~K}$ & $1.61 \times 10^{-8}$ & $3.30 \times 10^{-18}$ & $7.30 \times 10^{-16}$
\end{tabular}

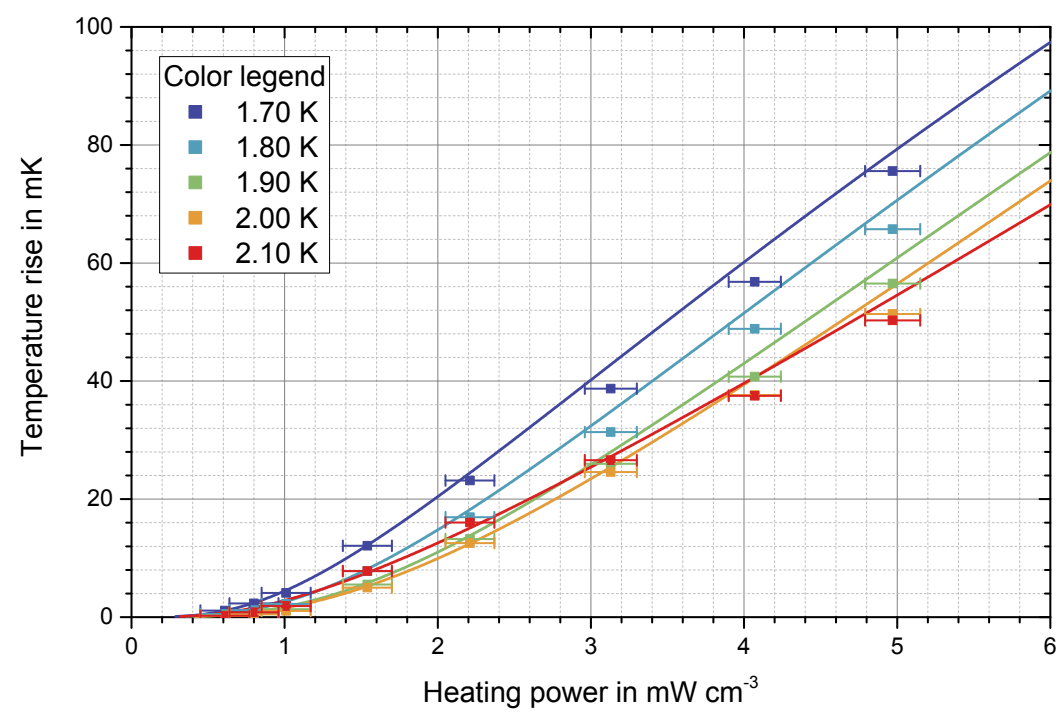

Figure 6.12. Comparison of the model with experimental data, where each He bath temperature is fitted independently. The data points are obtained with a mechanical pressure of $50 \mathrm{MPa}$ in a saturated He bath.

between the geometry parameters for the full temperature range and the fit for a He bath temperature of $1.9 \mathrm{~K}$ is small and the values are very close to each other.

\section{Fit parameter change}

Up to now only one set of fit parameters has been obtained even when increasing the temperature range. On one hand this is reasonable since the geometry itself should not show a temperature dependency in the investigated temperature range. On the other hand for the model it is assumed that different channels, which have the same location in the sample can be modelled as a single channel with an averaged geometry. This averaged geometry does not take into account small variations that could cause some of the averaged channels to show a different thermal behaviour 


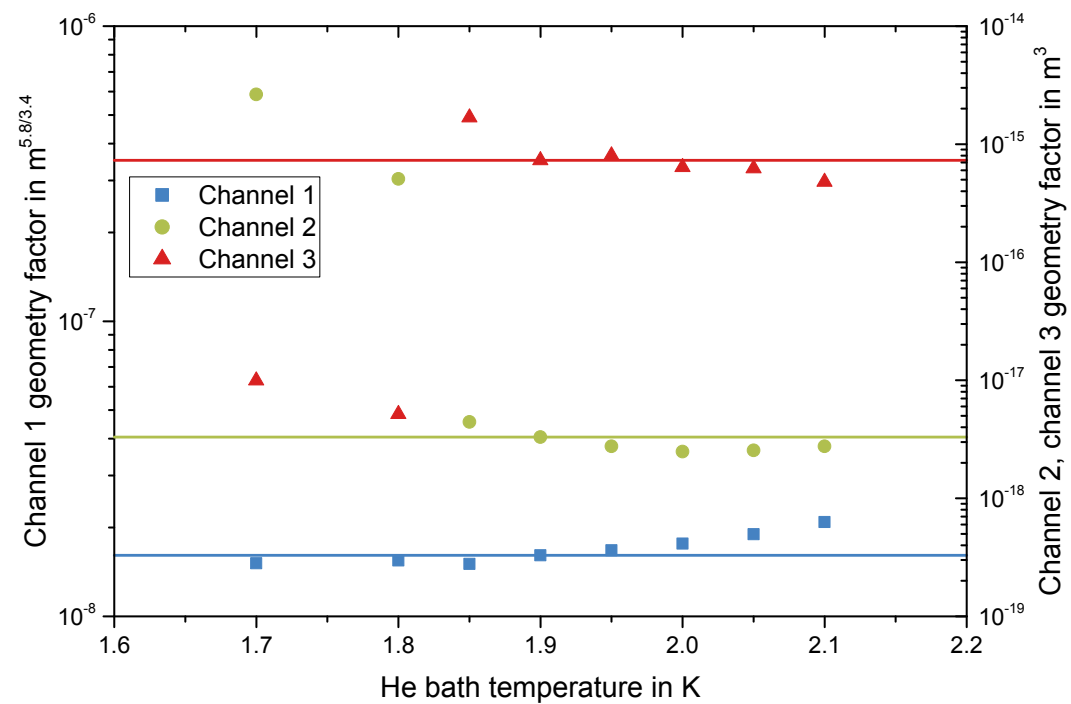

Figure 6.13. The three fit parameters representing the geometry of the different channels as a function of the He bath temperature. On the left-hand side axis the fit parameter for channel 1 is given. On the right-hand side the geometry factors of the channels 2 and 3 are given.

than the neighbouring channel. This could cause a change of the values of the model parameters for different temperatures.

To evaluate this behaviour each temperature data set for the investigated He bath temperature range is modelled individually and the results are discussed in this subsection.

Figure 6.12 shows the model results for $50 \mathrm{MPa}$ at saturation conditions. A good quantitative and qualitative agreement between the model and the data is obtained for the whole investigated temperature range of $1.7 \mathrm{~K}$ to $2.1 \mathrm{~K}$. When comparing Figure 6.12 to Figure 6.11 the difference in quality is eminent.

Figure 6.13 shows the different fit parameters of the model against the He bath temperature. The fit parameter for channel 1 has its lowest value at a temperature of $1.9 \mathrm{~K}$ and increases for higher and lower temperatures. This change of the channel 1 parameter seems to mimic the inverse shape of the $f^{-1}$-curve.

The parameters for the channels 2 and 3 are almost constant above a He bath temperature of $1.9 \mathrm{~K}$. At lower He bath temperatures the fit parameters show very high changes in their respective values. The geometric factor of channel 3 suddenly drops by almost three orders of magnitude, wheres as the geometric factor of channel 2 increases by about three orders of magnitude.

The result of this sudden change of the geometric parameter is shown in Figure 6.14. It shows the temperature split between the channels 2 and 3 as a function of the temperature difference for different He bath temperatures. The change in 


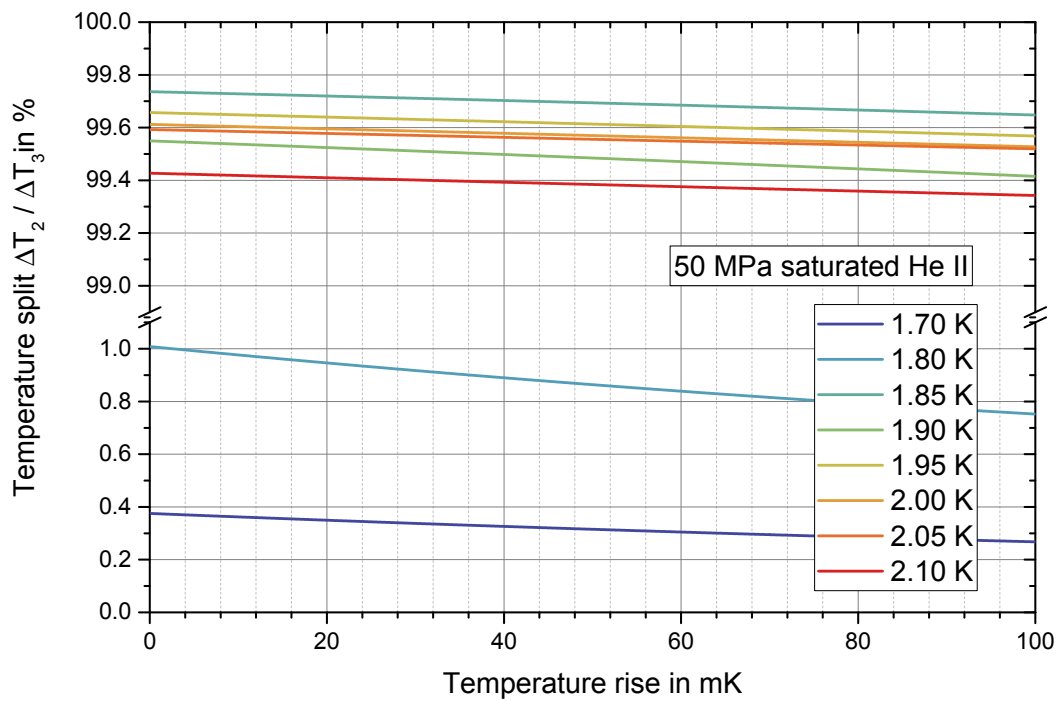

Figure 6.14. The temperature split as a function of the temperature difference for different $\mathrm{He}$ bath temperatures. As can be seen the change in the geometric parameters seen in Figure 6.13 results in a drastic change of the temperature split.

the geometric parameters of the two channels seen in Figure 6.13 results for a He bath temperature of $1.7 \mathrm{~K}$ and $1.8 \mathrm{~K}$ that most of the temperature difference occurs in channel 3 compared to the higher temperatures where most of thermal resistance is posed by channel 2 .

\section{Model refinement}

A geometry that depends to such an extent on the He bath temperature is counterintuitive and physically unreasonable. Taking a look at the split of the temperature rise between the channels 2 and 3 it also becomes eminent that at least at He bath temperatures higher than $1.9 \mathrm{~K}$ channel 3 can be neglected since it only creates less than $1 \%$ of the total temperature difference. At lower He bath temperatures it reverses and channel 2 exhibits a low thermal resistance.

Based on the changing geometry factor and the small temperature difference in either channel 2 or channel 3 it was thus decided to simplify the model further and reduce the channel combination $2+3$ to a single channel with one geometry. The measurement data were modelled with this simplified model and the result is presented in the following paragraphs and in Appendix $B$. The new fit parameters that are obtained with the simplified two channel model are given here and compared to the fit parameters obtained by the three channel model.

Figure 6.15 shows the results of the simplified two channel model when the full 


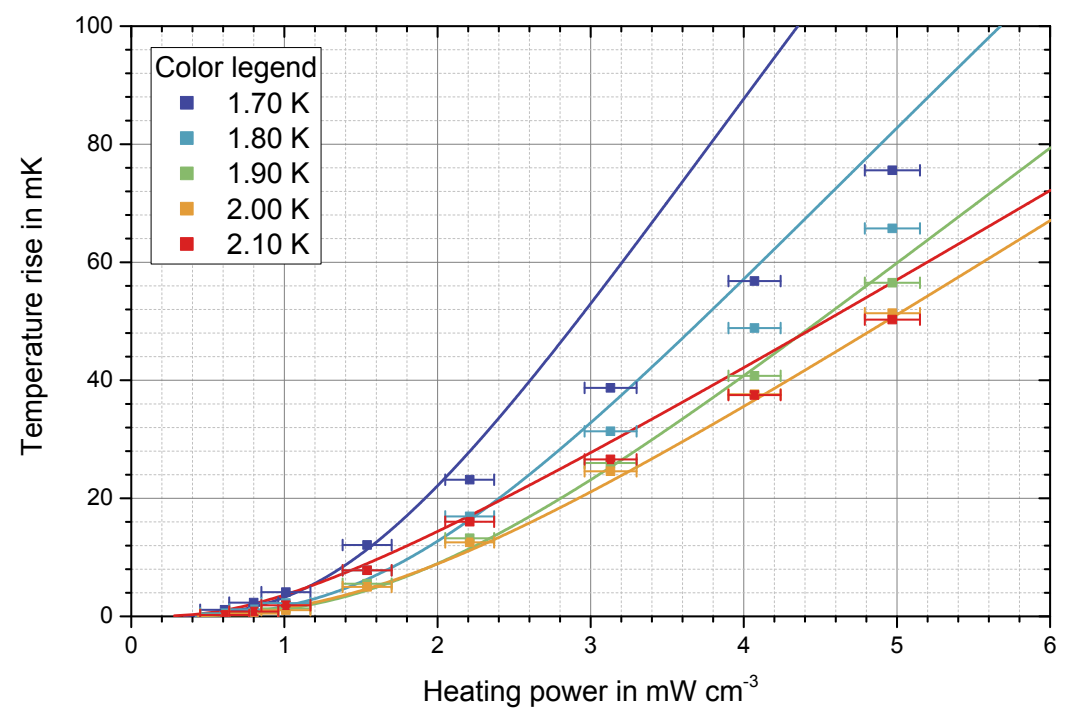

Figure 6.15. The two channel model and the experimental data for a mechanical pressure of $50 \mathrm{MPa}$ in a saturated He bath. Lines are model fits and points are experimental data.

Table 6.5. Fit parameters for the temperature range $1.7 \mathrm{~K}$ to $2.1 \mathrm{~K}$ in saturated $\mathrm{He}$ II and a mechanical sample pressure $50 \mathrm{MPa}$ for the simplified 2 channel model and the original 3 channel model.

\begin{tabular}{l|ccc}
$50 \mathrm{MPa}$ & $\Theta_{1}=\frac{A_{1}}{\frac{1}{l_{1.4}^{3.4}}}$ & $\Theta_{2}=\frac{A_{2} d_{2}^{2}}{l_{2}}$ & $\Theta_{3}=\frac{A_{3} d_{3}^{2}}{l_{3}}$ \\
sat He II & in $\mathrm{m}^{\frac{5.8}{3.4}}$ & ${\text { in } \mathrm{m}^{3}}^{\text {in } \mathrm{m}^{3}}$ \\
\hline \hline 3 Channel model & $1.83 \times 10^{-8}$ & $2.77 \times 10^{-18}$ & $1.01 \times 10^{-15}$ \\
2 Channel model & $1.83 \times 10^{-8}$ & $2.76 \times 10^{-18}$ & -
\end{tabular}

data set at a mechanical pressure of $50 \mathrm{MPa}$ in saturated helium is used as input for the fitting routine of the model. Comparing the result of the simplified model with the three channel model (compare Figure 6.11) no difference can be seen. Table 6.5 lists the fit parameters that were obtained by the two models.

The simplified model was also used to fit the data set for one He bath temperature individually. Figure 6.16 shows the results of this fitting process. Again no change is visible between the simplified two channel model compared to the original three channel model (compared Figure 6.12). The fit parameters obtained for each $\mathrm{He}$ bath temperature are given in Table 6.6.

From Table 6.6 it can be seen that channel 1 does not show any significant change in the fit parameter value. The same can be said for the fit parameter 


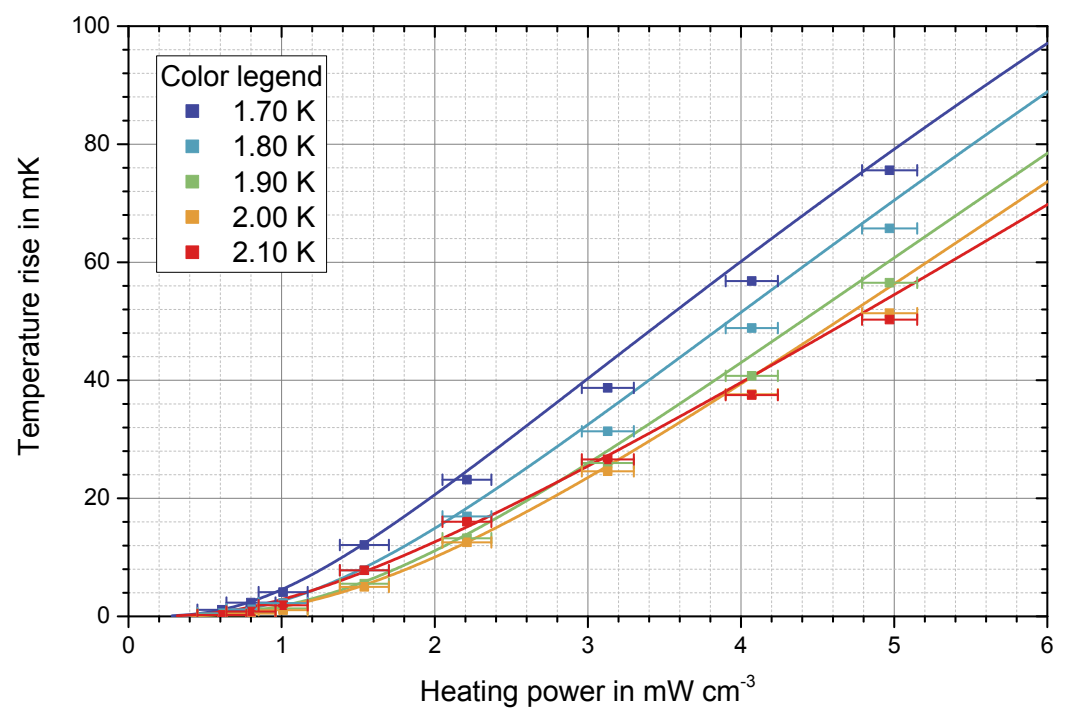

Figure 6.16. The model results and experimental data when each He bath temperature is fitted individually. Lines are results from the model, points are experimental data.

for channel 2 for He bath temperatures above $1.9 \mathrm{~K}$. As expected, for He bath temperatures lower than $1.9 \mathrm{~K}$ the fit parameter value is actually closer to the fit parameter value obtained from the three channel model for channel 3 .

From the discussion of the steady-state measurement results it can thus be said that at least in the heating power range investigated in this work the channel created by the third layer of the polyimide insulation can be neglected and considered to be at the same temperature as the He bath. The two remaining channels located on the small face and the broad face of the cable, respectively, play a very important role at different applied heating powers. Channel 1, located on the small face, is very important at low heating power but decreases in importance with increasing heating power. Eventually channel 2, located on the broad face is dominant in the cooling of the sample in steady-state conditions at a heating power above $2 \mathrm{~mW} \mathrm{~cm}^{-3}$. 
Table 6.6. Fit parameters for the bath temperatures $1.7 \mathrm{~K}, 1.8 \mathrm{~K}, 1.9 \mathrm{~K}, 2.0 \mathrm{~K}$ and $2.1 \mathrm{~K}$ in saturated He II and a mechanical sample pressure $50 \mathrm{MPa}$ for the simplified 2 channel model and the original 3 channel model.

\begin{tabular}{lc|ccc}
$50 \mathrm{MPa}$ & & $\Theta_{1}=\frac{A_{1}}{\frac{1}{l^{3.4}}}$ & $\Theta_{2}=\frac{A_{2} d_{2}^{2}}{l_{2}}$ & $\Theta_{3}=\frac{A_{3} d_{3}^{2}}{l_{3}}$ \\
sat He II & & in $\mathrm{m}^{\frac{5.8}{3.4}}$ & in $^{3}$ & in $^{3}$ \\
\hline \hline 3 Channel model & $1.7 \mathrm{~K}$ & $1.51 \times 10^{-8}$ & $2.64 \times 10^{-15}$ & $9.94 \times 10^{-18}$ \\
2 Channel model & $1.7 \mathrm{~K}$ & $1.49 \times 10^{-8}$ & $1.01 \times 10^{-17}$ & - \\
\hline 3 Channel model & $1.8 \mathrm{~K}$ & $1.55 \times 10^{-8}$ & $5.07 \times 10^{-16}$ & $5.17 \times 10^{-18}$ \\
2 Channel model & $1.8 \mathrm{~K}$ & $1.53 \times 10^{-8}$ & $5.19 \times 10^{-18}$ & - \\
\hline 3 Channel model & $1.9 \mathrm{~K}$ & $1.61 \times 10^{-8}$ & $3.30 \times 10^{-18}$ & $7.30 \times 10^{-16}$ \\
2 Channel model & $1.9 \mathrm{~K}$ & $1.60 \times 10^{-8}$ & $3.33 \times 10^{-18}$ & - \\
\hline 3 Channel model & $2.0 \mathrm{~K}$ & $1.76 \times 10^{-8}$ & $2.49 \times 10^{-18}$ & $6.39 \times 10^{-16}$ \\
2 Channel model & $2.0 \mathrm{~K}$ & $1.75 \times 10^{-8}$ & $2.51 \times 10^{-18}$ & - \\
\hline 3 Channel model & $2.1 \mathrm{~K}$ & $2.09 \times 10^{-8}$ & $2.76 \times 10^{-18}$ & $4.79 \times 10^{-16}$ \\
2 Channel model & $2.1 \mathrm{~K}$ & $2.08 \times 10^{-8}$ & $2.76 \times 10^{-18}$ & -
\end{tabular}

\subsection{Transient Results}

The second fit parameter of the data analysis, the time constant, is basically the product of the thermal resistance $R$ and the heat capacity $C$. The thermal resistance $R$ is the ratio of the temperature rise $\Delta T_{\max }$ in the steady-state and the heating power $Q$. As is obvious from the previous section this resistance is strongly dependent on the He bath temperature and the heating power. It can therefore be expected that also the time constant strongly depends on these parameters.

The time constant for a mechanical sample pressure of $50 \mathrm{MPa}$ in saturated $\mathrm{He}$ II is in the order of $0.4 \mathrm{~s}$ to $2 \mathrm{~s}$ as can be seen in Figure 6.17, where the determined time constant is shown as a function of the He bath temperature. The time constant is increasing with an increasing He bath temperature for an applied heating power higher than $1.54 \mathrm{~mW} \mathrm{~cm}^{-3}$ and reaches a value close to $2 \mathrm{~s}$ at a He bath temperature of $2.1 \mathrm{~K}$. The time constant for a heating power lower than $1.54 \mathrm{~mW} \mathrm{~cm}^{-3}$ shows a constant value of approximately $0.4 \mathrm{~s}$ for He bath temperatures up to $2.0 \mathrm{~K}$. An excursion from this constant value is observed for $2.05 \mathrm{~K}$ and $2.1 \mathrm{~K}$ where only the smallest measured heating power results in a time constant of $0.4 \mathrm{~s}$ and the time constant is increasing for an applied heating power of $0.80 \mathrm{~mW} \mathrm{~cm}^{-3}$ and $1.01 \mathrm{~mW} \mathrm{~cm}^{-3}$.

For a detailed discussion a different graphical presentation of the analysed data is useful. A graphical representation of the time constant against the steady-state temperature difference is advantageous since it includes the general trends of the effective thermal conductivity in the scale of the horizontal axis.

Apart from the data set for a mechanical sample pressure of $50 \mathrm{MPa}$ in a saturated He bath (compare Figure 6.2, in Figure 6.18 three additional data sets with different experimental conditions are included, the raw data sets of these 


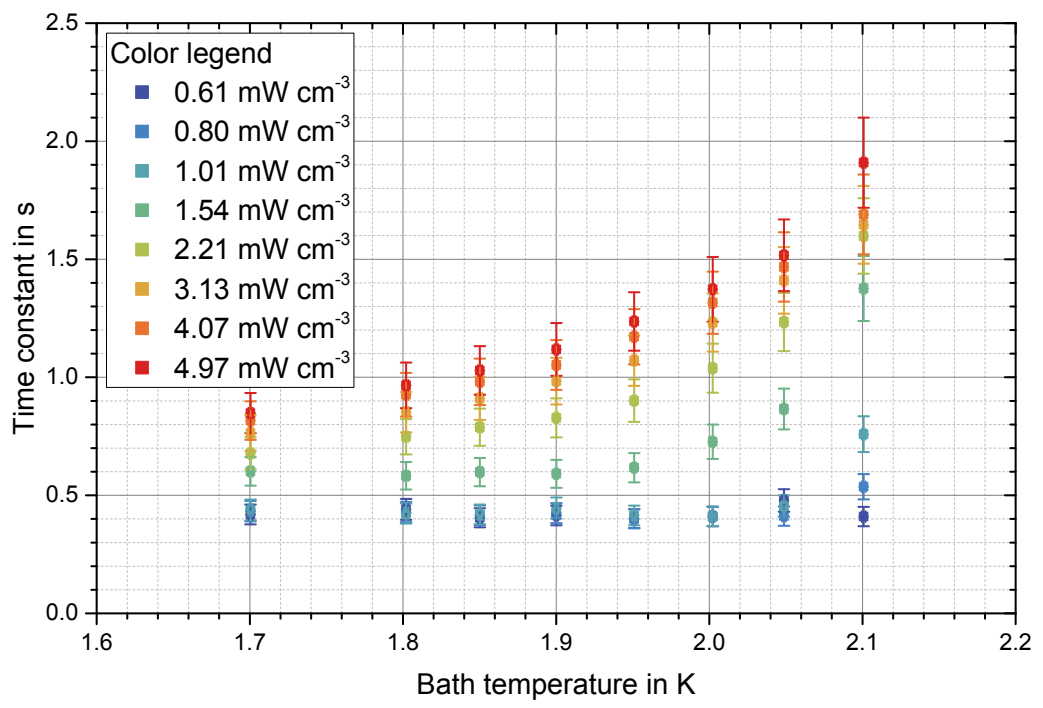

Figure 6.17. Shown is the time constant versus the He bath temperature for a mechanical sample pressure of $50 \mathrm{MPa}$ at saturation conditions. At a He bath temperature of $2.05 \mathrm{~K}$ the time constant at a heating power of $0.61 \mathrm{~mW} \mathrm{~cm}^{-3}$ appears to be an outlier, which is visible throughout the following discussion of the transient results.

measurements can be found in Appendix A

The time constant is shown in Figure 6.18 as a function of the steady-state temperature rise for four different experimental conditions. It includes two different mechanical sample pressures of $50 \mathrm{MPa}$ and $75 \mathrm{MPa}$ at saturation conditions and in pressurised He II. The horizontal axis of the graph is logarithmic in order to improve the visibility of the distinct feature of this graph, where the time constant is a very characteristic function of the steady-state temperature difference. For small steady-state temperature differences the time constant has a constant value of $0.4 \mathrm{~s}$ independent of the He bath temperature nor of the mechanical sample pressure. The transition to a second regime is temperature dependent and in that regime the time constant is monotonically increasing with an increasing steady-state temperature rise. The slope of the functional dependency is again a function of the temperature and the time constant appears to be asymptotically reaching a temperature dependent end value, a fact that is slightly obscured by the logarithmic horizontal axis.

\subsubsection{Effective Volumetric Heat Capacity}

In the single exponential function, that is derived in Section 3.4, the time constant is the product of the effective thermal resistance calculated from the steady-state 


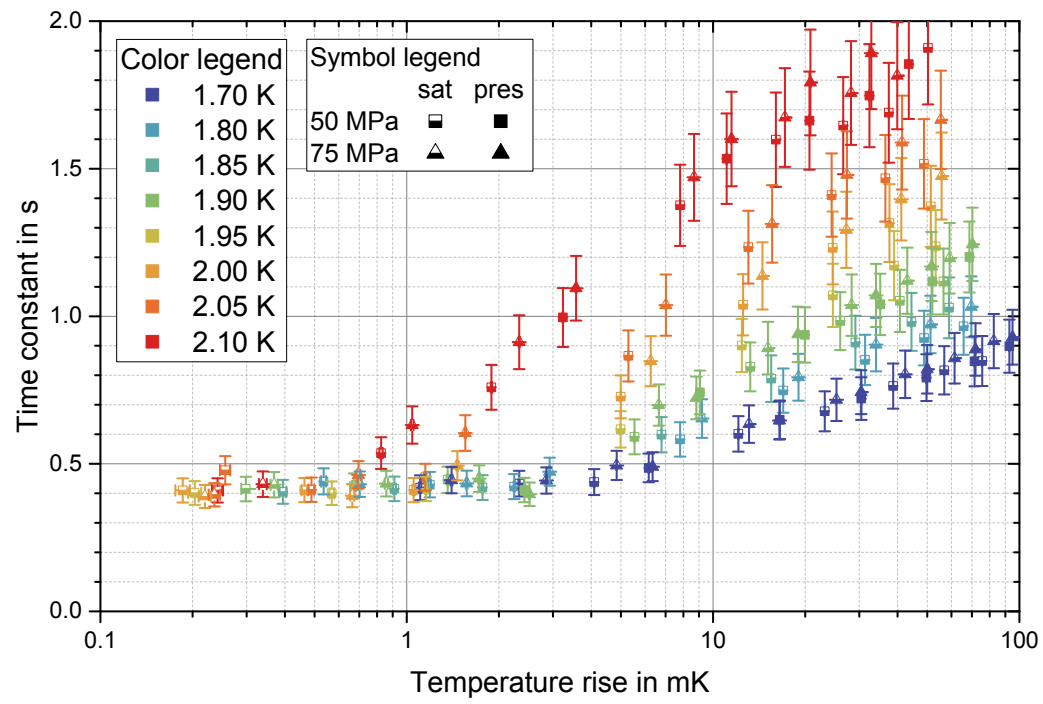

Figure 6.18. The time constant versus the steady-state temperature difference for different experimental conditions including different mechanical sample pressures and measurements in saturated and pressurised He II for the full range of heating power. The separation of the graph into two distinct ranges is immediately visible. A first range, which is visible as a constant plateau of the time constant for small temperature differences independent of the He bath temperature, and a second range with rising time constant and a dependency on the He bath temperature and the temperature difference.

temperature difference and the volumetric heat capacity of the sample. In this relation the effective thermal conductivity and the volumetric heat capacity are assumed to be temperature independent. This functional dependency is used to derive the effective volumetric heat capacity from the time constant and thermal resistance data.

Figure 6.19 shows the effective volumetric heat capacity versus the steady-state temperature rise, where the effective heat capacity results from the above mentioned calculation. The figure shows the effective volumetric heat capacity to be strongly dependent on the steady-state temperature difference and as such on the heating power as well as a small dependency on the He bath temperature.

The first dependency is physically unreasonable and strongly indicates that the steady-state effective thermal conductivity that is used to calculate the effective volumetric heat capacity is not applicable and instead a transient effective thermal conductivity needs to be introduced. The reason for the introduction of a transient effective thermal conductivity is also physically sound when the strong temperature and temperature gradient dependencies of the effective thermal conductivity of $\mathrm{He}$ II is recalled (see Section 2.3.2. 


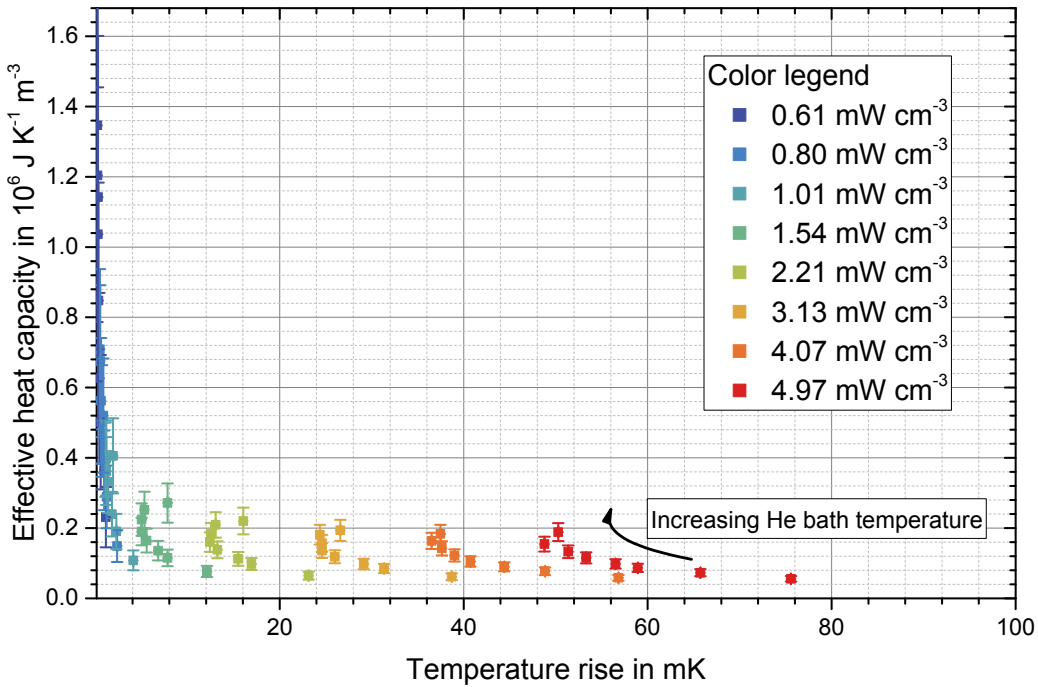

Figure 6.19. The effective volumetric heat capacity versus the steady-state temperature difference for a mechanical sample pressure of $50 \mathrm{MPa}$ in saturated He II. Within one heating power the He bath temperature is varied between $1.7 \mathrm{~K}$ and $2.1 \mathrm{~K}$. The arrow indicates the increasing He bath temperature within a given heating power.

The dependency on the He bath temperature is physically valid since the heat capacity of He is monotonically increasing with an increasing temperature in the investigated temperature range. This behaviour is also found in the order of the measurement points for a given applied heating power, which is indicated by the arrow in Figure 6.19.

\subsubsection{Transient Effective Thermal Conductivity}

It is known from the previous steady-state analysis that the turbulent regime in channel 1 is dominating the overall effective thermal conductivity at low heating power and from Figure 5.2 it is also known that the effective thermal conductivity of channel 1 is decreasing drastically as the temperature gradient increases. Based on this reasoning only the data points obtained with higher heating power need to be considered, because here the laminar heat transfer path is dominant, which does not have a temperature gradient dependency. In this case the effective thermal conductivity is only changing at the beginning of the heating process but for the largest part it will be constant because it is determined by the laminar heat transfer regime.

Furthermore, the heat capacity is composed of two contributions: Namely the cable and the helium that fills the voids between the strands. The effective heat 


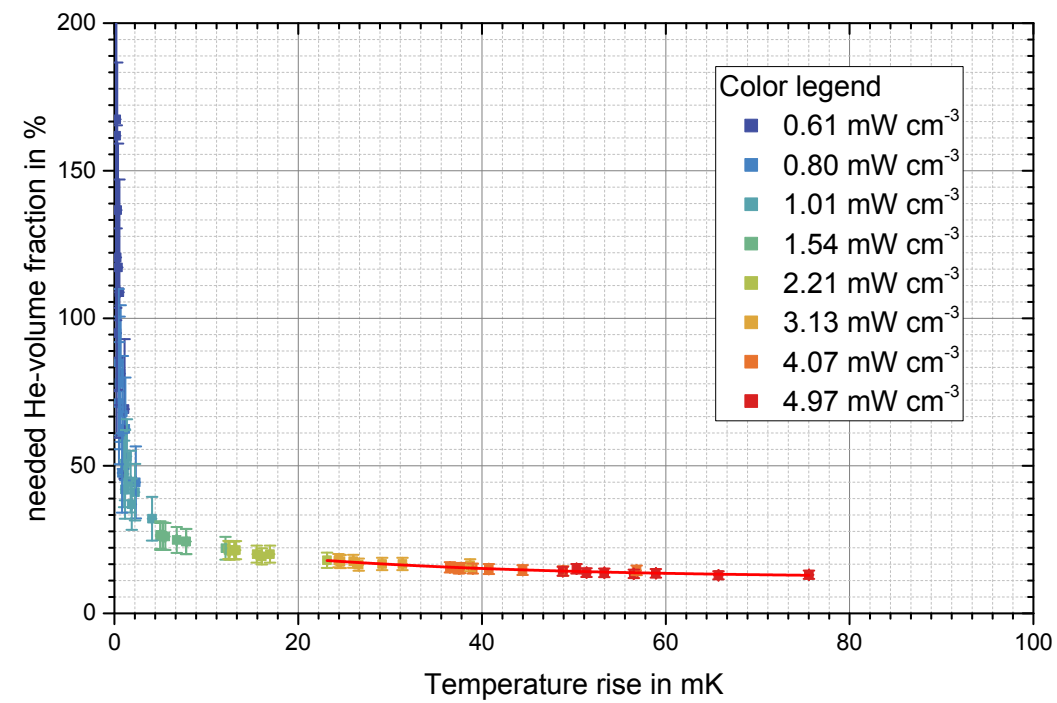

Figure 6.20. The He volume fraction in the sample needed in order to reach a volumetric heat capacity as shown in Figure 6.19 as a function of the steady-state temperature difference for a mechanical sample pressure of $50 \mathrm{MPa}$ at saturation conditions. The red line is a fit to obtain the effective He void fraction in the sample.

capacity of the sample can thus be expressed as:

$$
c_{\text {sample }}=\text { He-volume fraction } c_{H e}+(1-\text { He-volume fraction }) c_{\text {cable }}
$$

$$
\text { He-volume fraction }=\frac{c_{\text {sample }}-c_{\text {cable }}}{c_{H e}-c_{\text {cable }}} .
$$

The helium volume fraction based on Equation (6.3) was evaluated for all data points in Figure 6.19 and presented as a function of the temperature rise in Figure 6.20.

As mentioned above the specific heat should be derived from the high-power limit. Therefore an exponential fit was made in Figure 6.20 through the data points above a temperature rise of $20 \mathrm{mK}$. This leads to a helium volume fraction of about $12 \%$, which is close to the void fraction of about $10 \%$ as calculated in Section 5.2.1 Based on this He volume fraction and the resulting effective heat capacity it is now possible to investigate the transient effective thermal conductivity.

Using the fit value of $12 \%$ He content the total volumetric heat capacity for each data point is calculated and based on the measured time constant the transient effective thermal conductivity is calculated. Figure 6.21 shows the transient effective thermal conductivity versus the He bath temperature. This plot should be 


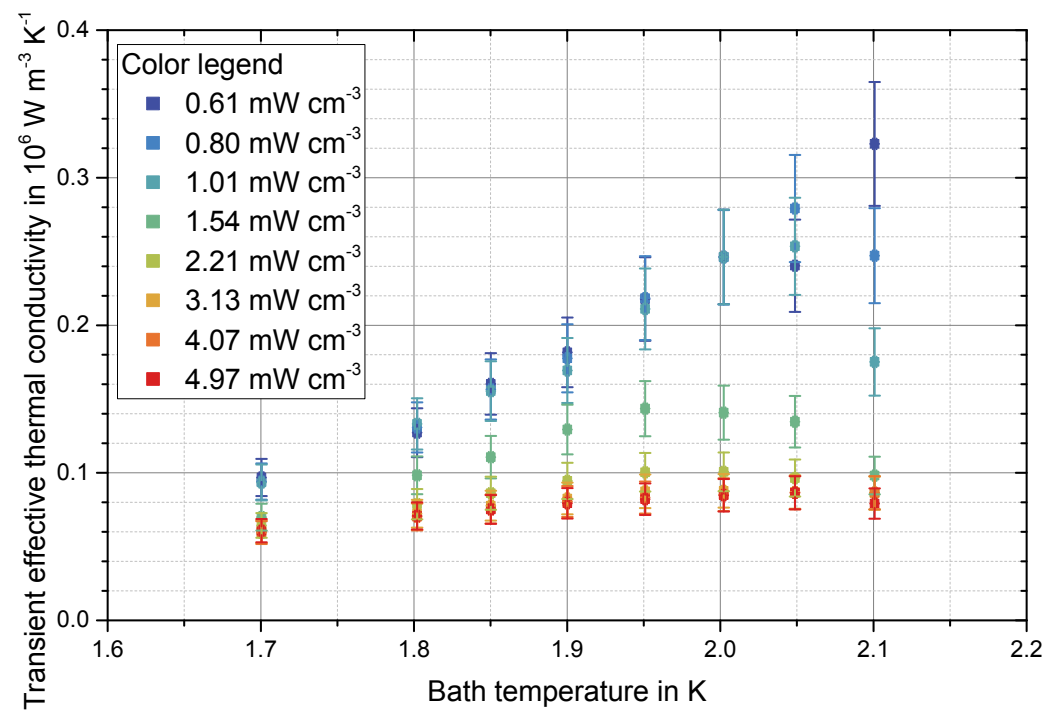

Figure 6.21. The transient effective thermal conductivity versus the He bath temperature. The transient effective thermal conductivity is calculated from the real effective heat capacity and the time constant. As mentioned before the lowest heating power at $2.0 \mathrm{~K}$ appears to be an outlier.

compared with Figure 6.4 in which the difference in vertical scale should be noted. At low heating power the transient effective thermal conductivity is significantly smaller than the steady-state value, whereas at the highest heating power only a small difference can be noticed. This can be explained by the fact that in the transient process the heating results in a local temperature gradient larger than the temperature gradient in steady-state conditions. This behaviour was also found by S.W. van Sciver who investigated the transient heat transfer in a channel filled with He II 74 .

As indicated by Figures 5.2 and 5.3 these increased thermal gradients have a dramatic effect on the effective thermal conductivity in the turbulent regime but do not affect the effective thermal conductivity in the laminar regime. As discussed in the first part of this chapter, at low heating power the heat transfer in steady-state is dominated by channel 1, which is in the turbulent regime. In the transient process the effective thermal conductivity of channel 1 is strongly affected by the steep local gradients so that it may drop below the effective thermal conductivity of channel 2 that is hardly affected by the increased temperature gradients since it is in the laminar regime. This is illustrated in the left schematic of Figure 6.22, note the logarithmic vertical scale in contrast to the linear scale of Figures 5.2 and 5.3 . In Figure 6.22 it is important to note that channel 1 has a larger diameter than channel 2 , because of the buckling of the polyimide 


\section{Results}

insulation, see Section 5.2.1. Since the effective thermal conductivity in the laminar regime scales with the diameter squared this has a large effect on the relative contributions of both channels to the overall effective thermal conductivity. As a result of the local gradients the effective thermal conductivity of the combination of both channels in parallel is significantly reduced and now is dominated by channel 2. And since channel 2 is still in the laminar regime the He bath temperature has a strong effect on the transient effective thermal conductivity, as can be seen in Figure 6.21 at low heating power.

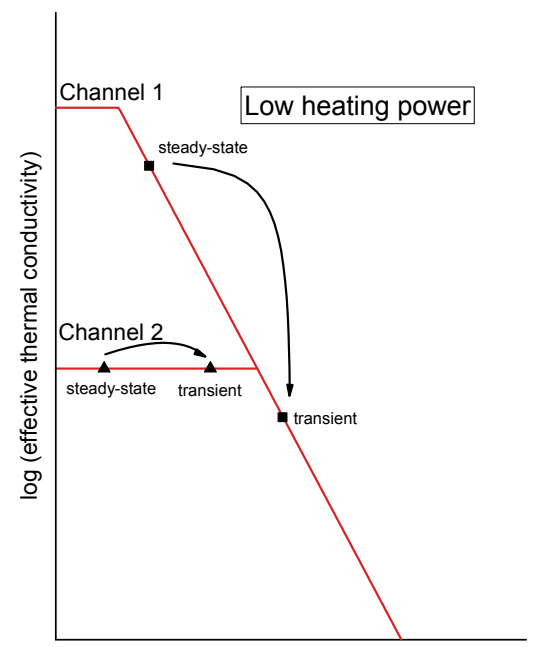

$\log$ (temperature gradient)

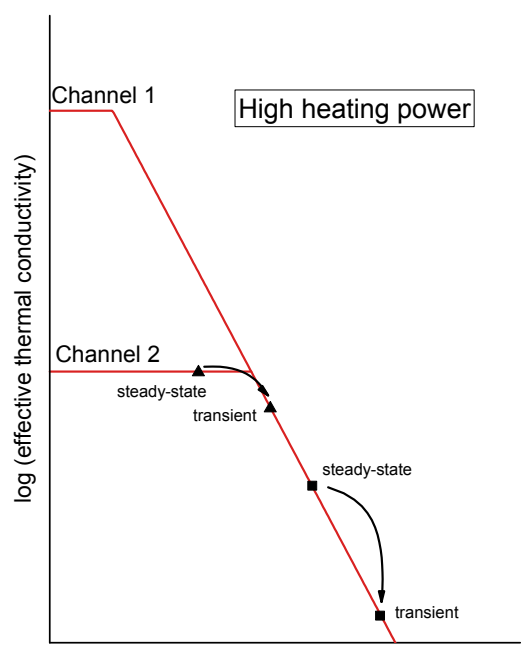

$\log$ (temperature gradient)

Figure 6.22. Illustration of how the effective thermal conductivity in channel 1 and channel 2 is affected by the local temperature gradients during the transient phase of the measurement for a low applied heating power (left) and for a high applied heating power (right).

In case of a high heating power the situation is different, which is illustrated in Figure 6.22 on the right. In steady-state a large gradient is present, which causes the effective thermal conductivity of channel 1 to be below that of channel 2. The overall effective thermal conductivity in steady-state is thus dominated by channel 2 and it is significantly lower than the steady-state overall effective thermal conductivity at low heating power (see Figure 6.4). When heating power is applied the local gradients will move the effective thermal conductivity of channel 1 even further down, whereas the temperature gradient causes the effective thermal conductivity of channel 2 to be just at the transition between the laminar regime and the turbulent regime, where the effect of the He bath temperature is much less severe.

To illustrate how the effective thermal conductivity in steady-state and transient conditions is affected by the temperature gradient Figure 6.23 shows the ratio of 


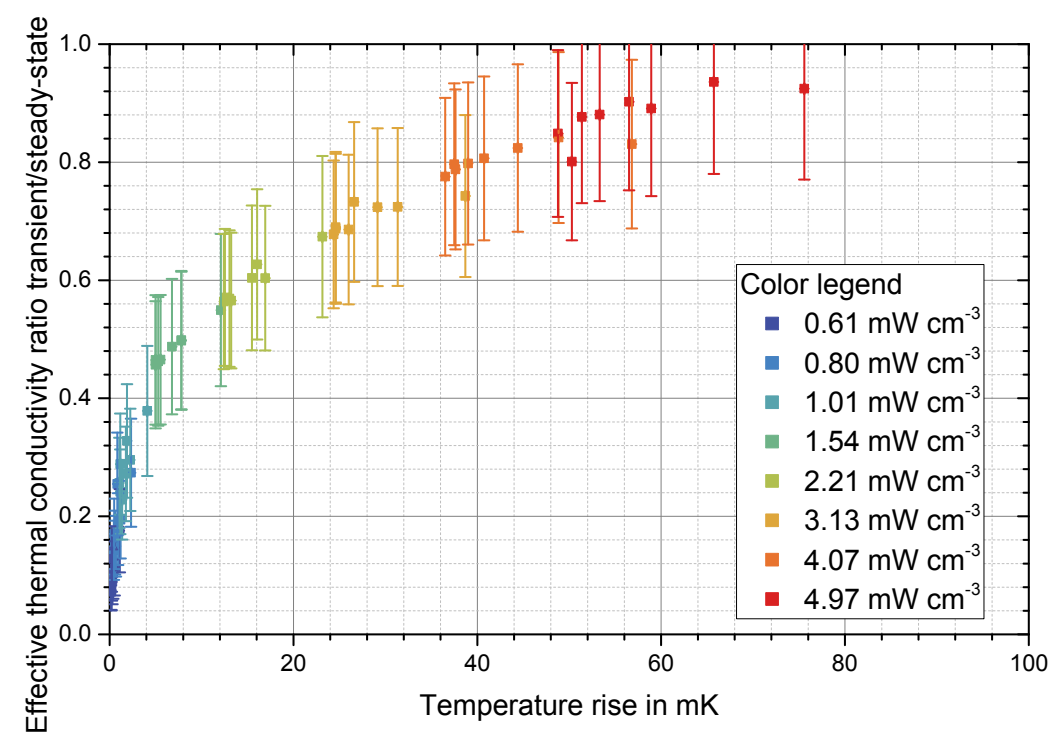

Figure 6.23. The ratio of transient effective thermal conductivity to steady-state effective thermal conductivity versus the steady-state temperature difference.

the two thermal conductivities $\frac{k_{\text {transient }}}{k_{\text {steady-state }}}$ versus the steady-state temperature rise. The transient effective thermal conductivity is always smaller than the steady-state effective thermal conductivity as can be seen in Figure 6.23. For high heating power the effective thermal conductivities in steady-state and in transient state both show an increasingly similar behaviour and the ratio approaches a value of 1 for a high applied heating power. Based on the before mentioned reasoning the transient effective thermal conductivity is always smaller than the steady-state effective thermal conductivity and therefore the ratio should asymptotically reach a value of 1 .

\subsubsection{Concluding Remarks}

Comparing the obtained time constants with the time for the development of turbulence as they were published by W.F. Vinen a non-negligible discrepancy is visible [75]. This discrepancy can be explained when taking into account the conditions W.F. Vinen gives for his experiments. In his work the time to develop turbulence under the conditions that the He volume is small and the specific heat capacity can be neglected. This condition is not full-filled in this work where the specific heat capacity is not negligible and thus plays an important role in the time to reach steady-state conditions. 



\section{Conclusion}

In this work the steady-state and transient cooling of a polyimide insulated superconducting Rutherford cable stack is experimentally investigated and a model for the heat transfer is developed. It was found that the temperature evolution of the sample can be described very well using a single exponential function. From the function two parameters are obtained, the steady-state temperature difference and the characteristic time for the sample to reach $\approx 63 \%$ of the steady-state temperature difference.

In the discussion of the experimental results it was found that the steady-state temperature difference is a strong function of the He bath temperature and of the heating power. It does not show a single proportionality to the applied heating power but shows two distinct ranges. For low heating power it is found that the steady-state temperature difference and the heating power have a dependency that indicates that in this range the heat transfer is dominated by the turbulent heat transfer regime in He II. For higher applied heating power it was found that the steady-state temperature difference and the heating power have an almost linear dependency indicative for He II in the laminar heat transfer regime. As a conclusion it can be said that at least two different heat transfer paths with He II are linking the superconducting Rutherford cable with the He II bath outside of the sample.

Based on these observations a model was developed which uses the geometric parameter of the channels as fitting parameters. Taking into account the pattern of the three polyimide layers of the insulation it is first concluded that overall three channels link the sample with the He bath. One channel is situated on the small face of the sample, the other two channels are in series and have very different geometries.

Using the model different experimental runs are modelled and it is found that the model fits the steady-state temperature difference data very well for a single constant He bath temperature. If the fitting range is extended by varying the He bath temperature it is found that the model fits the data well for He bath temperatures higher than $1.9 \mathrm{~K}$ and a deviation for lower He bath temperatures is found. The deviation could be caused by the way the effective thermal conductivity is modelled with the direct transition approach, which underestimates the effective thermal conductivity in the transition between the laminar and the turbulent heat transfer regime.

To explore the influence of the temperature on the fitting results further individual fits of the different temperatures are made and it is found that the third channel in the model, which is located on the broad face of the cable, can be assumed to be at the He bath temperature and therefore can be neglected. The simplified model gives similar results while showing a much more consistent geometry factor for the 


\section{Conclusion}

different scenarios.

In the study different parameters were varied in the experiment to investigate their influence on the cooling of the sample. The He bath was varied between saturated and sub-cooled conditions in the temperature range $1.7 \mathrm{~K}$ to $2.1 \mathrm{~K}$. It was found that the steady-state temperature difference is slightly higher for measurements in sub-cooled He II compared to saturated He II. This is consistent with theory, which predicts a decrease of the effective thermal conductivity between $4 \%$ to $8 \%$ when increasing the He bath pressure to $0.1 \mathrm{MPa}$ depending on the heat transfer regime.

Two mechanical pressures were investigated to evaluate the influence of different pressures on the He II cooling performance. No definitive statement can be made concerning the influence of the mechanical pressure on the heat transfer since the reproducibility of the experiment is in the same range as the observed difference between the two mechanical pressures $50 \mathrm{MPa}$ and $75 \mathrm{MPa}$.

The second parameter in the temperature evolution of the sample is the characteristic time. The time constant is in the range of $0.4 \mathrm{~s}$ to $2 \mathrm{~s}$. For a given He bath temperature it increases with increasing applied heating power. For a given heating power the time constant is found to be increasing with an increasing $\mathrm{He}$ bath temperature for high heating powers. In the case of heating powers lower than $1.5 \mathrm{~mW} \mathrm{~cm}^{-3}$ the time constant has a constant value of $0.4 \mathrm{~s}$ independent of the heating power with the exception of bath temperatures higher than $2.00 \mathrm{~K}$ where the same behaviour as for high heating powers is observed.

The analysis of the time constant reveals that the effective thermal conductivity of the helium channels is a strong function of the elapsed time. It is found that the effective thermal conductivity in the transient phase is lower than the effective thermal conductivity in steady-state conditions. This is largely attributed to the presence of a high temperature gradient plug in the turbulent He channel. This plug reduces the effective thermal conductivity in the turbulent phase and can explain the low transient effective thermal conductivity. The laminar heat transfer regime does not show any dependency on the temperature gradient. The temperature gradient plug is dominant for low heating powers since in this range the turbulent channel is the main heat transfer path and thus the effect of the plug is very visible here. For higher heating powers this blockage effect of the temperature gradient is still visible but not dominant anymore since in this range the laminar He channel combination is the main heat transfer path.

Comparing the measurement results obtained in this work with other experimental data (from Granieri [52]) on the same insulation pattern several differences can be observed. First of all it is important to note that two different experimental approaches are compared. The approach taken by Granieri is to manufacture a normal conducting cable with the same geometry as the superconducting Rutherford cable. This mock-up cable is then insulated by hand using the same pattern as in the cable of the present study. Several of these cables are then stacked to create a sample. One of the cables is instrumented with temperature sensors to measure the temperature of the sample. A thermocouple is used as temperature sensor and heat is generated in the sample by Joule heating of the transport current in the sample. 
In this work a superconducting cable is used, which is heated using AC loss. The cable is taken from the machine that also insulates the cable for the real magnet. Hence the insulation of the superconducting cable used in this study is the same as in the LHC magnets. The temperature sensor in this study is a Cernox ${ }^{T M}$ sensor.

When comparing the measurement results a few observations can be made. The comparison is possible for the data set of $50 \mathrm{MPa}$ mechanical pressure in a saturated He bath at $1.9 \mathrm{~K}$. The heating power necessary to reach the same temperature difference is about $200 \%$ higher for the measurement of Granieri compared to this work. Some variations can be explained by the limited accuracy and weak reproducibility of the here presented experiments but these errors do not account for the total difference between the two different experiments. The difference between the two measurements indicates that the thermal link between the sample and the He bath is much better in the experiments by Granieri compared to this study. The reason for the different thermal link can be related to the different sample construction. The polyimide insulation when prepared by hand is much less consistent and is wrapped looser when compared to the cable insulation prepared by a dedicated machine.

Unfortunately no additional comparisons could be made since in other studies the insulation pattern is not identical and a comparison therefore not conclusive.

Concerning the transient results this work shows the first detailed results for this case of magnet operation at the LHC.

In this work a detailed look is taken at the cooling of the superconducting magnets used in the LHC and it contributes to the knowledge base needed for the safe operation of LHC magnets. The LHC is exposed to a range of heat loads over a broad range of durations and the transient heat transfer between the cable and He bath is an important factor in the thermal stability of the magnet. Most important to note is the formation of the temperature gradient plug which limits the heat transfer in the transient case. 



\section{A Measurement Data}

In the main part of the thesis the data set obtained for a mechanical sample pressure of $50 \mathrm{MPa}$ measured in a saturated He bath is discussed. In the steadystate discussion the differences to other conditions are discussed for a He bath temperature of $1.9 \mathrm{~K}$. The data for these other measurement conditions are given here. For each experimental condition first the steady-state temperature difference between sample and He bath is shown, followed with the time constant for the same experimental conditions. The experimental conditions are always noted in the plot itself.

The data sets shown here all cover the same temperature range of $1.7 \mathrm{~K}$ to $2.1 \mathrm{~K}$ but do not include as many intermediate He bath temperatures as the data set obtained with $50 \mathrm{MPa}$ in a saturated He bath. The data sets in pressurised helium cover only three He bath temperatures and are limited to six different heating powers. The trends and the the conclusions drawn from these data sets remain identical to the conclusions from the main results discussion. 


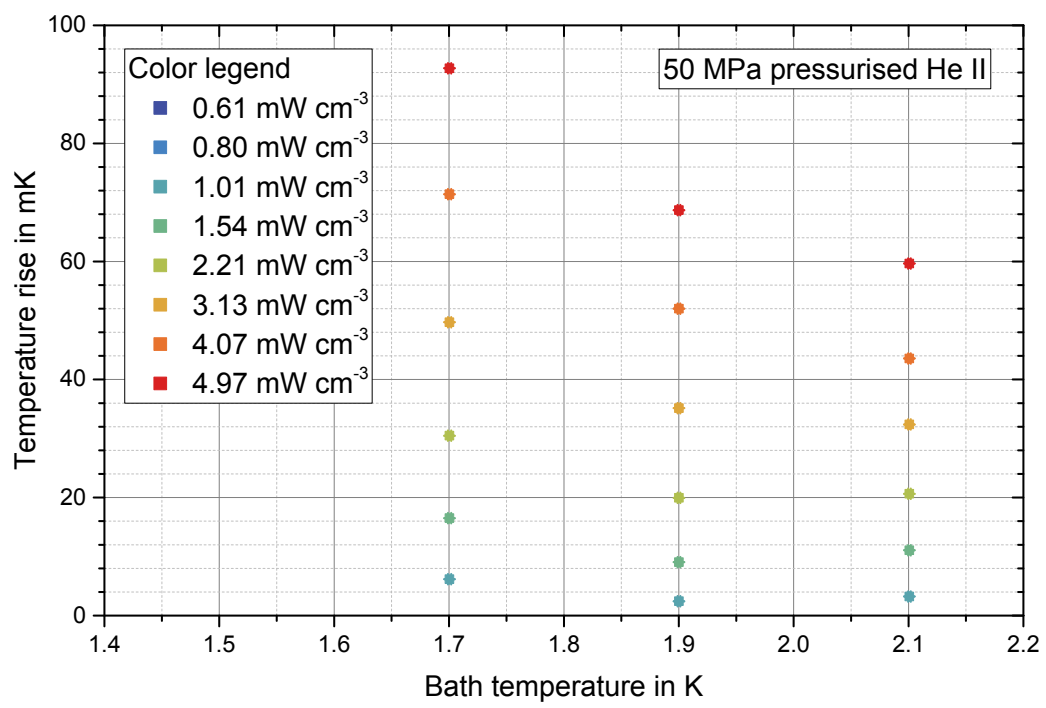

Figure A.1. The temperature rise versus the He bath temperature for a mechanical sample pressure of $50 \mathrm{MPa}$ in pressurised He II.

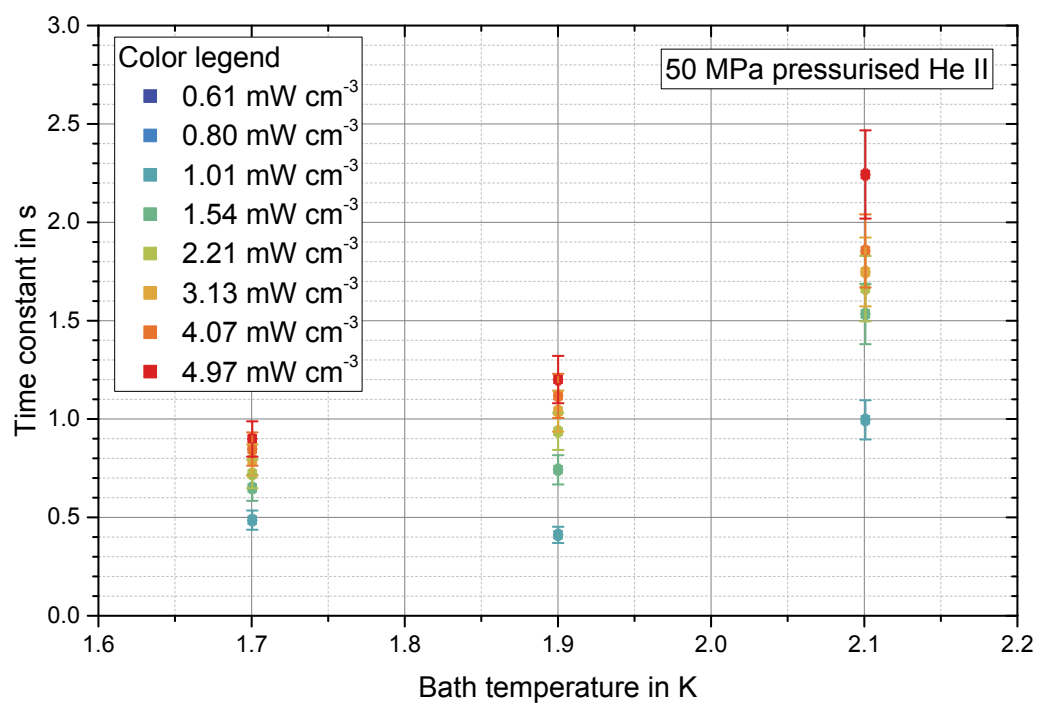

Figure A.2. The time constant versus the He bath temperature for a mechanical sample pressure of $50 \mathrm{MPa}$ in pressurised He II. 


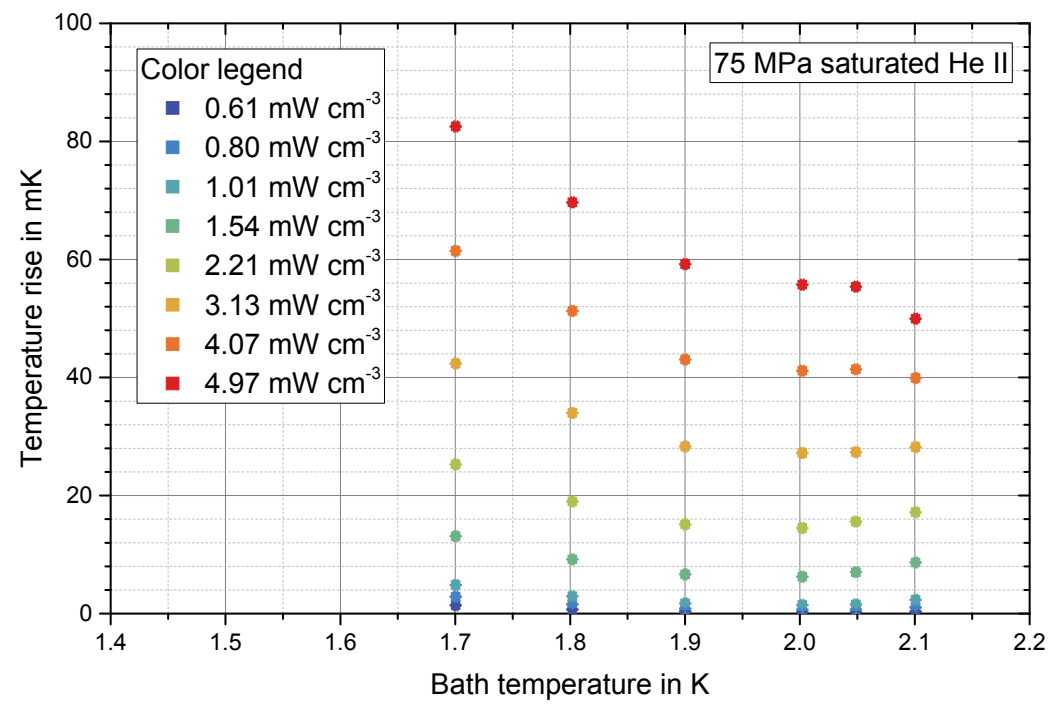

Figure A.3. The temperature rise versus the He bath temperature for a mechanical sample pressure of $75 \mathrm{MPa}$ in saturated He II.

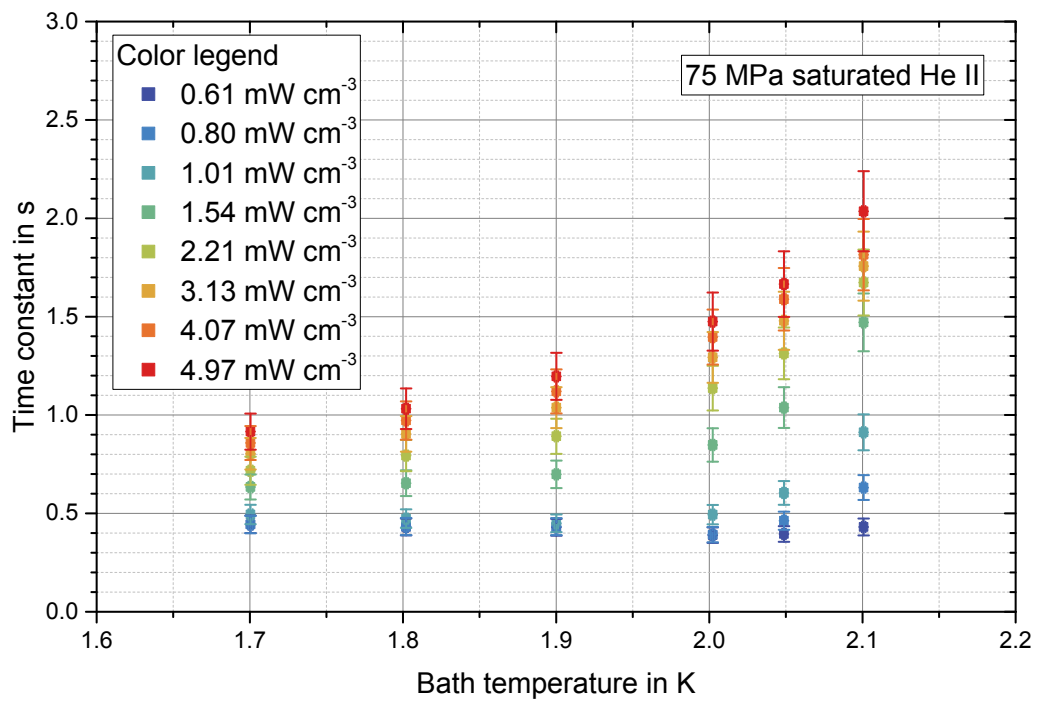

Figure A.4. The time constant versus the He bath temperature for a mechanical sample pressure of $75 \mathrm{MPa}$ in saturated He II. 


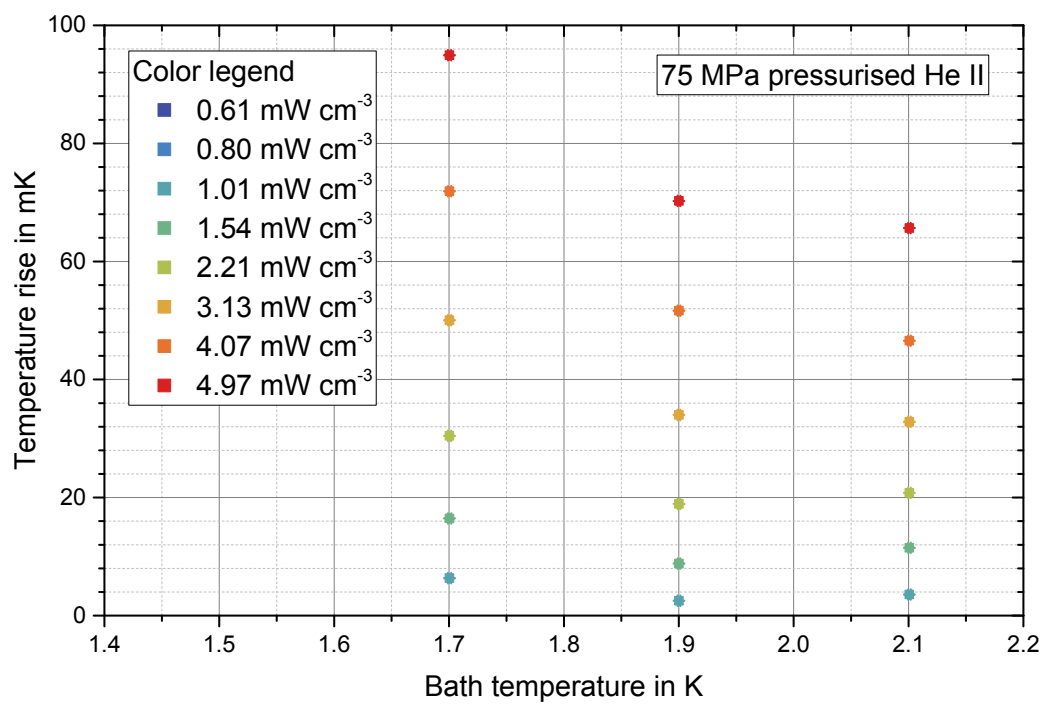

Figure A.5. The temperature rise versus the He bath temperature for a mechanical sample pressure of $75 \mathrm{MPa}$ in pressurised He II.

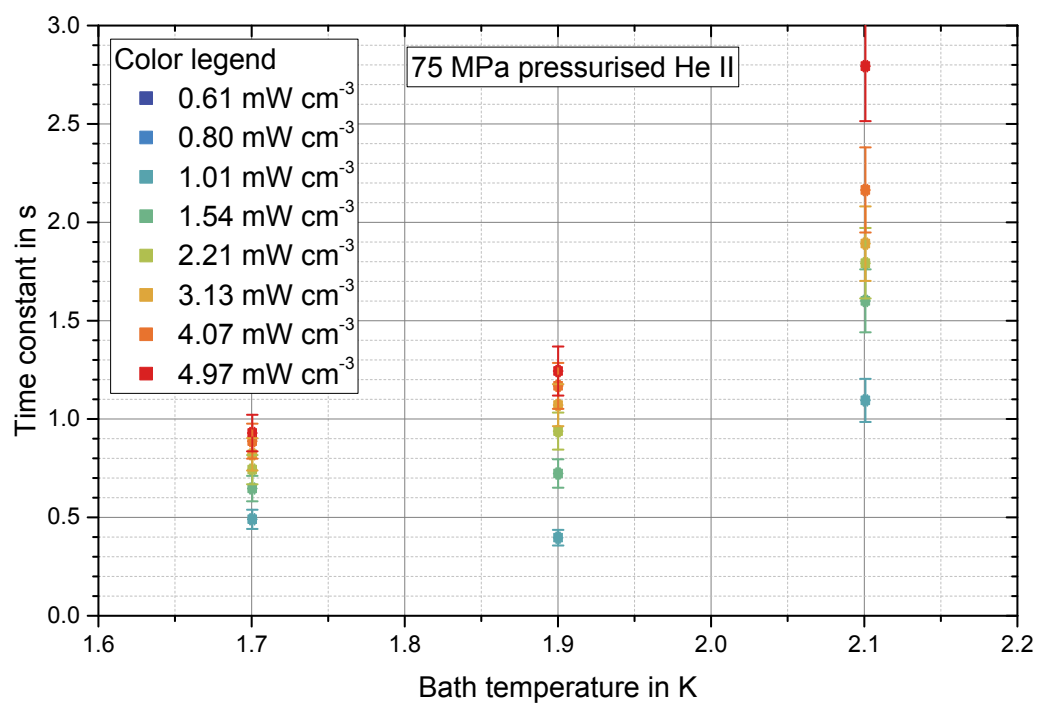

Figure A.6. The time constant versus the He bath temperature for a mechanical sample pressure of $75 \mathrm{MPa}$ in pressurised He II. 


\section{B Two Channel Model Plots}

In the modelling chapter a model for the heat transfer is developed that relies on three helium filled channels to transfer the heat from the heated sample to the He bath. Channel 1 is located on the small face of the sample, the channels 2 and 3 are located on the broad face and are in series with each other. In the discussion of the steady-state results it is found that the model works very well with one peculiar exception. Fitting each He bath temperature individually results in an interesting effect for the geometry factor of the channels 2 and 3. Since a changing geometry is not reasonable at all it was decided to simplify the model and have only channel 2 on the broad face of the cable in the model. This is justified under the assumption that channel 3 is much wider than channel 2 and thus only poses a negligible thermal resistance.

In the discussion of the steady-state results this model is introduced and applied to the full data set to show that this two channel model also fits the measurement data very well and that the differences between the models besides the geometry factor are very small. Here the comparison plots for saturated and pressurised $\mathrm{He}$ II, the two mechanical sample pressures of $50 \mathrm{MPa}$ and $75 \mathrm{MPa}$ are given. Also the plot which shows the composition of the total heat flux is shown. 


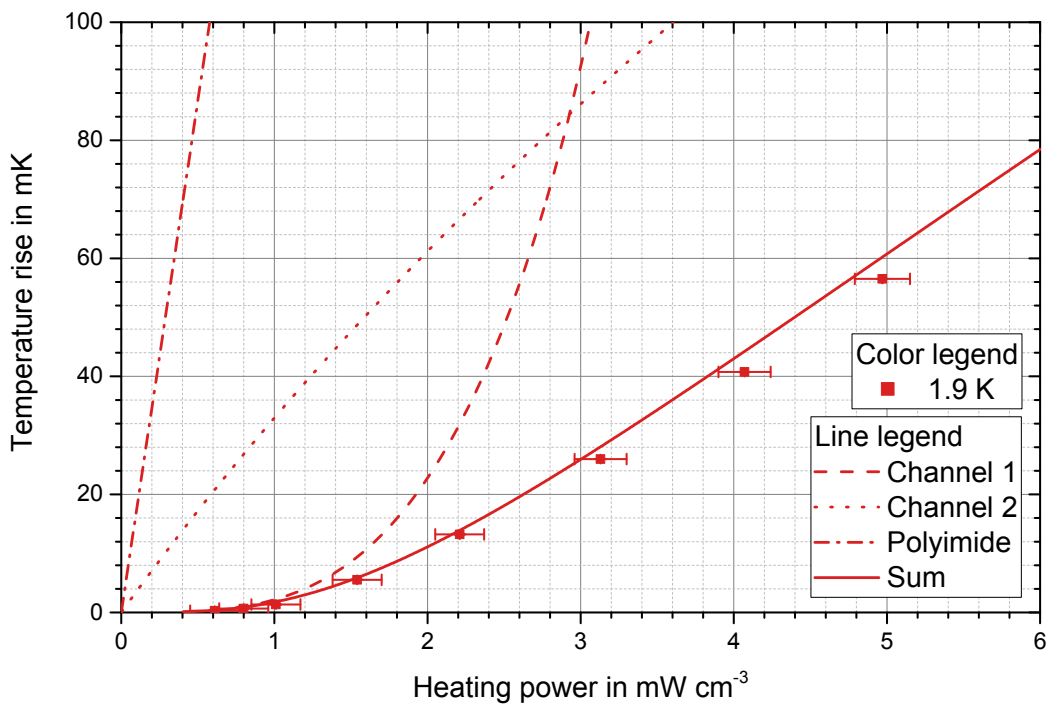

Figure B.1. Graph comparing the results of the $50 \mathrm{MPa}$ measurement runs with the modelled heat flux by the two channel model, when channel 3 is omitted in the model. Also the sum of the heat fluxes is plotted together with the experimental data used for the fitting process.

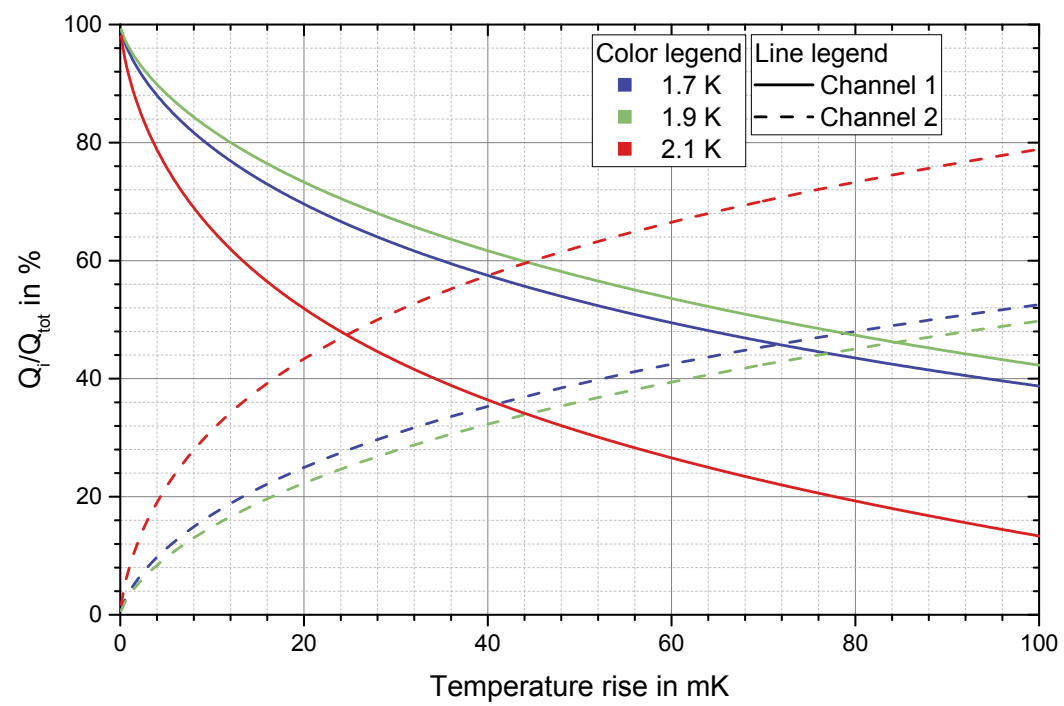

Figure B.2. The calculated percentage of the total heat flux that is transported by channel 1 and channel 2 for three He bath temperatures $1.7 \mathrm{~K}, 1.9 \mathrm{~K}$ and $2.1 \mathrm{~K}$. The missing contribution to $100 \%$ is the solid conduction through the polyimide insulation. 


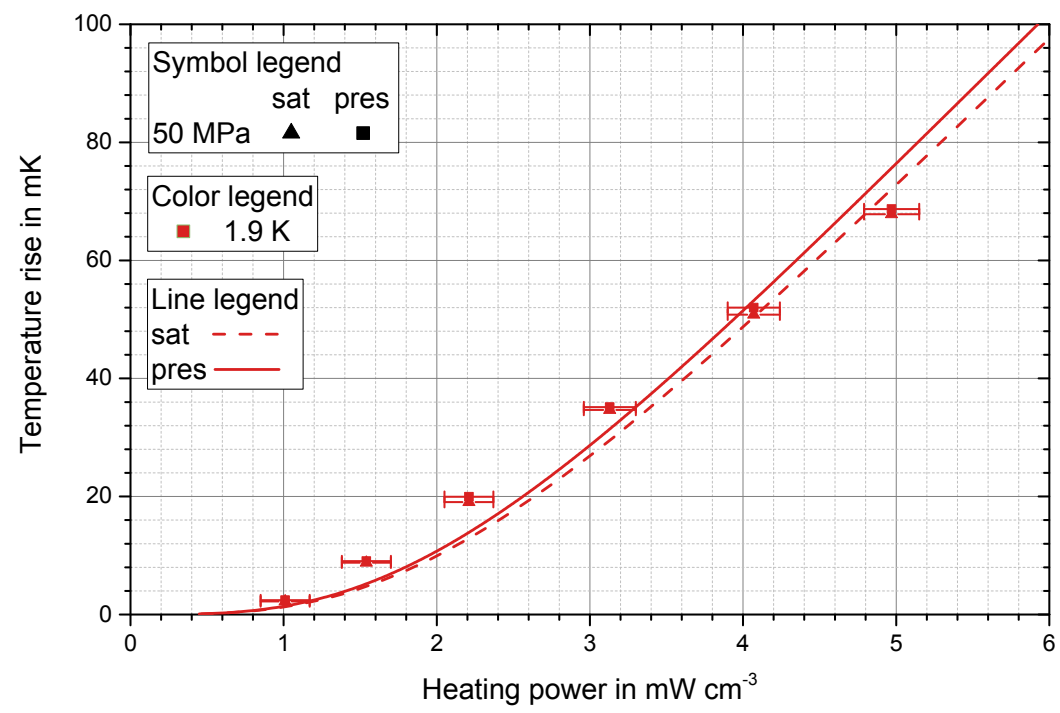

Figure B.3. The model and the measurement data for a mechanical sample pressure of $50 \mathrm{MPa}$ for saturated He II and pressurised He II. Points denote experimental data points and lines are the results of the model.

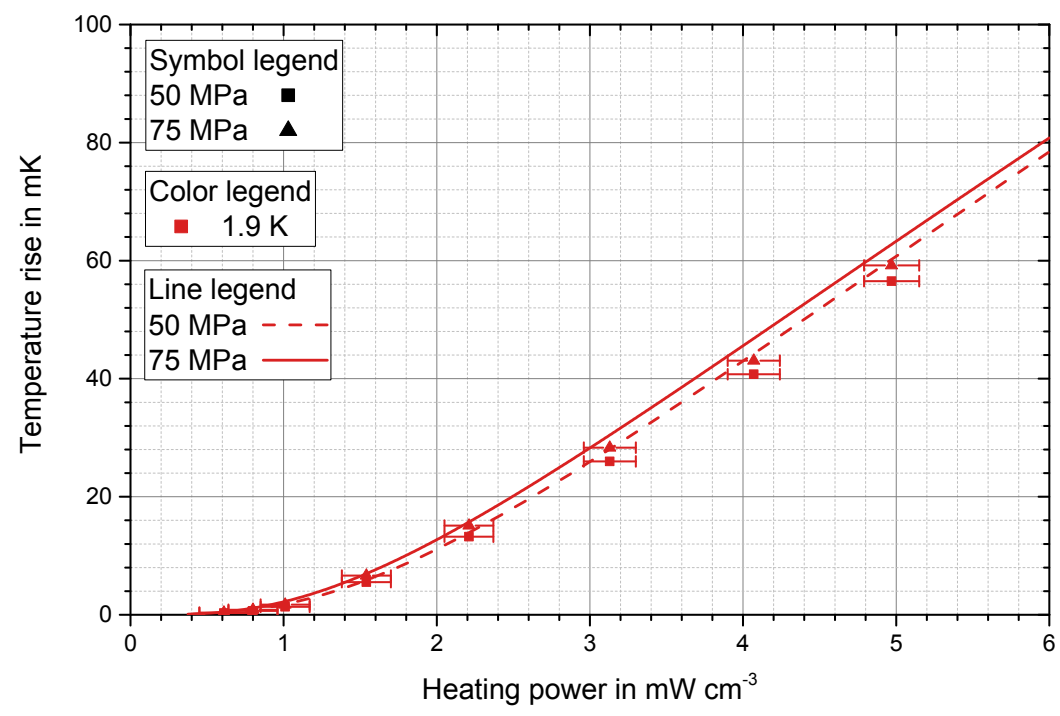

Figure B.4. Comparison of the model and experimental data for $50 \mathrm{MPa}$ and $75 \mathrm{MPa}$ at $1.9 \mathrm{~K}$ under saturation conditions. Lines are model results and points are experimental data points. 



\section{Bibliography}

[1] H. Wiedemann. Particle Accelerator Physics. Springer International Publishing Switzerland, 2015.

[2] P. Vedrine. Large Superconducting Magnet Systems. In R. Bailey, editor, CAS - CERN Accelerator School: Course on Superconductivity for Accelerators. CERN, 2014.

[3] R. de Bruyn Oubuter. Superconductivity: Discoveries During the Early Years of Low Temperature Research at Leiden. IEEE Transactions on Magnetics, $23,1987$.

[4] O.S. Bruening, P. Collier, P. Lebrun, S. Myers, R. Ostojic, J. Poole, and P. Proudlock, editors. LHC Design Report Volume I: The LHC Main Ring. CERN Scientific Information Service, 2004.

[5] L. Rossi and O. Brüning. High Luminosity Large Hadron Collider A description for the European Strategy Preparatory Group. CERN Scientific Information Service, 2012.

[6] K.H. Bennemann and J.B. Ketterson. Superconductivity. Springer-Verlag Berlin Heidelberg, 2008.

[7] S.W. van Sciver. Helium Cryogenics. Springer, 2012.

[8] L. Bottura. A Practical Fit for the Critical Surface of NbTi. IEEE Transactions on Applied Superconductivity, 10:1054 - 1057, 2000.

[9] L. Burnod and J.B. Jeanneret. Beam Losses and Collimation at the LHC: A Quantitative Apporach. LHC Note 167, CERN, 1991.

[10] M.N. Wilson. Superconducting Magnets. Oxford University Press, 1983.

[11] A.P. Verweij. Quenches after LS1. In C. Carli, editor, Proceedings of Chamonix 2012 Workshop on LHC Performance. CERN, 2012.

[12] D. van Delft. Little Cup of Helium, Big Science. Physics Today, 61(3):36 - 42, 2008.

[13] H.K. Onnes. The Liquifaction of Helium. In Koninklijke Nederlandse Akademie von Wetenschappen, Proceedings, volume 11, pages 168 - 185, 1908.

[14] P. Kapitsa. Viscosity of Liquid Helium Below the $\lambda$-Point. Nature, 141:74, 1938. 


\section{BIBLIOGRAPHY}

[15] J.F. Allen and A.D. Misener. Flow of Liquid Helium II. Nature, 141:74-75, 1938.

[16] H.A. Kierstad. Lambda Curve of Liquid $\mathrm{He}^{4}$. Physical Review, 162(1):153 161, October 1967.

[17] J.A. Lipa, D.R. Swanson, J.A. Nissen, and T.C.P. Chui. Lambda Point Experiment in Microgravity. Cryogenics, 34(5):341 - 347, 1994.

[18] O.K. Rice. The Nature of Higher-Order Phase Transitions with Applications to Liquid Helium. Physical Review, 93(6):1161 - 1168, 1954.

[19] J.O. Wilhelm, A.D. Misener, and A.R. Clark. The Viscosity of Liquid Helium. Proceedings of the Royal Society of London, Series A, Mathematical and Physical Sciences, 151:342 - 347, 1935.

[20] W.H. Keesom and A.P. Keesom. On the Heat conductivity of Liquid Helium II. Physica, III:359 - 360, 1936.

[21] L. Landau. Theory of the Superfluidity of Helium II. Physical Review, 60:356 $-358,1941$.

[22] L. Tisza. Sur la theorie des liquides quantiques. Application a l'helium liquide II. J. Phys. Radium, 1:350 - 358, 1940.

[23] R.P. Feynman. Atomic Theory of the Two-Fluid Model of Liquid Helium. Physical Review, 94(2):262 - 277, April 1954.

[24] W.H. Keesom and G.E. MacWood. The Viscosity of Liquid Helium. Physica, $\mathrm{V}: 737-744,1938$.

[25] C.J Gorter and J.H. Mellink. On the Irreversible Processes in Liquid Helium II. Physica, XV:285 - 304, May 1949.

[26] C.J. Gorter. On the Thermodynamics of the Two-Fluid Model of Helium II. Physica, XV:523 - 531, May 1949.

[27] D.V. Osborne. The Rotation of Liquid Helium II. Proceedings of the Royal Society of London, Series A, Mathematical and Physical Sciences, 63:909912, 1950.

[28] L. Onsager. Nuovo cimento. Suppl, 6(2):1949, 1949.

[29] F. London. Superfluids. Wiley, 1954.

[30] R.P. Feynman. Progress in Low Temperature Physics, volume 1, chapter II Application of Quantum Mechanics to Liquid Helium, pages 17 - 53. Elsevier, 1955.

[31] G.A. Williams and R.E. Packard. Photographs of Quantized Vortex Lines in Rotating He II. Physical Review Letters, 33(5):280 - 283, 1974. 
[32] G.P. Bewley, D.P. Lathrop, and K.R. Sreenivasan. Superfluid Helium: Visualization of Quantized Vortices. Nature, 441:588, 2006.

[33] H.E. Hall and W.F. Vinen. The Rotation of Liquid Helium II: I. Experiments on the Propagation of Second Sound in Uniformly Rotating Helium II. Proceedings of the Royal Society of London, Series A, Mathematical and Physical Sciences, 238:204-214, 1956 .

[34] H.E. Hall and W.F. Vinen. The Rotation of Liquid Helium II: II. The Theory of Mutual Friction in Uniformly Rotating Helium II. Proceedings of the Royal Society of London, Series A, Mathematical and Physical Sciences, 238:215234, 1956.

[35] M. Sciacca, D. Jou, and M.S. Mongiovi. Effective Thermal Conductivity of Helium II: From Landau to Gorter-Mellink Regimes. Z. Angew. Math. Phys., $66: 1835$ - 1851, 2015.

[36] D.R. Ladner and J.T. Tough. Helium II Thermal Counterflow at Large Heat Currents: Profound Effects of Geometry. Physical Review B, 17(3):1455 1457, February 1978.

[37] J.D. Henberger and J.T. Tough. Geometric Effects on Superfluid Turbulence: Rectangular, Square and Circular Tubes Compared. Physical Review B, 23(1): 413 - 414, January 1981.

[38] W.F. Vinen. Mutual Friction in a Heat Current in Liquid Helium II. III. Theory of the Mutual Friction. Proceedings of the Royal Society of London, Series A, Mathematical and Physical Sciences, 242(1231):493 - 515, November 1957.

[39] J.T. Tough. Progess in Low Temperature Physics, volume VIII, chapter 3 Superfluid Turbulence. North-Holland Publishing Company, 1982.

[40] D. Jou and M. Sciacca. Quantum Reynolds Number for Superfluid Counterflow Turbulence. Boll. di mat. Pura ed appl., VI:95 - 103, 2013.

[41] A. Sato, M. Maeda, and Y. Kamioka. Steady State Heat Transport in a Channel Containing He II at High Pressures up to 1.5 MPa. AIP Conf. Proc., $710,2004$.

[42] C. Meuris. Heat Transport in Insulation of Cables Cooled by Superfluid Helium. Cryogenics, 31:624-628, 1991.

[43] B. Baudouy, F.-P. Juster, C. Meuris, and L. Vieillard. Steady-State Heat Transfer in He II Through Porous Superconducting Cable Insulation. Advances in Cryogenic Engineering, 41:289 - 296, 1996.

[44] B. Baudouy, A. Boucheffa, M.X. François, and C. Meuris. Thermal Behaviour of Electrical Multilayer Insulation Permeable to Superfluid Helium. Proc. 16. ICEC/ICMC, pages $563-566,1997$. 


\section{BIBLIOGRAPHY}

[45] B. Baudouy. Etude des Transferts de Chaleur dans les Isolations Electriques de Cables Supraconducteurs d'Aimant d'Accelerateur Refroidi par Helium Superfluide. PhD thesis, Universite Pierre et Marie Curie, 1996.

[46] B. Baudouy, M.X. Francois, F.-P. Juster, and C. Meuris. He II Heat Transfer Through Superconducting Cables Electrical Insulation. Cryogenics, 40:127 $136,2000$.

[47] C. Meuris, B. Baudouy, D. Leroy, and B. Szeless. Heat Transfer in Electrical Insulation of LHC Cables Cooled with Superfluid Helium. Cryogenics, 39:921 $-931,1999$.

[48] N. Kimura, A. Yamamoto, T. Shintomi, and A. Terashima. Heat Transfer Characteristics of Rutherford-Type Superconducting Cables in Pressurized He II. IEEE Transactions on Applied Superconductivity, 9(2):1097 - 1100, 1999.

[49] M. La China and D. Tommasini. Cable Insulation Scheme to Improve Heat Transfer to Superfluid Helium in Nb-Ti Accelerator Magnets. IEEE Transactions on Applied Superconductivity, 18(2):1285 - 1288, 2008.

[50] M. La China and D. Tommasini. Comparative Study of Heat Transfer from Nb-Ti and $\mathrm{Nb}_{3}$ Sn Coils to He II. Phys. Rev. ST Accel. Beams, 11:082401, Aug 2008.

[51] P.P. Granieri, P. Fessia, D. Richter, and D. Tommasini. Heat Transfer in an Enhanced Cable Insulation Scheme for the Superconducting Magnets of the LHC Luminosity Upgrade. IEEE Transactions on Applied Superconductivity, 20(3):168 - 171, June 2010.

[52] P.P. Granieri. Heat Transfer Between the Superconducting Cables of the LHC Accelerator Magnets and the Superfluid Helium Bath. PhD thesis, EPFL, 2012.

[53] P.P. Granieri. Heat Transfer Through Cable Insulation of NbTi Superconducting Magnets Operating in He II. Cryogenics, 53:61 - 71, 2013.

[54] P.P. Granieri, D. Santandrea, T. Koettig, and R. van Weelderen. Heat Extraction from the LHC Main Dipole, Main Quadropole and MQXA Superconducting Cables. IEEE Transactions on Applied Superconductivity, 25(3), June 2015.

[55] M.N. Wilson. NbTi Superconductors with Low AC Loss: A Review. Cryogenics, 48:381 - 395, 2008.

[56] Cernox thin film resistance thermometer. https://goo.gl/olN5Iy, 102016.

[57] A.M Campbell. A General Treatment of Losses in Multifilamentary Superconductors. Cryogenics, 22:3 - 16, 1982.

[58] Y. Iwasa. Case Studies in Superconducting Magnets. Kluwer Academic Publishers, 2002. 
[59] A. Verweij. Electrodynamics of Superconducting Cables in Accelerator Magnets. PhD thesis, University of Twente, 1995.

[60] D. Richter, J.D. Adam, J.-M. Depond, D. Leroy, and L.R. Oberli. DC Measurements of Electrical Contacts between Strands in Superconducting Cables for the LHC Main Magnets. IEEE Transactions on Applied Superconductivity, $7(2), 1997$.

[61] MKS 651C Digital/Analog Pressure Controller. https://goo.gl/EnTtTb, 10 2016.

[62] MKS 626C Baratron Absolute Capacitance Manometer. https://goo.gl/eGfHYm, 102016.

[63] MKS 653B Exhaust Throttle Valve. https://goo.gl/WmhPxP, 102016.

[64] NI USB-6225. https://goo.gl/Fvs4vH, 102016.

[65] Keithely 2400 SourceMeter SMU Instruments. https://goo.gl/FEU2s6, 10 2016.

[66] Keithley Series 3700A Systems Switch/Multimeter. https://goo.gl/8xXMJw, 102016.

[67] E.W. Collings, R.D. Smith, J.C. Ho, and C.Y. Wu. Low Temperature Specific Heat and Magnetic Susceptibility of NbTi and NbTiMn Alloys. Advances in Cryogenic Engineering (Materials), 36:239 - 246, 1990.

[68] F. Pobell. Matter and Methods at Low Temperatures. Springer-Verlag Berlin Heidelberg, 2007.

[69] W.M. van Alphen, G.J. van Haasteren, R. de Bruyn Ouboter, and K.W. Taconis. The Dependence of the Critical Velocity of the Superfluid on Channel Diameter and Film Thickness. Physics Letters, 20(5):474 - 475, 1966.

[70] K.P. Martin and J.T. Tough. Evolution of Superfluid Turbulence in Thermal Counterflow. Physical Review B, 27(5):2788 - 2799, March 1983.

[71] J.D. Adam, T. Boutboul, G. Cavallari, Z. Charifoulline, C.-H. Denarie, S. Le Naour, D.F. Leroy, L. Oberli, D. Richter, A.P. Verweij, and R. Wolf. Status of the LHC Superconducting Cable Mass Production. IEEE Transactions on Applied Superconductivity, 12(1), 2002.

[72] B. Baudouy. Kapitza Resistance and Thermal Conductivity of Kapton in Superfluid Helium. Cryogenics, 43(12):667 - 672, 2003.

[73] Cryodata Inc. HEPAK(C) software package.

[74] S.W. van Sciver. Transient Heat Transport in He II. Cryogenics, 19(7):385 392, July 1979 
[75] W.F. Vinen. Mutual Friction in a Heat Current in Liquid Helium II. II. Experiments on Transient Effects. Proceedings of the Royal Society of London, Series A, Mathematical and Physical Sciences, 240(1220):128 - 143, April 1957. 


\section{Summary}

The European Organization for Nuclear Research (CERN) in Geneva is operating the currently most powerful particle accelerator, the Large Hadron Collider (LHC). This particle accelerator is a $27 \mathrm{~km}$ long ring and it uses very strong electro magnets to contain the particles on the circular path. The wire used for the electromagnets is made from the superconductor Niobium-Titanium, a material that needs to be cooled with liquid helium in order to be superconducting. A superconductor needs to be keep below its critical temperature in order to maintain the superconducting properties, in the LHC this is achieved by a He bath at $1.9 \mathrm{~K}$ in which the superconductor is placed. Helium at this temperature is referred to as He II because of its very different fluid properties compared to helium at temperatures above $2.176 \mathrm{~K}$.

During the operation of the LHC heat loads are generated due to different processes such as beam collimation, synchrotron radiation or residual gas scattering and the fluid properties of He II are beneficial to the cooling of the magnet due to its high effective thermal conductivity. The heat loads are generated on different time scales and either occur continuously or only occur for a limited duration. Therefore the heat transfer between the superconducting cable and the cooling $\mathrm{He}$ bath needs to be known as a function of time in order to guarantee a safe operation of the LHC.

Thermal stability of the superconducting magnets of the LHC so far has been measured on different geometric levels. Measurements have been conducted on a single strand level, the level of a single Rutherford cable with different insulation types and lastly also with stacks of Rutherford cables to simulate the magnet geometry. In the experiments the sample is heated either locally or distributed to investigate the thermal stability of the superconducting cable.

In this work the heat transfer through the polyimide cable insulation between the superconducting cable and the He bath is studied in steady-state as well as in transient state with a stack of superconducting cables in case of distributed loads. In order to investigate the heat transfer a new set-up was developed that uses $\mathrm{AC}$ loss to generate heat in a superconducting cable. In this way a sample could be taken from a production grade batch of superconducting cable with a polyimide insulation applied by a machine. This is in stark contrast to previous experiments that used a non-superconducting cable with a hand-wound polyimide insulation to investigate the heat transfer.

In this study a stack of four superconducting cables is investigated and the temporal evolution of the cable temperature is recorded by two Cernox temperature sensors that are placed carefully in one of the centre two cables of the stack. Different experiments are conducted, where the He bath temperature, the saturation conditions of the He bath, the mechanical pressure on the sample and the heat 


\section{Summary}

load are varied. The He bath temperature is varied between $1.7 \mathrm{~K}$ to $2.1 \mathrm{~K}$ with a focus on $1.9 \mathrm{~K}$, the operation temperature of the LHC in a saturated He bath as well as in a pressurised He bath at 1.2 bar. The heat load is varied between $0.61 \mathrm{~mW} \mathrm{~cm}^{-3}$ to $4.9 \mathrm{~mW} \mathrm{~cm}^{-3}$ and the mechanical pressure on the sample is set to either $50 \mathrm{MPa}$ or $75 \mathrm{MPa}$.

It was found that a single exponential function describes the temporal evolution of the sample temperature very well and two values can be extracted from the exponential fit function, the steady-state temperature difference and the characteristic time constant.

From the steady-state temperature difference it was found that besides the solid conduction path through the insulation at least two additional heat transfer paths are linking the cable to the He bath. Both heat transfer paths are created by small channels through the polyimide insulation and are filled with He II. The two channels can be distinguished by the characteristic behaviour of heat transfer in He II depending on the heat transfer regime. He II has two very different heat transfer regimes, a laminar regime and a turbulent regime, the laminar regime has a linear dependency between the transported heat load and the temperature difference, whereas the turbulent regime has an approximately cubic dependency. Both dependencies are seen in the measurement results and the modelling results show that the turbulent heat transfer path is dominant for low heat loads and with increasing heat load the laminar heat transfer path is becoming dominant. Therefore it was concluded that at least two independent heat transfer paths connect the sample and the He bath.

The steady-state temperature difference is simulated with a model consisting of two independent helium channels with different geometries besides the solid conduction through the polyimide insulation. From the modelling two geometry coefficients are obtained. The geometry coefficients are compared for different mechanical conditions and the suitability of the model in a wide temperature range is shown, indicating that the model is suitable to describe the thermal link between a heated superconducting Rutherford cable with a polyimide insulation as used in the LHC and a cooling He bath.

From the time constant it can be seen that during the transient phase of the experiment the turbulent heat transfer path is strongly affected by the temperature gradient at low heat loads and as a result the turbulent effective thermal conductivity is reduced. For higher heat loads the turbulent effective thermal conductivity is still affected by the temperature gradient but its effect is less severe. This leads to the conclusion that the laminar heat transfer channel is very important in the transient phase of the experiment.

This work shows results for the heat transfer between a superconducting Rutherford cable and a He bath with a machine made polyimide insulation. This machine made insulation allows to characterise the heat transfer better than with a handwound insulation as it is used so far. The transient heat transfer is studied and the effect of the temperature gradient on the effective thermal conductivity is identified as the determining factor for the time constant. 


\section{Samenvatting}

De Europese Organisatie voor Nucleair Onderzoek (CERN) in Geneve heeft momenteel de meest krachtige deeltjesversneller, de Large Hadron Collider (LHC) in gebruik. Deze deeltjesversneller bestaat uit een $27 \mathrm{~km}$ lange ring waarbij sterke elektromagneten ervoor zorgen dat de versnellende deeltjes in hun cirkelvormige baan blijven. Voor deze magneten wordt gebruik gemaakt van supergeleidend Niobium-Tin draad, een materiaal dat gekoeld moet worden met vloeibaar helium om de supergeleidende toestand te bereiken, wat bereikt wordt wanneer de geleider gekoeld wordt tot beneden zijn kritieke temperatuur. In de LHC worden de magneten gekoeld met behulp van vloeibaar helium op $1.9 \mathrm{~K}$. Op deze temperatuur staat helium ook bekend als HeII (helium 2), aangezien HeII andere eigenschappen heeft vergeleken met normaal vloeibaar helium boven een temperatuur van ruwweg $2.2 \mathrm{~K}$. Als de LHC in werking is, is er sprake van warmtebelastingen door verschillende processen, waaronder synchrotronstraling, beam collimation of gas verstrooiing. Het blijkt dat de hoge thermische geleidbaarheid van HeII essentieel is voor het koelen van de magneten. De genoemde warmtebelastingen worden gegeneerd op verschillende tijdsschalen en kunnen continu of op een onregelmatige manier voorkomen. Om hier goed mee om te gaan moet de warmteoverdracht tussen de supergeleidende kabel en het helium-bad bekend zijn als functie van de tijd om zo een veilige operatie van de LHC te garanderen. De thermische stabiliteit van supergeleidende magneten van de LHC is tot dusver gemeten op verschillende grootte schalen: van draad-niveau tot metingen op een enkele Rutherford kabel met verschillende isolaties. Ook zijn er metingen gedaan op een bundel van Rutherford kabels, dit om de uiteindelijke geometrie van de magneet beter te simuleren. Tijdens deze metingen werd gecontroleerd warmte toegevoegd op de proefstukken, zowel lokaal alswel gelijkmatig verspreid, om de thermische stabiliteit van de supergeleidende kabel tot in detail te kunnen onderzoeken. In dit proefschrift wordt de warmteoverdracht tussen de isolatie van de supergeleidende kabel en het helium-bad beschouwd onder statische en dynamische condities met een stapeling van supergeleidende kabels en uniform verdeelde warmtepulsen. Hiervoor is een nieuwe opstelling ontwikkeld welke gebruik maakt van $\mathrm{AC}$ verliezen om warmte in de kabel te genereren. Op deze manier kon gemeten worden aan een proefstuk van de supergeleidende kabel dat rechtstreeks uit het productieproces genomen kon worden. Dit is in tegenstelling tot eerdere experimenten waarbij het proefstuk bestond uit een niet-supergeleidende kabel en de isolatie met de hand was aangebracht. In deze studie is gekeken naar een bundel van vier supergeleidende kabels. Het verloop van de temperatuur in de tijd is geregistreerd met twee Cernox temperatuursensoren die nauwkeurig in het centrum van de bundel waren geplaatst. Verschillende experimenten zijn uitgevoerd, waarbij de temperatuur van het helium-bad, de verzadigingscondities van het helium-bad, de mechanische druk 
op het proefstuk en de warmte-invoer gevarieerd konden worden. Het helium-bad had een temperatuur welke was ingesteld tussen de $1.78 \mathrm{~K}$ en $2.1 \mathrm{~K}$ met specifiek aandacht voor $1.9 \mathrm{~K}$, de temperatuur waarop de LHC werkt in een situatie met verzadigd $\mathrm{He}$ of He op 1.2 bar. De warmtebelasting werd gevarieerd tussen de $0.61 \mathrm{~mW} \mathrm{~cm}^{-3}$ en $4.9 \mathrm{~mW} \mathrm{~cm}^{-3}$ helium-bad en de druk op de kabels was $50 \mathrm{MPa}$ of $75 \mathrm{MPa}$. Gebleken is dat een enkele exponentile functie het verloop van de temperatuur in de tijd goed beschrijft en er twee waarden uit deze exponentile fit gehaald kunnen worden; het statische temperatuurverschil en de karakteristieke tijdsconstante. Met het statische temperatuurverschil werd duidelijk dat naast een vast thermisch geleidingspad door de isolatie er tenminste twee warmtepaden zijn die de kabels verbinden met het helium-bad. Beide paden worden gecreerd door kleine kanalen die door de isolatie heen lopen. Deze kanalen zijn gevuld met HeII en worden gekenmerkt door het karakteristieke gedrag van de warmteoverdracht van HeII, afhankelijk van het regime waarin de vloeistof zich bevindt. HeII heeft namelijk twee verschillende warmte-regimes; laminair en turbulent. Het laminaire regime kenmerkt zich door een lineair verband tussen de warmteoverdracht en het temperatuurverschil, terwijl voor het turbulente regime een bijna kubische afhankelijkheid geldt. Beide afhankelijkheden zijn terug te vinden in de gepresenteerde metingen. Daarnaast laten de gemodelleerde resultaten zien dat het turbulente pad dominant is bij lage warmtebelastingen. Naarmate de belasting wordt verhoogd zal het laminaire deel een grotere rol spelen. Hieruit is te concluderen dat er tenminste twee onafhankelijke warmtepaden zijn tussen het proefstuk en het helium-bad. Het temperatuurverschil in de statische situatie is gesimuleerd met behulp van een model bestaande uit twee onafhankelijke He kanalen met verschillende geometrien naast het vaste geleidingspad door de isolatie. Uit deze modellen konden twee geometrische cofficinten gehaald worden. Deze zijn vergeleken met verschillende mechanische condities. Bovendien is de toepasbaarheid van het model in een groot temperatuurbereik aangetoond. Dit laat zien dat het model geschikt is om de thermische verbinding tussen een verwarmde supergeleidende Rutherford kabel, inclusief de isolatie zoals gebruikt wordt in de LHC, en het helium-koelingsbad te beschrijven. Door naar de tijdconstante te kijken werd duidelijk dat in de dynamische fase van het experiment, het turbulente He pad sterk benvloed werd door de thermische gradint bij lage warmtebelastingen. Als gevolg hiervan is de effectieve thermische geleidbaarheid lager. Bij hogere belastingen is de geleidbaarheid nog steeds lager, maar is het effect ten gevolge van de thermische gradint nu minder sterk aanwezig. Hieruit is te concluderen dat de laminaire warmteoverdracht vooral belangrijk is in de dynamische fase van de opwarming. Dit werk presenteert de resultaten voor de warmteoverdracht tussen een Rutherford kabel, waarbij de isolatie met een machine is aangebracht, en een helium-bad. Doordat de isolatie niet met de hand is uitgevoerd, is de warmteoverdracht beter te beschrijven dan tot dusver is gedaan. De warmteoverdracht is bestudeerd in verschillende fases van het experiment en het effect van de thermische gradint op de thermische geleidbaarheid bleek de bepalende factor te zijn voor de tijdsconstante. 


\section{Acknowledgement}

Writing a $\mathrm{PhD}$ is a journey through the academic world. A journey that leads through a world full of challenges and obstacles. But the same world also holds the rare reward and feeling of success ready for just the right moment when nothing works and the frustration is about to get a permanent hold of you.

During this adventure I met a lot of interesting people and I would like to thank some of them for their support and help.

Firstly I would like to thank my supervisor Marcel ter Brake for agreeing to accompany me on my adventure after a phone call and a personal discussion. My thanks also extend to Rob van Weelderen as my supervisor at CERN and Torsten Koettig for his day-to-day supervision. All three of you have helped me with extensive discussions to move on, increased the value of my work with critical comments and finally have stood by my side when things were difficult.

After the completion of the practical work of a $\mathrm{PhD}$ comes the part where different people known in their research field are asked to evaluate the work. I like to thank all members of my PhD committee, Betrand Baudouy, Marco Breschi, Marc Dhalle, Bob van Eijk, Herman ten Kate and Fons de Waele for agreeing to judge my work.

An experimental $\mathrm{PhD}$ thesis is not possible without experiments. And when it comes to realising an experiment there is whole group of people whose precious advise and help made the experiments possible. Two people of this group I would like to mention first: Johan Bremer, the head of the Cryolab \& Instrumentation Section at CERN who had my back and the political connections to make things happen and Laetitia Dufay-Chanat, who had an open ear and a helping hand whenever it was needed. Also all technicians from the Cryolab need to be mentioned here: Jean-Luc Carpano, Daniel Cochet, Florian Girardot, Sergio Guido, Sebastien Prunet, Jean-Louis Servais, Tino Vacca. I thank you all for accepting me in your circle, for your helping hand and for having the patience to teach and explain the French language to me in numerous coffee breaks.

Working for four years on a very specific topic is impossible without colleagues who get you back on solid ground when things get too far off.

I spend three years at CERN and during that time I met Waldemar Maciocha and Joanna Liberadzka. I thank you two for our discussions about everything and nothing, the funny moments and most importantly for providing the occasionally needed motivational boost.

During my time as a $\mathrm{PhD}$ student I had the pleasure to work with different students. I would like to thank Bruno Martins Galinhas, Alexandra Onufrena and Jens Schundelmeier for their help in preparing the sample, the set-up and in conducting the experiments. Bruno also deserves to be mentioned here for his work on the heat transfer model. 
The last year of my PhD I spend at the EMS group of the University of Twente. I would like to thank all of you for giving me such a warm welcome. Ans Veenstra definitely needs to be mentioned here for organising everything nicely, knowing the answers to all those questions and for making me feel welcome. EMS, you are a great working group and it was a pleasure to work with you. Two people I would like to pick out from the crowd: Anne, Chris, thank you very much for listening and cheering me up during the last year of my PhD. The coffee breaks would only have been half as much fun without you.

During my time in Enschede I met interesting people in the table tennis association as well as in the archery association. It was a pleasure to play table tennis with you and to shoot next to you.

Most of the time during my PhD I was talking English, then it is always a pleasant surprise to meet fellow country(wo)men und die Möglichkeit zu haben einfach so die Sprache zu wechseln. Die Kartenspielrunde am CERN war immer eine willkommene Ablenkung von der Arbeit, dafür bin ich euch dankbar und ich denke gerne an die gemeinsamen Abende zurück. Clara, an der UTwente hast du diese Position übernommen. Mein Englisch mag gut sein, aber Deutsch ist nun einmal meine Muttersprache. Es war mir eine Freude mich mit dir zu unterhalten und sich gegenseitig aufzuziehen.

Minke, talking with you was one of the most comforting things during my PhD.

4 years is a long time and I am grateful for my friends that accompanied me along the journey and friends that I met during this time.

Mariann, ich danke dir für deine Unterstützung und deine Geduld mit mir, unsere Telefonate und Treffen sind ein wichtiger Teil meines Weges. Wie sehen uns leider viel zu selten, umso mehr freue ich mich jedes Mal wenn wir uns treffen.

Miri, es freut mich jedes Mal aufs Neues wenn wir uns treffen. Und jedes Mal ist es so als hätten wir uns erst letzte Woche gesehen oder gesprochen.

Bene, die Nm!-Bar ist einer der geselligsten Orte die ich kenne und die Abende die wir dort verbracht haben werden mir noch lange in Erinnerung bleiben.

Micha, es war immer eine Freude mit dir zu philosophieren über alles was uns gerade einfiel. Ich danke dir für all die Scherze, die das Leben und das Promovieren im besonderen angenehmer gemacht haben.

Tommaso, our discussions on-topic and mostly off-topic are a constant source of laughter. Your opinion from the outside is always precious when I thought I was lost somewhere and had no idea how to explain myself.

Und zum Schluss möchte ich mich bei meiner Familie bedanken. Ihr habt mir wenn nötig mit Rat und Tat aber immer mit moralischer Unterstützung diesen Weg erleichtert.

As far as the nature of an acknowledgement goes this is not an exclusive list. I would like to thank all those people who I forgot to mention and who deserve to be thanked. 